\title{
IMPROVED APPROXIMATE: FORMULAS FOR FLUX FROM CYLINDRICAL AND RECTANGULAR SOURCES
}

O. J. Wallace

is. A. Bokiharee

Contract No. DE-AC11-88PN38014

March 1993

\author{
Printed in the United States of America \\ Avallable from the \\ National Techrical Iriformation Service \\ U. S. Deplartment of Conmerce \\ 5285 Port Royal Road \\ Springfield, Virginia 22161
}

NOTE

This document is an interim memorandum prepareit primarily for internal reference and does not represent a final expression of the opinion of Westirighouse. When this memorandum is distributed externally, it is with the express understanding that Westinghouse makes no representation as to completeness, accuracy, or usability of information sontained herein. 


\section{DISCLAIMER}

This report was prepared as an account of work sponsored by an agency of the United States Government. Neither the United States Government nor any agency Thereof, nor any of their employees, makes any warranty, express or implied, or assumes any legal liability or responsibility for the accuracy, completeness, or usefulness of any information, apparatus, product, or process disclosed, or represents that its use would not infringe privately owned rights. Reference herein to any specific commercial product, process, or service by trade name, trademark, manufacturer, or otherwise does not necessarily constitute or imply its endorsement, recommendation, or favoring by the United States Government or any agency thereof. The views and opinions of authors expressed herein do not necessarily state or reflect those of the United States Government or any agency thereof. 


\section{DISCLAIMER}

Portions of this document may be illegible in electronic image products. Images are produced from the best available original document. 


\section{NOTICE}

This report was prepared as an account of work sponsored by the United States Government. Neither the! United States, nor the United States Department of Energy, nor any of their employees, makes any warranty, expressed or implied, or assumes any legal liability or responsibility for the accuracy, completenes:s or usefulness of any information, apparatus, produce or process disclosed, or represents that its use would not infringe privately owned right:s. 
Improved Analytic Flux Formulas for Cylindrical and Rectangular Sources

\section{TABLE OF CONTENTS}

Page

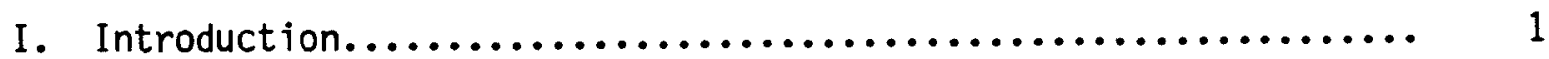

II. Background - The Approximation of Ono and Tsuro.............. 2

A. An Exact Flux Formula for a Cylindrical Source............ 2

B. The Approximation of Ono and Tsuro.................... 5

C. 1. The Functions of Ono and Tsuro.................... 7

C. 2. A Sample Problem................................ 8

D. Improved Accuracy for the Approximation of Ono and Tsuro.... 9

III. Extensions of the Approximation of Ono and Tsuro - Approximate Formulas for the Flux at Detector Points Outside the Radial and Axial Extensions of a Cylindrical Source with a Slab Shield.............................................

A. Background....................................... 9

B. CASE I - Slab Shield Parallel to the Source Axis............ 10

C. CASE II - Slab Shield Perpendicular to the Source Axis...... 13

IV. Approximations of Rectangular Sources.................... 16

A. Approximation of a Rectangular Volume Source by a Section of an Annulus................................ 16

B. Approximation of Rectangular Source with Square Cross-Section by a Truncated Cone........................ 17

C. Approximation of a Rectangular Surface Source.............. 18

D. Rectangular Surface Source Sample Problems............... 20

1. Square Surface Source Problem.................... 20

2. Rectangular Surface Source Problem................. 23

v. Figures......................................... 25

1 Division of a Cylindrical Source into Regions I and II..... 25

2 Detail of Division of a Cylindrical Source into Regions I and II...................................... 


\section{TABLE OF CONTENTS (Cont'd)}

Page

3 Cylindrical Source-Volume: Approximation Using a Section of an Annulus with a Curved Top Surface............ 26

4 Bases of Cylinder and Annular Sector.................. 27

5 Cylindrical Source Approximation with Detector Point Outside the Axial and Radial Extensions of the Source and with a Slab Shield Parallel to the Source Axis......... 28

6 Detail of the Midplane of a Cylindrical Source Approximation with Detector Point Outside the Axial and Radial Extensions of the Source................................

7 Layout of Arbitrary Test Problem with Slab Shield Parallel

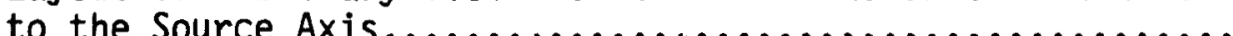

8 Cylindrical Source with a Detector Point Outside the Radial and Axial Extensions of the Source and with a Slab Shield Perpendicular to the Source Axis...............

9 Section of an Annulus Used to Approximate a Cylindrical Source with a Detector Point Outside the Radial and Axial Extensions of the Sclurce, and with a Slab Shield

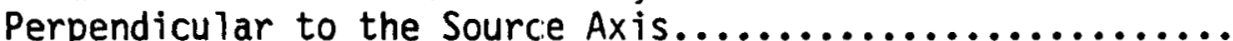

10 Layout of Arbitrary Test Froblem with a Slab Shield

Perpendicular to the Source Axis....................... 31

11 Base of an Annular Sector which Preserves the Area of the Base of a Rectangular Source.................... 32

12 Rectangular Volume Source Approximation Using a Section of an Annulus............................... 33

13 Square Rectangular Source Approximation Using a

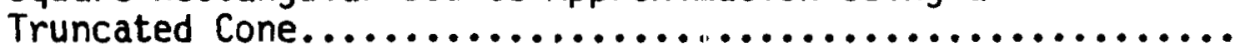

14 Rectangular Surface Source with Center Opposite Detector Point....................................... 
TABLE OF CONTENTS (Cont'd)

VI. Tables....................................................

1 Approximate Flux for a Cyindrical Source with a

Lateral Detector Point and a Slab Shield Parallel to the Source Axis........................................

1 A Improved Ratios of Approximate Flux Using the Algorithm of Section II.D.

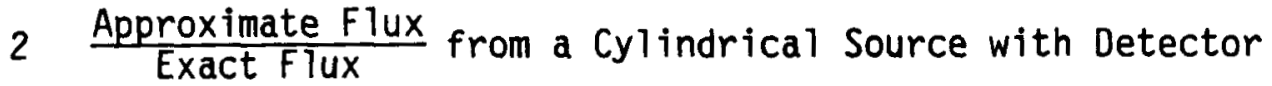

Points Outside the Radial and Axial Extensions of the Source with an Intervening Slab Shield Parallel to the Source Axis.

3 Results from the Exact Flux Formula and from Equation

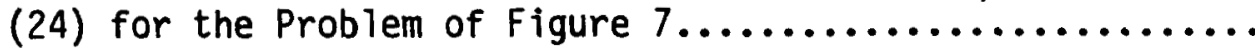

4 Approximate Flux from a Cylindrical Source at Detector Points Outside the Radial and Axial Extensions of the Source with an Intervening Slab Shield Perpendicular to the Source Axis.....................................

5 Results from the Exact Flux Formula and From Equation (30)

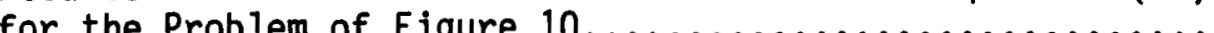

6 The Function $L_{0}\left(\phi_{0}, b\right) \ldots \ldots \ldots \ldots \ldots \ldots \ldots \ldots \ldots \ldots \ldots \ldots \ldots$

7 The Function $\left(\theta_{0}, b\right) \ldots \ldots \ldots \ldots \ldots \ldots \ldots \ldots \ldots \ldots \ldots \ldots, 45$

8 The Smoothed Function $\bar{G}\left(\theta_{0}, b\right)=G\left(\theta_{0}, b\right) e^{b} / \theta_{0} \ldots \ldots \ldots \ldots .45$

9 The Smoothed Function $\bar{F}\left(\theta_{0}, b\right)=F\left(\theta_{0}, b\right) e^{b} / \theta_{0} \ldots \ldots \ldots \ldots .46$

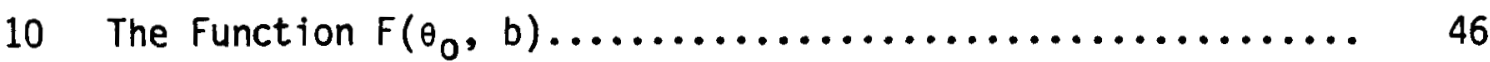

11 The Smoothed Function $\bar{F}_{2}\left(\theta_{0}, b\right)=F_{2}\left(\theta_{0}, b\right) e^{b} / \theta_{0} \ldots \ldots \ldots . .47$

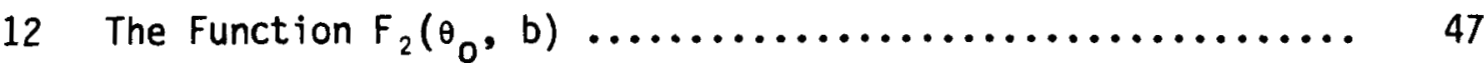




\section{TABLE OF CONTENITS (Cont'd)}

13 The Smoothed Function $\bar{R}\left(\phi_{0}, l_{2} / a, b\right)=R\left(\phi_{0}, l_{2} / a, b\right) e^{b} / \phi_{0} \frac{\frac{\text { Page }}{48}}{48}$

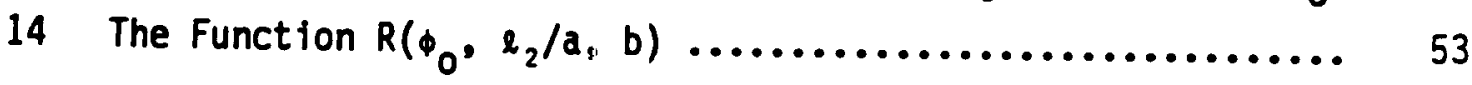

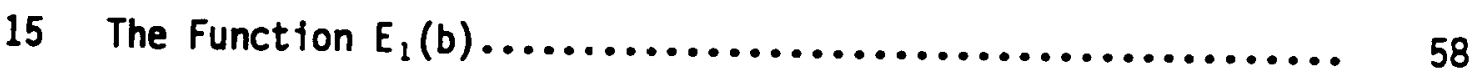

16 The Smoothed Function $\bar{E}_{1}(b)=b e^{b} E_{1}(b) \ldots \ldots \ldots \ldots \ldots \ldots . . \ldots$

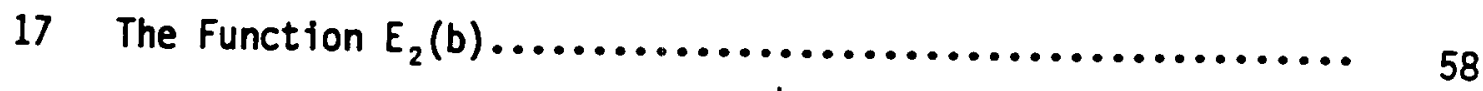
18 The Smoothed Function $\bar{E}_{2}(b)=b_{1}{ }^{b} E_{2}(b) \ldots \ldots \ldots \ldots \ldots \ldots . . \ldots \ldots$ VII. References.......................................... 59 


\section{ABSTRACT}

\section{IMPROVED APPROXIMATE FORMULAS FOR FLUX} FROM CYLINDRICAL AND RECTANGULAR SOURCES

This report provides two new approximate formulas for the flux at detector points outside the radial and axial extensions of a homogeneous cylindrical source and improved approximate formulas for the flux at points opposite rectangular surface sources. These formulas extend the range of geometries for which analytic approximations may be used by shield design engineers to make rapid scoping studies and check more extensive calculations for reasonableness. These formulas can be used to support skeptical, independent evaluations and are also valuable teaching tools for introducing shield designers to complex shield analyses. 


\section{Introduction}

Hand calculation methods involving analytic approximations of exact flux formulas and tables of special mathenlatical functions continue to be useful in shielding calculations since they enable shield design personnel to make quick estimates of dose rates, check calculations made by more exact and time-consuming methods, and rapidly determine the scope of problems before a detailed solution is attempted.

They are also a valuable teaching tool. Solving shielding problems by hand methods can give a shielding engineer a body of experience and a feel for such problems which is very valuable when the output from black-box type computer programs must be evaluated.

Knowledge of hand calculation methods of radiation fluxes and dose rates is also valuable and even essential in those situations where no computer, or no time to use a computer, is available, and a quick answer is needed.

Several collections of approximate analytic radiation flux formulas are available, as are tables of the required mathematical functions. A partial list of these documents is given in Section $H$ of Reference (1). Reference (1) is itself a compilation of useful approximate analytic flux calculation formulas and function tables which were assembled expressly for the use of shieliding pelsonnel. Reference (2) contains a subset of the same material. Reference (1) gives approximate flux formulas for a variety of source geometries and contains tables of cross-section, spectrum, and build-up factor data as well as shielding function tables and considerable information about these functions. This report assumes that the reader is at least minimally familiar with the contents of Reference (1).

Extensive use of Reference (1) has shown that improved analytic approximate formulas are needed for finding the flux

(a) at detector points located outside the radial and axial extent of a cylindrical source, and

(b) at detector points opposite a rectangular source.

In all cases, the attenuation of a slab shield must be included. 
In addition, more extensive information on the errors in results calculated using the cylindrical source approximation of Ono and Tsuro [References (1)-(4)] is needed, and improvement in this formula is desirable.

The material given in later sections of this report meets all these needs. Therefore this report supplements and extends the formulas and information given in Reference (1), and the two reports should be mutually familiar to the user. The use of this material should enable shielding engineers to make better estimates and quick scoping calculations in many situations and thereby save engineering and computer time.

In order that the reader may better understand the approximate flux formulas given in this report, Section II below starts with the exact formula for flux from a cylindrical source at a lateral detector point and then discusses the corresponding cylindrical source approximation of Ono and Tsuro [Reference (4)] in detail, ending with a refinement which improves its accuracy in some cases.

Section III then presents extensions of the cylindrical source approximate flux formula which apply to detector points located outside the radial and axial extent of a cylindrical source. Detailed error information is given for all approximate cylindrical source formulas.

Finally, Section IV discusses calculation of flux from rectangular sources and gives both an exact formula and a new approximation for a rectangular surface source.

All required mathematical function tables are given in Section VI.

II. Background - The Approximation of Ono and Tsuro

A. An Exact Flux Formula for a Cylindrical Source

Figures 1 and 2 show a cylindrical source with a lateral detector point. A slab shield of thickness $t$ is assumed to be parallel to the source axis, perpendicular to the line $O P$, and to lie between the source and the detector point $P$.

The unscattered flux at the point detector $p$ is given by

$$
\phi_{p}=\int_{V_{s}} \frac{S_{v} e^{\left[-\mu_{s}\left(r_{2}-r_{1}\right)+\mu t\right] \sec \phi \sec \theta}}{4 \pi \rho^{2}} d V_{s}
$$


where

$S_{v}=$ Source strength pier unit volume per unit time

$u_{s}=$ Attenuation coefficient of the source material

$\mu=$ Attenuation coefficient of the shield material

$t=$ Thickness of the shield

$d V_{s}=$ Volume element of the source

$r_{2}-r_{1}=$ Distance from the volume element to the cylindrical source surface in the direction of the detector, projected onto the base of the cylinder. See Figures 1 and 2.

$\phi=$ Angle subtended by the cylinder, as seen from the detector point. See Figures 1 and 2.

$\theta=$ Angle between the straight line connecting the volume element with the point detector and a straight line passing through the point detector and normal to the shield. $\theta$ is a function of the height of the cylinder. See Figures 1 and 2.

$\rho=$ Distance from the volume: element to the point detector.

The integral can be simplified by choosing the Ono and Tsuro method which regards the point detector as the origin of the coordinate system (See Reference (4)). Then from Figures 1 and 2 it can be ascertained that

$$
\rho=r \sec \phi \sec \theta
$$

$$
\begin{aligned}
r_{1} & =(R+a) \cos ^{2} \phi-\cos \phi \sqrt{R^{2}-(R+a)} \overline{2 \sin ^{2} \phi} \\
r_{2} & =(R+a) \cos ^{2} \phi+\cos \phi \sqrt{R^{2}-(R+a)} \overline{\sin ^{2} \phi} \\
r_{2}-r_{1} & =2 \cos \phi \sqrt{R^{2}-(R+a)^{2}} \overline{\sin ^{2} \phi} \\
d v_{s} & =d r \cdot d_{\phi}(r \tan \phi) \cdot d z \\
& =d r \cdot d_{\phi}(r \tan \phi) \cdot d_{\theta}\left(r \cdot \sec _{\phi} \tan \theta\right)
\end{aligned}
$$

where subscripts indicate the variation in that direction. 


$$
\begin{aligned}
\therefore \mathrm{dV}_{s} & =d r \cdot\left(\frac{\partial}{\partial \phi}(r \tan \phi) \cdot d \phi\right) \cdot\left(\frac{\partial}{\partial \theta}(r \sec \phi \tan \theta) \cdot d \theta\right) \\
& =d r \cdot\left(r \sec ^{2} \phi d \phi\right) \cdot\left(r \sec \phi \sec ^{2} \theta d \theta\right) \\
d V_{s} & =r^{2} \sec ^{3} \phi \sec ^{2} \theta d r d \phi d \theta
\end{aligned}
$$

The angles are given by

$$
\begin{aligned}
& \phi_{0}=\sin ^{-1}(R /(a+R)) \\
& \theta_{3}=\tan ^{-1} \ell / r_{2} \sec \phi \\
& \theta_{4}=\tan ^{-1} \ell / r_{1} \sec \phi
\end{aligned}
$$

as may be seen from Figures 1 and 2 .

By combining equations (1), (2a) and (2e) we may write

$$
\begin{array}{r}
\phi_{p}=\frac{S_{v}}{4 \pi \mu_{s}} \cdot 2 \int_{0}^{\phi_{0}} \sec \phi d \phi \int_{\mu_{s} r_{1}}^{\mu_{s} r_{2}} d(\mu r) \int_{s}^{\theta_{0}} \exp (-\mu t \sec \phi \sec \theta) \cdot \\
\quad \exp \left(-\mu_{s}\left(r-r_{1}\right) \sec \phi \sec \theta\right) \cdot d \theta
\end{array}
$$

The integration over $r$ can easily be carried out with the result

$$
\begin{aligned}
& \phi=\frac{S_{v}}{2 \pi \mu_{S}} \int_{0}^{\phi} g_{\phi} \int_{0}^{\theta} \cos \theta \cdot \exp (-b \sec \theta \sec \phi) \cdot\left[1-\exp \left(-\mu_{S}\left(r_{2}-r_{1}\right)\right) \sec \theta \sec \phi \cdot\right] d \theta \\
& \text { where } b=\mu t . \\
& \text { Let } G\left(\theta_{0}, \mu t\right)=\int_{0}^{\theta} \cos \theta \cdot \exp (-\mu t \sec \theta) d \theta \\
& \text { Then Equations (2d), (3) and (4) yield }
\end{aligned}
$$




$$
\begin{aligned}
& \phi=\frac{1}{2 \pi \mu_{s}} \int_{0}^{\phi_{0}}\left[G\left(\theta_{0}, \mu t \sec \phi\right)-G\left(\theta_{0},\left(\mu t \sec \phi+2 \mu_{s} R \overline{\left.1-(1+a / R)^{2} \sin ^{2} \phi\right)}\right] d \phi\right.\right. \\
& \quad G\left(\frac{\pi}{2}, \mu t\right)=H(\mu t)=\int_{0}^{\frac{\pi}{2}} \cos \theta \operatorname{sexp}(-\mu t \sec \theta) d \theta
\end{aligned}
$$

B. The Approximation of Ono and Tsuro

Equation (1) must be solved by numeric integration or quadrature if it is to be solved at all. Ono and Tsuro were able to derive an ingenious and useful approximation to Equation (1) by substituting a section of an annulus for the cylinder, as shown in Figure 3. To quote from their paper:

"A cylindrical source is replaced by a columnar fragment of cylindrical shell with area of base equal to that of the original cylinder. (See Fig. 3). The cylindrical shell fragment has six surfaces, two plane side surfaces, a conical top surface, and a bottom surface. Consider a straight line $L$ parallel to the axis of the source cylinder and passing the observation point $P$. The inner surface is part of a cylinder and passing the observation point $P$. The inner surface is part of a cylinder with axis coinciding with the line $L$ and radius equal to $a$. The two side surfaces contain the 1 ine $L$ and are in contact with the original cylinder on both sides. The upper surface is a part of a right circular cone with apex at $P$, its axis coinciding with the line $L$, and half apex angle, $\pi / 2-\theta_{0}$, such that the conical surface is in contact with the top edge of the cylinder. The bottom surface is on a common plane with the source cylinder. The outer surface is part of a cylinder with its axis on $L$ and radius equal to $a+m_{c} R, m_{c}$ being such that the bottom area of the cylindrical shell fragment is equal to that of the original cylinder."

where

$$
\theta_{0}=\tan ^{-1} \frac{\ell}{a}
$$


$m_{C}=\sqrt{\left(\frac{a}{R}\right)^{2}+\frac{\pi}{\phi_{0}}}-\frac{a}{R}$

and equation (5) is approximated as

$\phi_{p} \simeq \frac{1}{2 \pi \mu_{s}} \int_{0}^{\phi_{0}}\left[G\left(\phi_{0}, \mu \mathrm{t} \sec \phi\right)-G\left(\phi_{0},\left(\mu \mathrm{t} \sec \phi+\mu_{s} R\right)\right)\right] d \phi$

The elevation angle as defined by Equation (8) is measured to the top of the front of the annulus, so that the curved top of the annular sector lies above the top of the cylindrical source which it replaces. Therefore, the source volume is not preserved by this approximation. This helps to make this a conservative approximation.

The volume of the annular sector is then

$v_{a n}=\frac{2}{3} \phi_{0} \frac{l}{a}\left[\left(a+m_{c} R\right)^{3}-a^{3}\right]$

To be useful as a hand calculation tool, equation (9) must be expressed in terms of mathematical functions which may be tabulated in readily usable form.

Then

Define $L_{0}\left(\phi_{0}, \mu t\right)=\frac{\int_{0}^{\phi_{0}} G\left(\phi_{0}, \mu t \sec \phi\right) d \phi}{\phi_{0} G\left(\phi_{0}, \mu t\right)}$

Then eq. (9) becomes the approximation of Ono and Tsuro:

$\phi_{p} \simeq \frac{\phi_{0} S v}{2 \pi \mu_{s}} L_{0}\left(\phi_{0}, \mu t\right) \cdot\left(G\left(\theta_{0}, \mu t\right)-G\left(\theta_{0}, \mu t+\mu_{s} m c_{c} R\right)\right)$ 
1. The Functions of Ono and Tsuro

From Equations (4) and (11) these functions are

(a) $G\left(\theta_{0}, b\right)=\int_{0}^{\theta} \cos \theta e^{-b \cdot \sec \theta} d \theta$

which is usually tabulated in the smoothed (easily interpolated) form

$\bar{G}\left(\theta_{0}, b\right)=\frac{e^{b}}{\theta_{0}} G\left(\theta_{0}, b\right)$

and

(b) $L_{0}\left(\phi_{0}, b\right)=\frac{\int_{0}^{\phi_{0}} \int_{0}^{\frac{\pi}{2}} \cos \theta e^{-b \sec \phi \sec \theta} d \phi d \theta}{\phi_{0} \int_{0}^{\frac{\pi}{2}} \cos \theta e^{-b \sec \theta} d \theta}$

which is inherently in smoothed form. $L_{0}\left(\phi_{0}, b\right)$ gives the ratio of the attenuation of a slab shield of thickness $b$ to the attenuation of an annular shield of the same thickness curved around the detect:or point and concentric with the annular sector source. Figure 4 shows the relation of the slab shield and the annular shield which have just been described.

The definition of $L_{0}\left(\phi_{0}, b\right)$ includes a double integral. Numeric checks have shown that the value of this function is a lmost independent of the upper limit on $\theta$ in Equation (15) and is also largely independent of the source shape behind the shield, provided that the source strength is a constant. The limit $\theta=\frac{\pi}{2}$ is therefore valid, and the use of this function is not 1 imited to the approximation of Ono and Tsuro.

Tabulations of these functions are given in Section VI of this report. 
Any further refinement in source shape or attempt to approximate Equation (1) more closely resuits in mathematical functions which are dependent on at least four parameters. A table of such a function must be voluminous and detailed and is difficult to interpolate. Problems also arise because functions such as $G\left(\theta_{0}, b\right)$ and the Sievert Integral $F\left(\theta_{0}, b\right)$ are extremely weak functions of the upper limit when that limit is large (greater than 60 degrees). The plots in Chapter Nine of Reference (6) illustrate this.

In contrast to this, the $G\left(\theta_{0}, b\right)$ and $L_{0}\left(\phi_{0}, b\right)$ functions defined by Ono and Tsuro involve only two parameters or independent variables each and are easy to use. Anyone using this cylindrical source approximation therefore owes a debt of gratitude to 0 no and Tsuro.

\section{A Sample Problem}

An illustrative sample problem will now be displayed in which the approximate flux formula given in Equation (12) is to be used to find the flux at a detector point $15 \mathrm{~cm}$. from the base of a cylindrical source having a radius of $10 \mathrm{~cm}$. and a height of $40 \mathrm{~cm}$. The source attenuation coefficient is $\mu_{s}=0.1$, the source strength $S_{v}$ is 1.0 , and the intervening slab shield is $1.0 \mathrm{mfp}$ thick. Then

$$
\begin{aligned}
\theta_{0} & =\tan ^{-1} \frac{40}{15}=\tan ^{-1} 2.67=69^{\circ}=1.2 r \\
\phi_{0} & =\sin ^{-1} \frac{10}{25}=\sin ^{-1} .4=23.6^{\circ}=0.41 r \\
m_{C} & =1.65 \text { and } b^{\prime}=b+\mu_{s} m_{c} R=2.6 \\
\Phi & =\frac{1 \times .41}{2 \times 3.14 \times .1} L_{0}(.41,1 .)[G(1.2,1)-G(1.2,2.6)] \\
& =0.656 \times .97[.368 \times .606 \times 1.2-.074 \times .48 \times 1.2] \\
& =0.586[.266-.042]=0.131
\end{aligned}
$$


The flux found by a numeric calculation using the exact flux formula is 0.136 , which is in good agreement with the approximate hand calculation.

\section{Improved Accuracy for the Approximation of Ono and Tsuro}

Table 1 gives the ratios, for a range of problem parameters, of the flux calculated by Equation (12) to the exact flux calculated by Equation (1). The tool for doing these calculations was a special version of the SPAR1 program, which is described in Reference (5). Some of these error ratios are larger than 3.

If the source height $\ell$ is replaced by a smaller number, according to the following algorithm, the two sets of ratios which are the worst in Table 1 are replaced by the two sets of the ratios which are designated as Table 1A. This gives an improved Ono and Tsuro cylindrical source approximation which is conservative by less than a factor of $\mathbf{2 . 1 5}$ for the range of problem parameters covered by Table 1 .

The height of the front of the annular sector which will preserve volume is

$$
\ell^{\prime}=\frac{3}{2} \frac{\pi}{\phi_{0}} \frac{R^{2} \ell a}{\left[\left(a+m_{c} R\right)^{3}-a^{3}\right]} \text {. }
$$

Set $\ell^{\prime \prime}=\operatorname{MIN}\left(2.0 * \ell^{\prime}, \ell\right)$ and

$$
\ell^{\prime \prime \prime}=0.5 *\left(\ell^{\prime \prime}+\ell\right)
$$

Then if $\frac{a}{R}<0.5$ and $\mu_{s} R<5.0$, replace $\ell$ by $\ell^{\prime \prime \prime}$ in Equation (8), and if $\frac{a}{R}<0.5$ and $\mu_{s} R<2.5$ replace $\ell$ by $\ell$ '' in Equation (8). The flux may then be found using Equation (12).

III. Extensions of the Approximation of (Ino and Tsuro - Approximate Formulas for the Flux at Detector Points Outside the Radial and Axial Extensions of a Cylindrical Source with a Slab Shield Parallel to the Source Axis

\section{A. Background}

Exact flux formulas for this situation are given in References (3) and (5). Here, an analogue of the approximate formula of Equation (12) which applies to this situation will be derived and tested. 
The approximation of Ono and Tsuro was derived for lateral detector points and it breaks down badly for detector points located beyond the axial and radial extensions of the source, as shown in Figures (5) and (6).

The reason for this is readily apparent. Equation (12) does not model either the top or bottom of the source properly when the geometry is that of Figure (5). However, by properly altering the heights $h$ and $T=h+l$ in Figure (5), the approximation of Ono and Tsuro may be made the basis for a simple and usable approximation using the superposition method. The flux from a source of height $\ell$ which is a distance $h$ above a lateral detector point is assumed to be equal to the flux from a source of height $T=h+\ell$ minus the flux from a source of height $h$. There are two cases to be considered: The case with an intervening slab shield parallel to the axis of the cylindrical source (Figure 5) and the case with a slab shield perpendicular to the axis (Figure 8 ).

These cases are treated in the next two sections of this report.

B. Case 1 - Slab Shield Parallel to the Source Axis

The approximate flux formula for this case is given by the following algorithms:

Step 1. See Figure 5.

The height of the superimposed cylinder, that is, the height of the cylinder of height $\ell$ combined with the cylinder of height $h$, is

$T=h+\ell$

Find the height $T$ ' which will preserve the volume of this large cylinder when it is replaced by a section of an annulus defined to preserve the basal area. This height is given by

$T^{\prime}=\frac{3}{2} \frac{\pi}{\phi_{0}} \frac{R^{2} T a}{\left[\left(a+m_{c} R\right)^{3}-a^{3}\right]}$

where $\phi_{0}$ and $m_{c}$ are defined by Equations (9) and (10), respectively.

Step 2. Let $T^{\prime \prime}=\operatorname{MIN}\left(2.0 * T^{\prime}, T\right)$ and 


$$
T^{\prime \prime \prime}=0.5 *\left(T^{\prime \prime}+T\right)
$$

Step 3. Let $T_{\text {use }}=T$.

$$
\begin{aligned}
& \text { If } \frac{a}{R}<0.5 \text { and } \mu_{s} R<5.0 \text { and } \frac{l}{a}<0.5 \text {, } \\
& \text { set } T_{\text {use }}=T^{\prime \prime \prime} \text {. } \\
& \text { If } \frac{a}{R}<0.5 \text { and }{ }_{1}{ }_{s} R<2.0 \text { and } \frac{\ell}{a}<0.75 \text {, } \\
& \text { set } T_{\text {use }}=T^{\prime \prime} \text {. }
\end{aligned}
$$

Step 4. The height of the lower cylinder in Figure 4 is $h$. Find the height to preserve this volume, which is

$$
h^{\prime}=h \frac{T^{\prime}}{T} .
$$

Step 5. Let $h^{\prime \prime}=\operatorname{MIN}\left(2.0 * h^{\prime}, h\right)$ and

$$
h^{\prime \prime}=0.5 *\left(h^{\prime \prime}+h\right)
$$

Step 6. Let $h_{\text {use }}=h$

$$
\begin{aligned}
& \text { If } \frac{a}{R}<0.5 \text { and } \mu_{s} R<5.0 \text {, } \\
& \text { set } h_{\text {use }}=h^{\prime \prime \prime} \text {. } \\
& \text { If } \frac{a}{R}<0.5 \text { and } \mu_{s} R<2.5 \text {, } \\
& \text { set } h_{\text {use }}=h^{\prime \prime} \text {. }
\end{aligned}
$$


Step 7. Find

$$
\begin{aligned}
& \theta_{2}=\tan ^{-1} \frac{h_{\text {use }}}{a} \text { and } \\
& \theta_{3}=\tan ^{-1} \frac{T_{\text {use }}}{a}
\end{aligned}
$$

Step 8. The flux from the cylinder of height $\ell$ in Figures 5 and 6 is then given by

$$
\begin{aligned}
{ }^{\Phi} P_{1}=\frac{S_{V} \phi_{0}}{2 \pi \mu_{s}} L_{0}\left(\phi_{0}, b\right)\left\{G\left(\theta_{3}, b\right)\right. & -G\left(\theta_{3}, b+\mu_{s} m_{c} R\right) \\
& -G\left(\theta_{2}, b\right)+G\left(\theta_{2}, b+\mu_{s} m_{c} R\right\} .
\end{aligned}
$$

Step 9. Evaluate the accuracy of the result.

The use of this approximate formula is evaluated in Tables 2 and 3. In Table 2, $H^{\prime}=(h+l) / l$, and Mus is the macroscopic attenuation coefficient of the source, and is the same as $\mu_{S}$ in Equation (24).

Table 2 gives ratios of results calculated by Equation (24) to results calculated by the exact cylindrical source option of the SPAR1 program [Reference (5)] for a wide range of problem geometries and parameters. Table 3 gives the results for the sample problem of Figure 7 calculated by the same two methods.

The accuracy is good for many values of the parameters in Table 2 but this approximation gives low answers for certain combinations of the parametric values as $\mathrm{H}^{\prime} / \ell, \ell / a$ and $b$ increase. The maximum ratios given in this table are less than 3. The low or non-conservative ratios are marked off from the ratios in the range of 0.88 to 3.0 .

These ratios may be used as approximate correction factors for calculations where the problem parameters lie in the range of the parameters used in generating this table. The information in this table is therefore very useful. 
The behavior in which non-conservative or low answers are generated is also seen in Table 3.

The reason for this is ealsy to find: Equation (24) involves only $L_{o}$ and $G$ functions, and as may be seen from Table $1, G\left(\theta_{0}, b\right)$ becomes: a very weak function of $\theta_{0}$ as $\theta_{0}$ and $b$ increase. If both $\theta_{2}$ and $\theta_{3}$ [Equations (22) and (23] are greater than $45^{\circ}$ and $b$ is not small, the parameter values and error ratios of Table 2 must be used as guidelines as to the geometric: arrangements for which Equation (24) is trustworthy.

\section{Case II - Slab Shield Perpendicular to the Source Axis}

The geometry of this source and shield configuration is shown in Figure 8. The applicability of the approximation of Ono and Tsuro to this case is not obvious.

However, if an annular sector can be suitably defined which replaces the cylindrical source, the case of the shield perpendicular to the source axis can also be handled by a superposition method based on the approximation of Ono and Tsuro. This configuration is shown in Figure 9. A simple algorithm for defining and using such a sector is as follows:

Step 1. See Figures 8 and 9.

A usable annular sector for representing this source is defined to have height $=2 R$ and thickness $\ell$ and the distance out to a detector point in the base plane would be $h$, while the distance the detector point lies below the source base is a.

Step 2. Find the volume of the alnnular sector, which is given by

$V_{\text {an }}=\pi(2 R)\left[(H+l)^{2}-h^{2}\right] \cdot \phi_{0} / \pi=2 R \phi_{0}\left[(h+l)^{2}-h^{2}\right]$

and the volume of the cylinder, which is $V_{c y l}=\pi R^{2} l$; and equate these volumes to determine the angle $\phi_{0}$ :

$\phi_{0}=\frac{\pi}{2} \frac{R}{2 h+\ell}$. 
Step 3. If $R \leq \frac{2}{3}(2 h+\ell)$,

proceed to Step 4. Otherwise, this approximation does not apply to the problem. See Step 7 below for an explanation of this criterion.

Step 4. Define $\theta_{2}=\tan ^{-1} \frac{a}{h+l}$ and

$$
\theta_{3}=\tan ^{-1} \frac{a+2 R}{h} \text {. }
$$

Step 5. The flux from the cylinder of height $\ell$ at the point $P_{1}$ is then given by

$$
\begin{aligned}
\Phi_{P_{1}}=\frac{S_{v} \phi_{0}}{2 \pi \mu_{s}} L_{0}\left(\phi_{0}, b\right)\left\{G\left(\theta_{3}, b\right)\right. & -G\left(\theta_{3}, b+\mu_{s} l\right) \\
& \left.-G\left(\theta_{2}, b\right)+G\left(\theta_{2}, b+\mu_{s} l\right)\right\}
\end{aligned}
$$

Step 6. Here again, cases in which $\theta_{2}$ and $\theta_{3}$ are both above $45^{\circ}$ are likely to give low answers and the cautions given at the end of Section III.B for Equation (24) also apply to this approximation.

Step 7. Evaluate the accuracy of the result (see Table 4). This approximation is not applicable to any and all possible variations in the geometry of the source and detector points as noted in Step 3 above.

For practical problems, the angle $\phi_{0}$ (see Figure 9 ) should not be greater than $60^{\circ}$ or $\pi / 3$ radians. The inequality (27) follows from Equation (26). With this added requirement, Equation (27) becomes

$$
\frac{\pi}{3}=\frac{\pi}{2} \frac{R}{2 h+\ell}
$$

so that the maximum value of the ratio $R /(2 h+\ell)$ allowed in the problem using this approximation is 
$\frac{R}{2 h+\ell}=\frac{2}{3}$

or $R \leq \frac{2}{3}(2 h+\ell)$.

The ratios of Table 4 were calculated for problem parameters which observe this restriction. In order to allow the use of all but one of the problem parameters of Table 2 in the generation of the ratios for Table 4 , while observing this restriction, first rewrite Inequality (27) as an equation:

$\frac{R}{\ell}=\frac{2}{3} \frac{(2 h+\ell)}{\ell} ;$ then observe that

$\frac{R}{\ell}=\frac{1}{\left(\frac{\ell}{a}\right)\left(\frac{a}{R}\right)}$, and $\frac{2 h+\ell}{\ell}=2\left(\frac{h+\ell}{\ell}\right)-1$

Substitution in Equation (31) then gives

$\left(\frac{l}{a}\right)=\frac{3}{2} \frac{1}{\left(\frac{a}{R}\right)\left(2 H^{\prime}-1\right)}$

where $H^{\prime}=\frac{h+\ell}{\ell}$.

The minimum values of $H^{\prime}$ and $a / R$ used in compiling Table 2 are 1.1 and 0.2 respectively. These values, when substituted in Equation (32), will give the corresponding minimum value of $\ell / a$ which satisfies the constraint of Inequality (27):

$\left(\frac{l}{a}\right)_{\min }=\frac{3}{2} \frac{1}{0.2[2.2-1]}=6.25$

The other three values of $\left(\frac{a}{R}\right)$ used in compiling Table 2 are $0.5,1.0$, and 2.5 ; these: values, along with $H^{\prime}=1.1$, give the $(\ell / a)$ values $2.5,1.25$, and 0.5 respectively [from Equation (32)]. 
These values of $(\ell / a)$ are the basis for the parameter values used to generate the error ratios of Table 4, which clearly shows the geometrical arrangements for which this approximation is applicable. The restrictions on the problem geometry which apply here clearly affect these ratios.

Note that the maximum ratios are less than 3.0. The low or non-conservative ratios are marked off from the ratios in the range 0.88 to 3.0 .

These ratios may be used as approximate correction factors for flux calculations using this approximate formula as long as the problem parameters lie within the range of the parameters used to generate this table. The information given in this table is therefore very useful.

Table 5 gives a comparison of exact and approximate results for the problem shown in Figure 10. The accuracy here is reasonably good. All the ratios except two are greater than 0.6 .

\section{Approximations of Rectangular Sources}

A. Approximation of a Rectangular Volume Source by a Section of an Annulus

Provided that a rectangular source is long with respect to it base dimensions and has a lateral detector point, either the approximation of Ono and Tsuro or the new approximations discussed in Section III of this report may be adapted to allow calculation of flux or dose rate from such a source.

The geometry of this situation is shown in Figures 11 and 12 . The volume of the rectangular source is

$$
V_{r}=\ell_{1} \ell_{2} l_{3}
$$

and $M_{R}$, the factor which preserves area of the the base is

$$
M_{R}=\sqrt{\left(\frac{a}{l_{3}}\right)^{2}+\frac{l_{1}}{\phi_{0} l_{3}}}-\frac{a}{l_{3}}
$$

where

$$
\phi_{0}=\tan ^{-1} \frac{\ell_{1}}{2 a}
$$


For this approximation to work we11, the rectangular source dimensions should be such that

$$
\ell_{2}>\ell_{1} \text { and } \ell_{2}>\ell_{3} \text {. }
$$

Then Equations (12),(24), and (30) may be used directly, with $M_{R} l_{3}$ substituted for $m_{c} R$ and $\phi_{0}$ defined by Equation (35). The $\theta-1$ imits are defined in the same way as in the discussions of Equations (24) and $(30)$, again with $m_{c} R$ replaced by $M_{R} \ell_{3}$.

The accuracy of this approximation will be similar to that of the approximation of the corresponding cylindrical source case. The closer the shape of the annular siector approaches the shape of the true source, the better the resulis calculated by the approximate formulas.

Approximations of rectangular sources for which the above inequalities are not true are discussed below.

The height $\ell^{\prime}$ which will preserve volume in the situation of Figure 12 is

$$
\ell^{\prime}=\frac{3 a \ell_{1} l_{2} l_{3}}{2 \phi_{0}\left[\left(a+M_{R} l_{3}\right)^{3}-a^{3}\right]}
$$

B. Approximation of Rectangular Source with Square Cross-Section by a Truncated Cone

As shown in Figure 13, the flux at: a point located opposite the center of the square end of a square rectangular source may be found approximately by use of a truncated cone source.

To preserve the area of the square end of the source, the radius $R$ of the near end of the truncated cone should be

$$
R=\frac{\ell_{1}}{\sqrt{\pi}}
$$

and the length of the truncated cone which will preserve source volume is then

$$
l=\left[a^{3}+3 a^{2} l_{3}\right]^{1 / 3}-a
$$


where $V=\ell_{1}^{2} \ell_{3}$ is the rectangular source volume. See Figure 13 for definitions of $a, \ell_{1}$, etc.

The defining angle of the truncated cone is then

$$
\theta_{0}=\tan ^{-1} \frac{\ell_{1}}{\mathrm{a} \sqrt{\pi}} .
$$

If $b=\mu t$ is the optical thickness of the slab shield, $\mu_{s} l$ is the optical thickness of the truncated cone, and

$$
b^{\prime}=b+\mu_{s}^{2} \text {. }
$$

then the flux at the detector point $P_{1}$ is given by

$$
\Phi_{p_{1}}=\frac{S_{V}}{2 \pi \mu_{s}}\left[E_{2}(b)-E_{2}\left(b^{\prime}\right)+\cos \theta_{0} E_{2}\left(b^{\prime} \sec \theta_{0}\right)-\cos \theta_{0} E_{2}\left(b \sec \theta_{0}\right)\right]
$$

\section{Approximation of a Rectangular Surface Source}

A rectangular surface source such as that shown in Figure 14 may be approximated by using a disk source if $\ell_{1} \simeq \ell_{2}$ so that the source is close to being square. For a square surface source represented as a disk, the disk radius which preserves area is

$$
r=\frac{\ell_{1}}{\sqrt{\pi}}
$$
while use of the radius $r=\frac{\ell_{1}}{2} \sqrt{2}=\frac{\ell_{1}}{\sqrt{2}}$ will give a conservative
answer.

In either case,

$$
{ }_{0}=\tan ^{-1} \frac{r}{a}
$$

and given an intervening slab shield of optical thickness $b$, the flux formula is

$$
\Phi_{p}=\frac{S_{a}}{2}\left[E_{1}(b)-E_{1}\left(b \sec \theta_{0}\right)\right]
$$


while if $b=0$, the flux formula is $\phi_{p}=\frac{S_{a}}{2} \log \left(\sec \theta_{0}\right)$.

Now if an intervening slab shield is assumed in Figure 14, the exact equation for the flux at the point $P$ is [from Reference (3)],

$$
\Phi_{p}=\frac{S_{a}}{\pi} \int_{0}^{\theta_{0}} \int_{0}^{\phi_{0}} \sec \phi e^{-b \sec \phi \sec \theta} d \phi d \theta
$$

where

$$
\phi_{0}=\tan ^{-1} \frac{\ell_{1}}{2 a}, \theta_{0}=\tan ^{-1} \frac{\ell_{2}}{2 a \sec \phi}
$$

and the maximum value of $\theta_{0}$ is

$$
\theta_{\mathrm{m}}=\tan ^{-1} \frac{\ell_{2}}{2 \mathrm{a}}
$$

If $\ell_{2}>\ell_{1}$, so that the disk approximation breaks down, a simplified approximation of Equation (45) may be written which uses the $L_{0}\left(\theta_{0}, b\right)$ function of 0 no and Tsuro. This approximation is

$$
\Phi_{p}=\frac{S_{a}}{\pi} \theta_{m} L_{0}\left(\theta_{0}, b\right) \int_{0}^{\phi_{0}} \sec \phi e^{-b \sec \phi_{d \phi}}
$$

and is valid for $\theta_{m}>\phi_{0}$ : Unless $\phi_{0}$ is small with respect to $\theta_{m}$, the actual source shape is unacceptably distorted.

Now the Sievert Integral of the Stcond Kind is defined to be

$$
S_{2}\left(\phi_{0}, b\right)=b \int_{0}^{\phi_{0}} \sec \phi e^{-t \cdot \sec \phi_{d}} d \phi
$$

Therefore, for use in Equation (48), define

$$
F_{2}\left(\phi_{0}, b\right)=\frac{1}{b} S_{2}\left(\phi_{0}, b\right)=\int_{0}^{\phi_{0}} \sec \phi e^{-b \sec \phi_{\phi}} d \phi
$$

Tabulations of this function are given in Section VI of this report. The smoothed form of this function is defined as 


$$
\bar{F}_{2}\left(\phi_{0}, b\right)=\frac{e^{b}}{\phi_{0}} F_{2}\left(\phi_{0}, b\right)
$$

Equation (48) may now be written in the usable form

$$
\Phi_{p}=\frac{S_{a}{ }^{\theta} m}{\pi} L_{0}\left(\theta_{m}, b\right) F_{2}\left(\phi_{0}, b\right)=\frac{S_{a} \theta m}{\pi} L_{0}\left(\theta_{m}, b\right) \phi_{0} e^{-b} \bar{F}_{2}\left(\phi_{0}, b\right)
$$

The function

$$
R\left(\phi_{0}, \ell_{2} / a, b\right)=\int_{0}^{\phi_{0}} \int_{0}^{\theta_{0}} \sec \phi e^{-b \sec \phi \sec \theta} d \phi d \theta
$$

is also tabulated in Section VI. Substitution of this function into the exact rectangular surface source equation [Equation (45)] gives

$$
\Phi_{p}=\frac{S_{a}}{\pi} R\left(\phi_{0}, l / a, b\right)
$$

If $b$ is zero, Equation (48) reduces to

$$
\Phi_{p}=\frac{S_{a}}{\pi} \theta_{m} \log \left(\sec \phi_{0}+\tan \phi_{o}\right)
$$

where $\theta_{\mathrm{m}}$ is the larger angle. Equation (55) is then similar in some ways to Equation (44).

\section{Rectangular Surface Source Sample Problems}

\section{Square Surface Source Problem}

Refer to Figure 14. Assuming an intervening slab shield of optical thickness $b$, the exact equation for the flux at the detector point is

$$
\Phi_{p}=\frac{S_{a}}{\pi} \int_{0}^{\phi_{0}} \int_{0}^{\tan ^{-1} \frac{\ell_{2}}{2 a \sec \phi}} \sec \phi e^{-b \sec \phi \sec \theta} d \phi d \theta
$$


or from Equation (54)

$\Phi_{\mathrm{p}}=\frac{\mathrm{S}_{\mathrm{a}}}{\pi} \phi_{0} e^{-\mathrm{b}} \bar{R}\left(\phi_{0}, \frac{\ell_{2}}{\mathrm{a}}, \mathrm{b}\right)$

since $\bar{R}\left(\phi_{0}, \frac{\ell_{2}}{a}, b\right)=\frac{e^{b}}{\phi_{0}} R\left(\phi_{0}, \ell_{2} / a, b\right)$

where

$\phi_{0}=\tan ^{-1} \frac{\ell_{1}}{2 a}$.

Here, let

$S_{a}=1.0$,

$\mathrm{a}=10.0$,

$\mathrm{b}=2.0 \mathrm{mfp}$, and

$\ell_{1}=\ell_{2}=20$

Then the flux, using the exact rectangular source function $R\left(\phi_{0}, l_{2} / a, b\right)$, is as follows:

$\phi_{0}=\tan ^{-1} \frac{20}{2 \times 10}=\tan ^{-1} 1=45^{\circ}=.7854 r=\frac{\pi}{4}$

$\frac{\ell_{2}}{\mathrm{a}}=\frac{20}{10}=2$. Then $\bar{R}(.7854,2 ., 2)=$..5234 , and

$\Phi_{p}=\frac{\pi / 4}{\pi} \times \frac{1}{\mathrm{e}^{2}} \times 0.5234=0.25 \times .1353 \times .5234=\underline{0.0177}$

If the same problem is solved using the approximation of a disk source with area preserved 


$$
\begin{aligned}
r & =\frac{\ell_{1}}{\sqrt{\pi}}=\frac{20}{1.77}=11.30, \\
\theta_{m} & =\tan ^{-1} \frac{r}{a}=\tan ^{-1} \frac{11.3}{10}=48.50=.846 r
\end{aligned}
$$

$\sec 48.5^{\circ}=1.51$

and

$\Phi_{p}=\frac{S_{a}}{2}\left[E_{1}(b)-E_{1}\left(b \sec \theta_{0}\right)\right]=\frac{1}{2}\left[E_{1}(2)-E_{1}(3.02)\right]$

$\Phi_{p}=\frac{1}{2}\left[\frac{e^{-2}}{2} \bar{E}_{1}(2)-\frac{e^{-3.02}}{3.02} \bar{E}(3.02)\right]=.5\left[.0677 \times .723-\frac{.05}{3.02} \times .786\right]$

$\Phi_{p}=.5[.0359]=.0179$.

If the disk source approximation is changed to be conservative, with $r=\ell_{1} \sqrt{2}$, the calculation of the flux is as follows:

$$
r=\frac{20}{\sqrt{2}}=14.1 \text { and } \theta_{m}=\tan ^{-1} \frac{14.1}{10}=0.955 r=54.6^{\circ}
$$

$\sec \theta_{\mathrm{m}}=\frac{1}{\cos 54.6}=\frac{1}{.579}=1.73$

$$
\begin{aligned}
\Phi_{p} & =\frac{S_{a}}{2}\left[E_{1}(b)-E_{1}\left(b \sec \theta_{m}\right)\right]=\frac{1}{2}\left[\frac{e^{-2}}{2} \bar{E}_{1}(2)-\frac{e^{-3.46}}{3.46} \bar{E}_{1}\right. \\
& =\frac{1}{2}\left[3.0677 \times .723-\frac{0.0314}{3.46} \times 0.8\right]=0.5 \times 0.0417=\underline{0.0208}
\end{aligned}
$$

Finally, if the approximation of Equation (52) is used, even though, for a square surface source,

$\theta_{\mathrm{m}}=\phi_{0}=\tan ^{-1} \ell_{1} / 2 \mathrm{a}$,

the flux is given by 


$$
\begin{aligned}
\Phi_{p} & =\frac{S_{a} \theta_{m}}{\pi} L_{0}\left(\theta_{0}, b\right) \phi_{0} e^{-b} \bar{F}_{2}^{*}\left(\phi_{0}, b\right) \\
& =\frac{1 \times .7854}{\pi} L_{0}(.7854,2) \times .7854 \mathrm{e}^{-2} \bar{F}_{2}(.7854,2) \\
& =.25 \times .7842 \times .7854 \times .1353 \times .8819=\underline{.0185}
\end{aligned}
$$

\section{Rectangular Surface Source Problem}

The convention is that $\ell_{2}$, the vertical dimension, is the longer side. A disk source approximation will not work in this case. The detector point is located as in Figure 14.

Let $\ell_{2}=20 \mathrm{~cm}, \ell_{1}=10 \mathrm{~cm}, b=2 \mathrm{mfp}, a=10 \mathrm{~cm}$, and $S_{a}=1.0$

Then

$$
\begin{aligned}
& \theta_{\mathrm{m}}=.7854 \mathrm{r}=450 \\
& \phi_{0}=\tan ^{-1} \frac{\ell_{1}}{2 \mathrm{a}}=\tan ^{-1} \frac{10}{2 \times 10}=\tan ^{-1} .5=26.50=.463 \mathrm{r} \\
& \ell_{1} / \mathrm{a}=10 / 10=1 .
\end{aligned}
$$

The flux given by the exact formula [see Equations (45) and (54)] is

$$
\begin{aligned}
& \Phi_{p}=\frac{S_{a}}{\pi} \phi_{0} e^{-b} \bar{R}\left(\phi_{0}, a_{2} / a, b\right) \\
& \Phi_{p}=\frac{1}{\pi} .463 \times .1353 \bar{R}(.463,1 ., 2)=0.147 \times .1353 \times .423 \\
& \Phi_{p}=\underline{0.00841}
\end{aligned}
$$

The flux from an approximation founded on integrating a line source over the arc $a \phi_{0}$ is 


$$
\begin{aligned}
\Phi_{p} & =a \phi\left(\frac{4 S_{a}}{4 \pi a}\right) F\left(\theta_{m}, b\right)=\frac{S_{a}}{\pi} \phi_{o} e^{-b} \theta_{m} \bar{F}\left(\theta_{m}, b\right) \\
& =\frac{1 \times .463}{\pi} \times .1353 \times .7854 \bar{F}(.7854,2) \\
& =0.463 \times .1353 \times .25 \times .8038=.01262
\end{aligned}
$$

The flux given by the rectangular surface source approximation of Equation (52) is

$$
\begin{aligned}
\Phi & =S_{a} \frac{\theta_{m}}{\pi} L_{0}\left(\theta_{m}, b\right) \phi_{0} e^{-b} \bar{F}\left(\phi_{0}, b\right) \\
& =1 \times \frac{.7854}{\pi} L_{0}(.7854,2) \times .463 \times .1353 \times \bar{F}_{2}(.463,2) \\
& =.25 \times .7842 \times .463 \times .1353 \times .963=.0119
\end{aligned}
$$




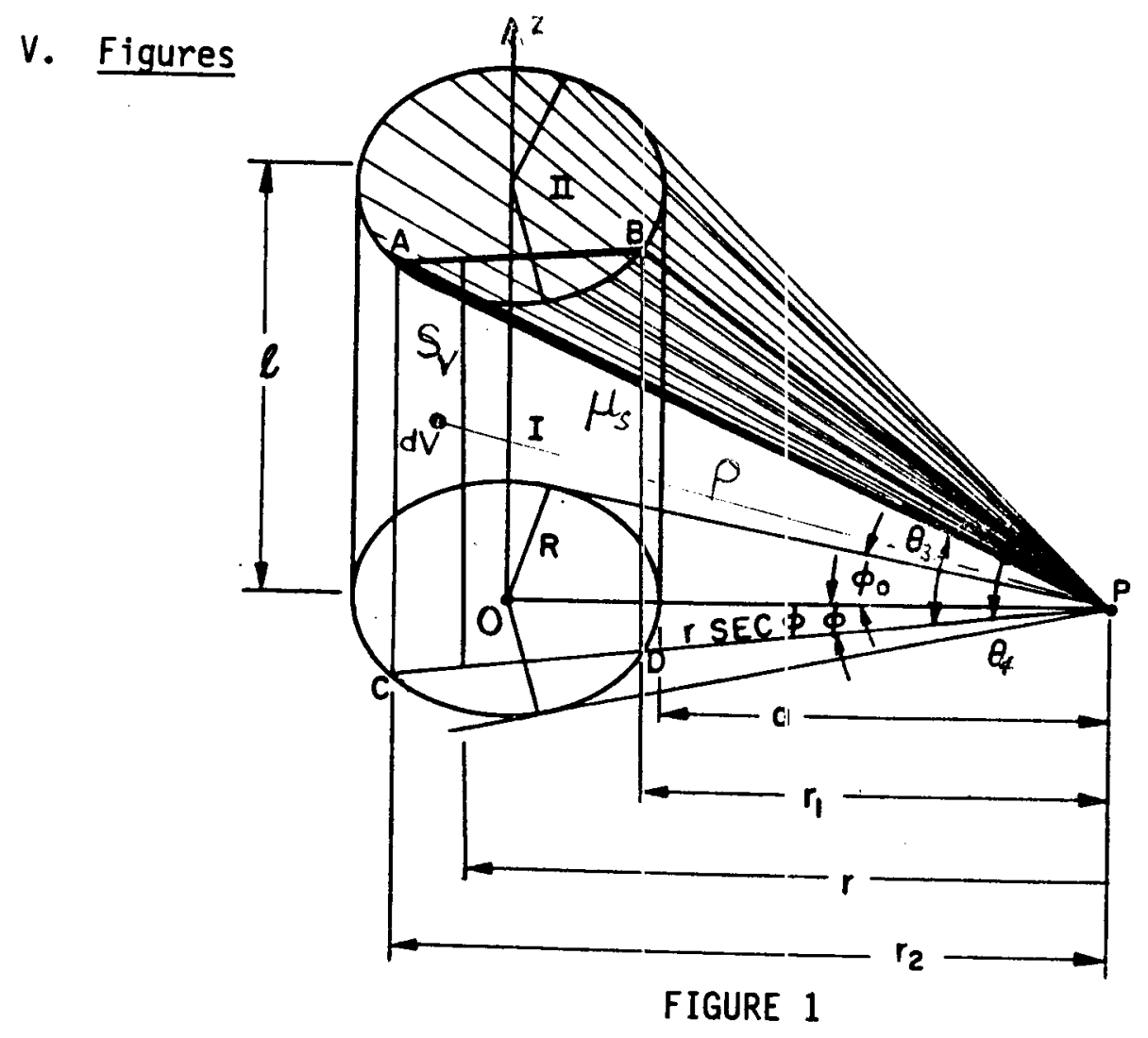

WAPD-TM-1623
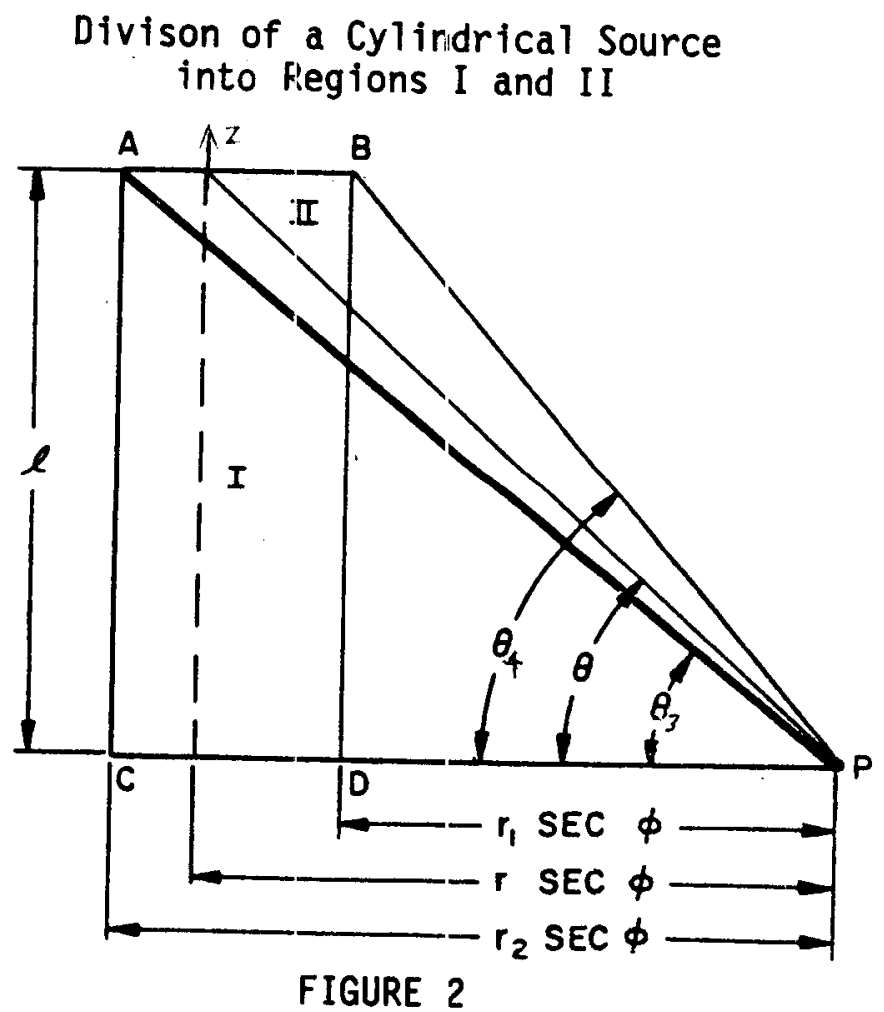

Detail of Division of Cylindrical Source into Regions $I$ and II 
WAPD-TM-1623

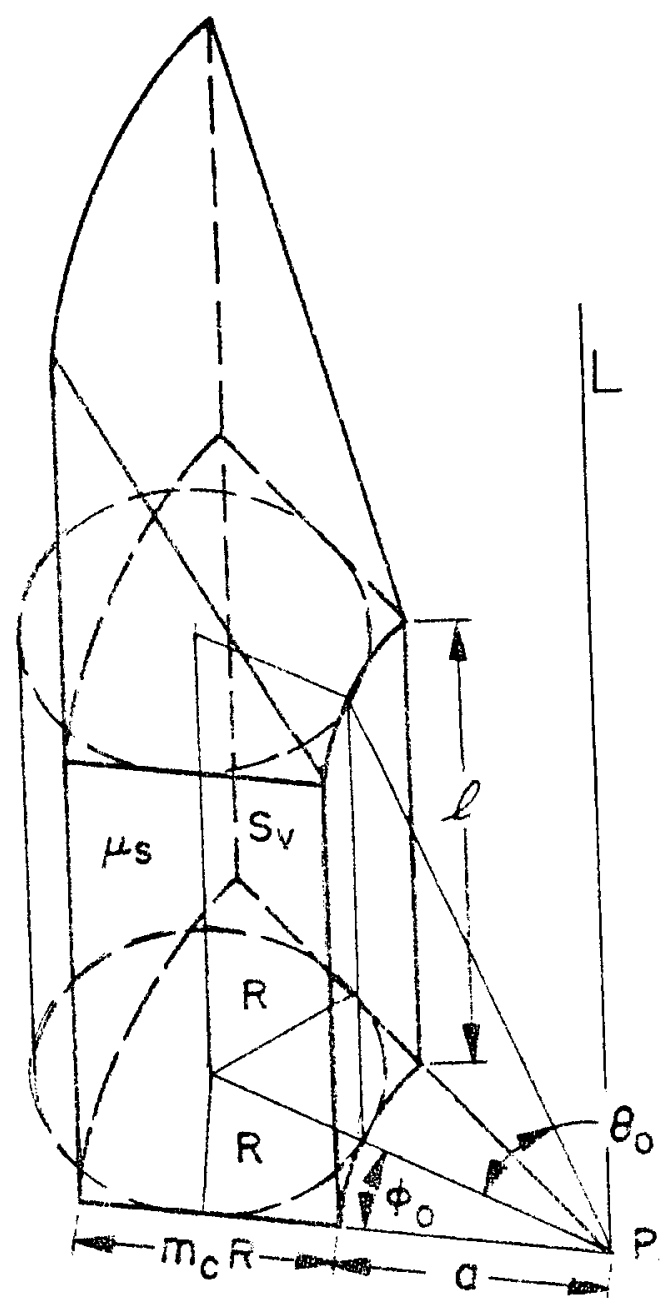

FIGURE 3

Cylindrical Source-Volume Approximation Using a Section of an Annulus with a Curved Top Surface 


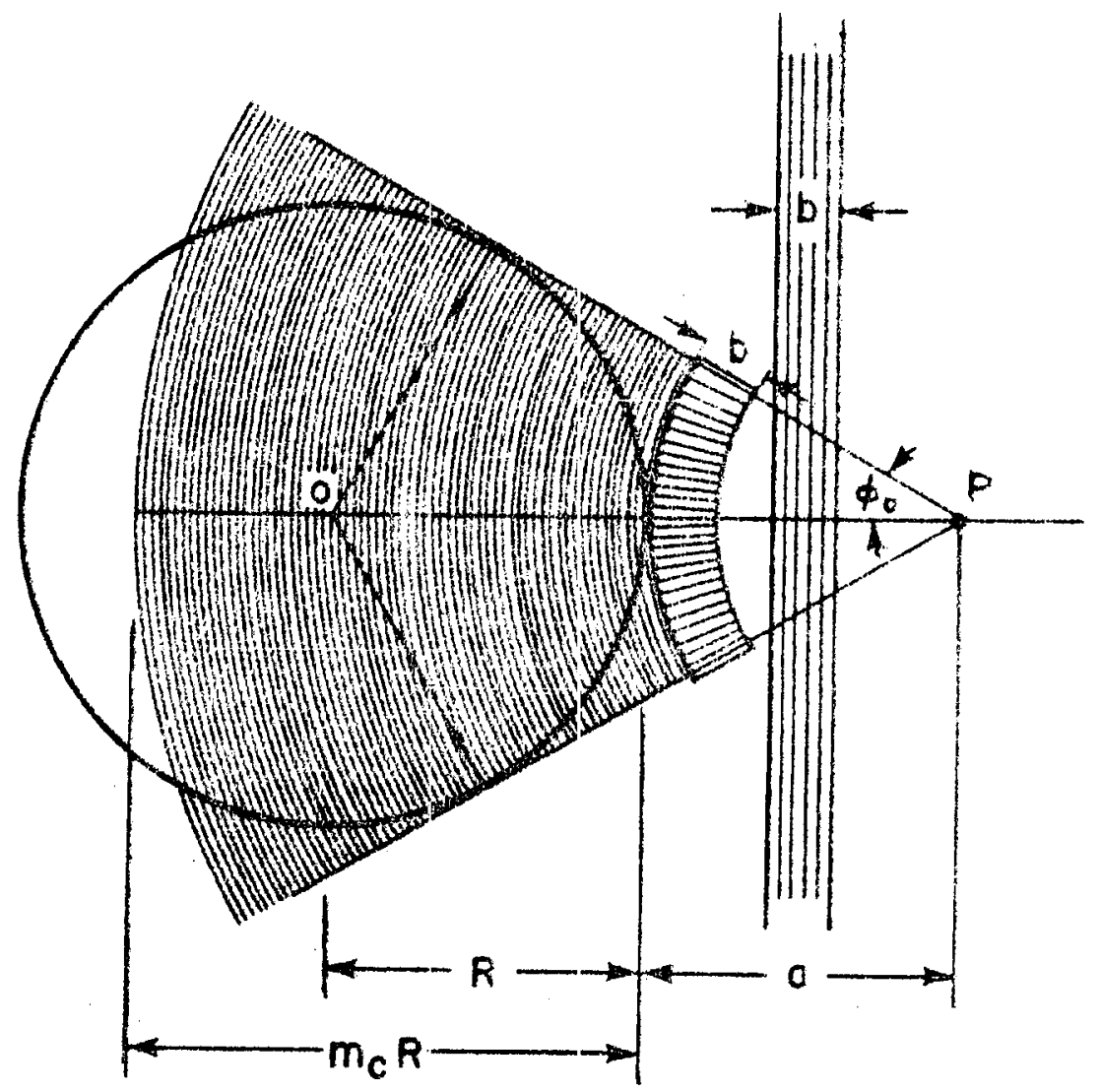

FIGURE 4

Bases of Cylinder and Annular Sector 


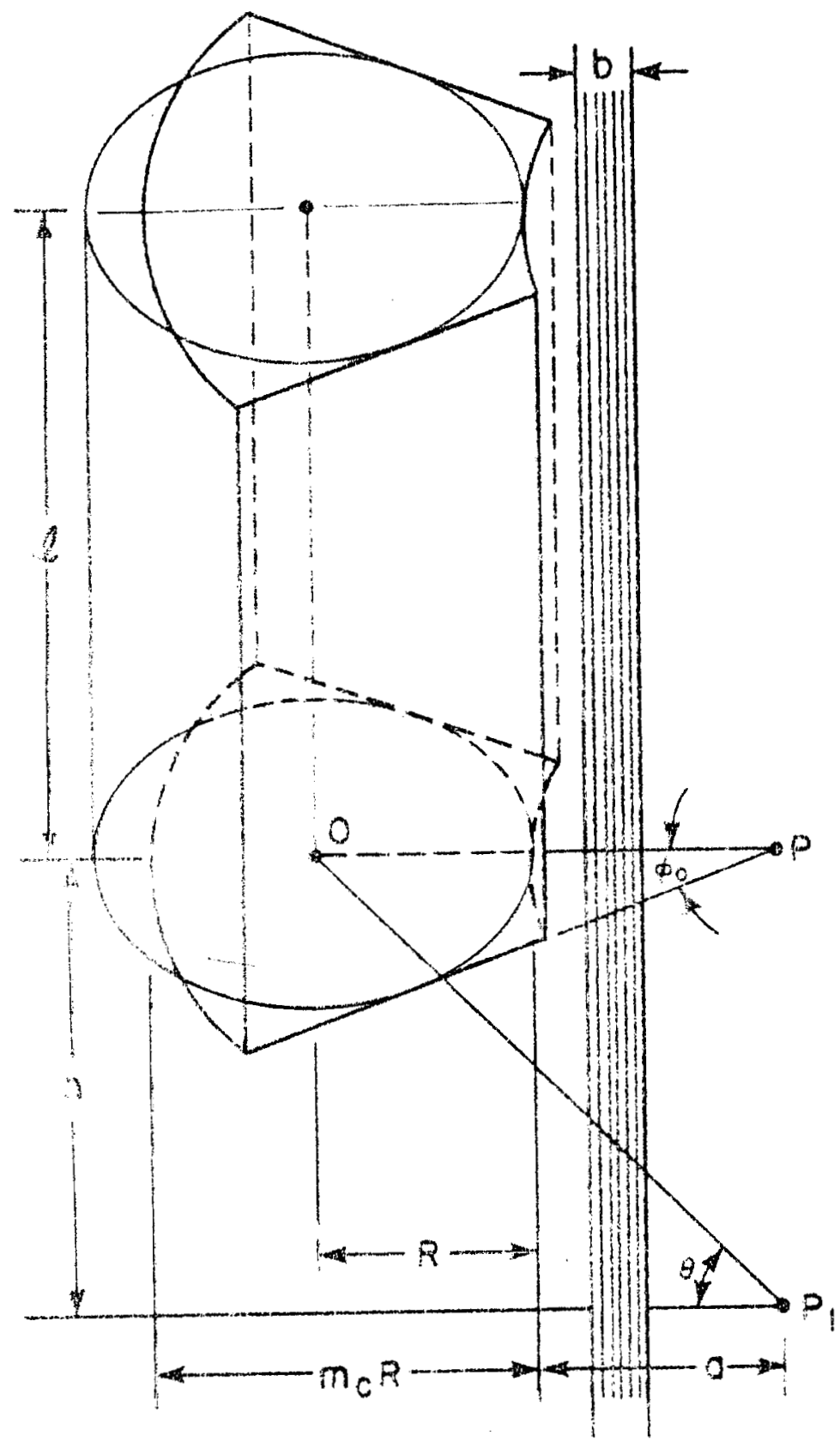

FIGURE 5

Cylindrical Source Approximation with Detector Point Outside the Axial and Radial Extensions of the Source, and with Slab Shield Parallel to the Source Axis

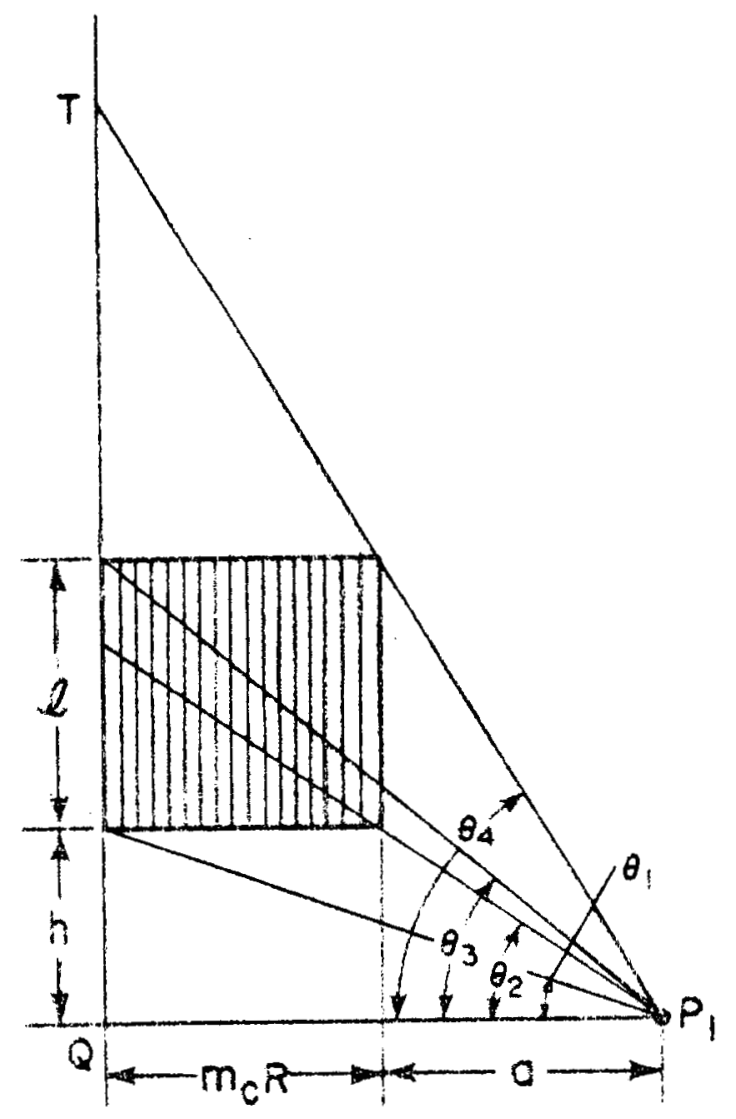

FIGURE 6

Detail of the Midplane of a Cylindrical Source Approximation with a Detector Point Outside the Axial and Radial Extensions of the Source 


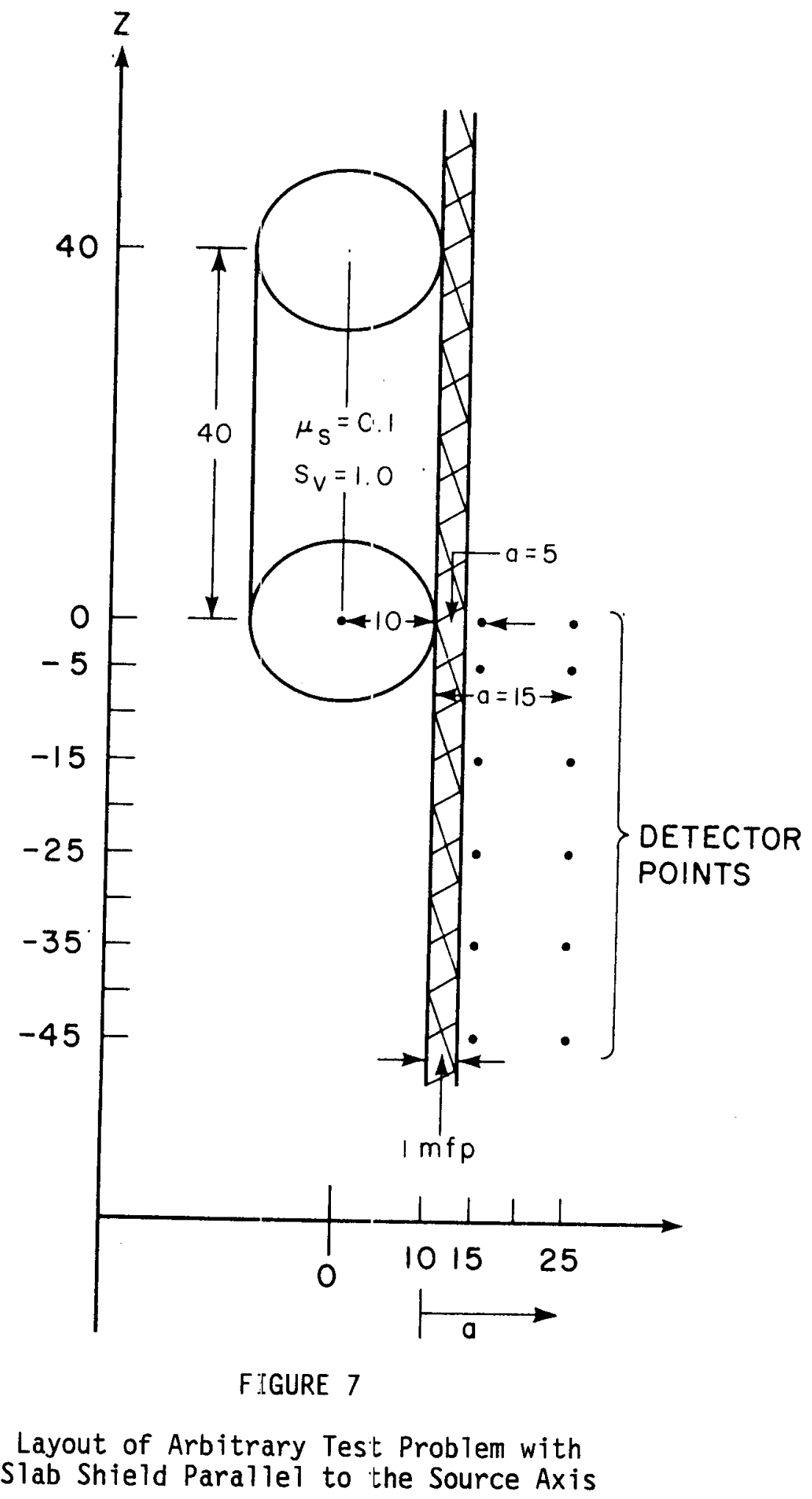




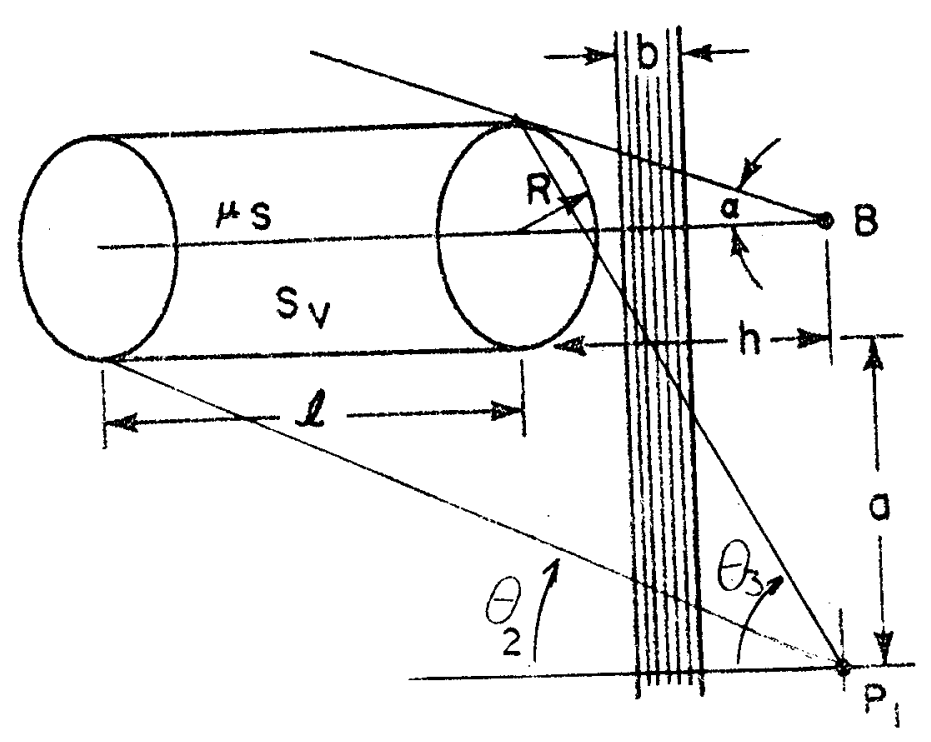

FIGURE 8

Cylindrical Source with a Detector Point Outside the Radial and Axial Extensions of the Source and with a Slab Shield Perpendicular to the Source Axis

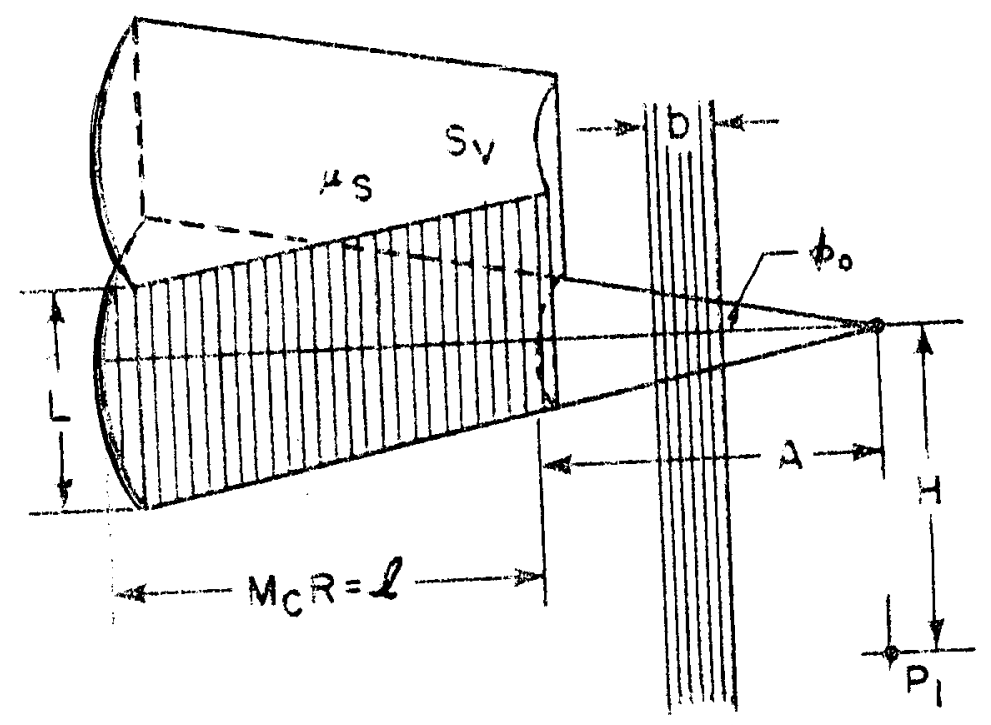

FIGURE 9

For the approximation let

$H=a$,

$A=h$, and

$M_{C}=\frac{\ell}{R}$ so that $M_{C} R=\ell$

Then set $L=2 R$, equate volumes, and soive for $\phi_{0}$ :

$\phi_{0}=\frac{\pi R}{2(2 h+l)}$

Section of an Annulus Used to Approximate a Cylindrical Source with a Detector Point Outside the Radial and Axial Extensions of the Source, and with a Slab Shield Perpendicular to the Source Axis 
WAPD-TM-1623

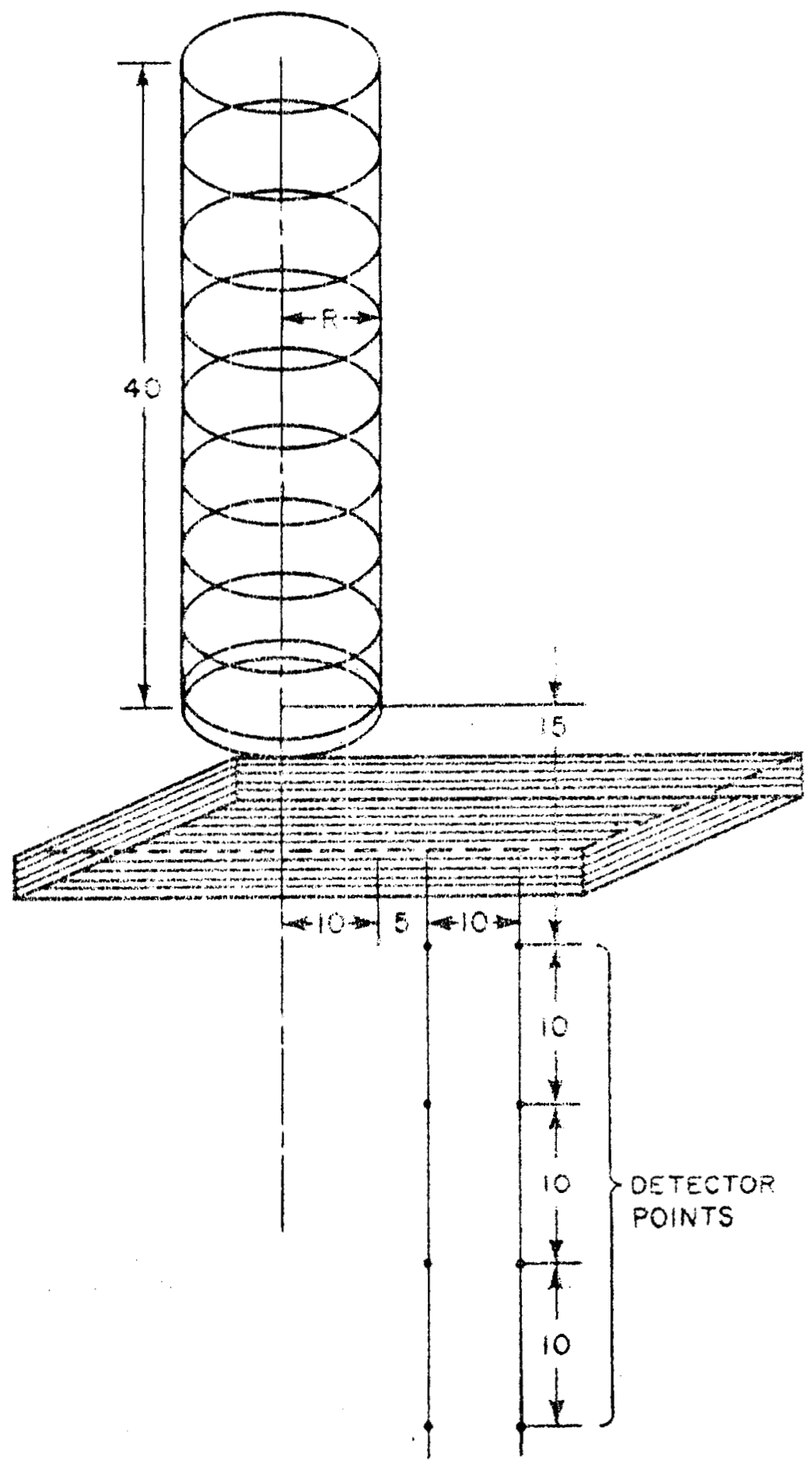

FIGURE 10

Layout of Arbitrary Test Problem with a Slab Shield Perpendicular to the Source Axis 


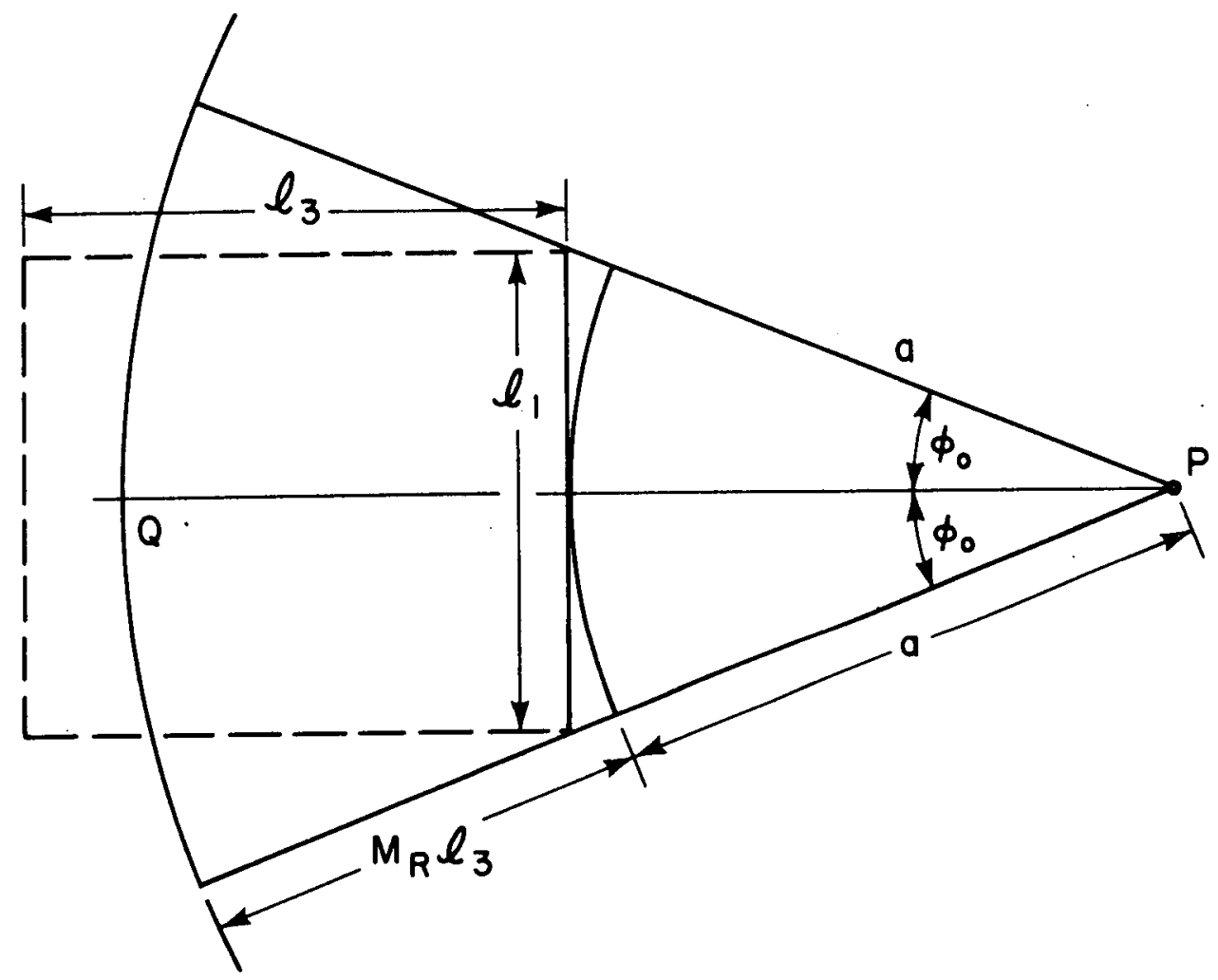

FIGURE 11

Base of an Annular Sector which

Preserves the Area of the Base of a Rectangular Source 


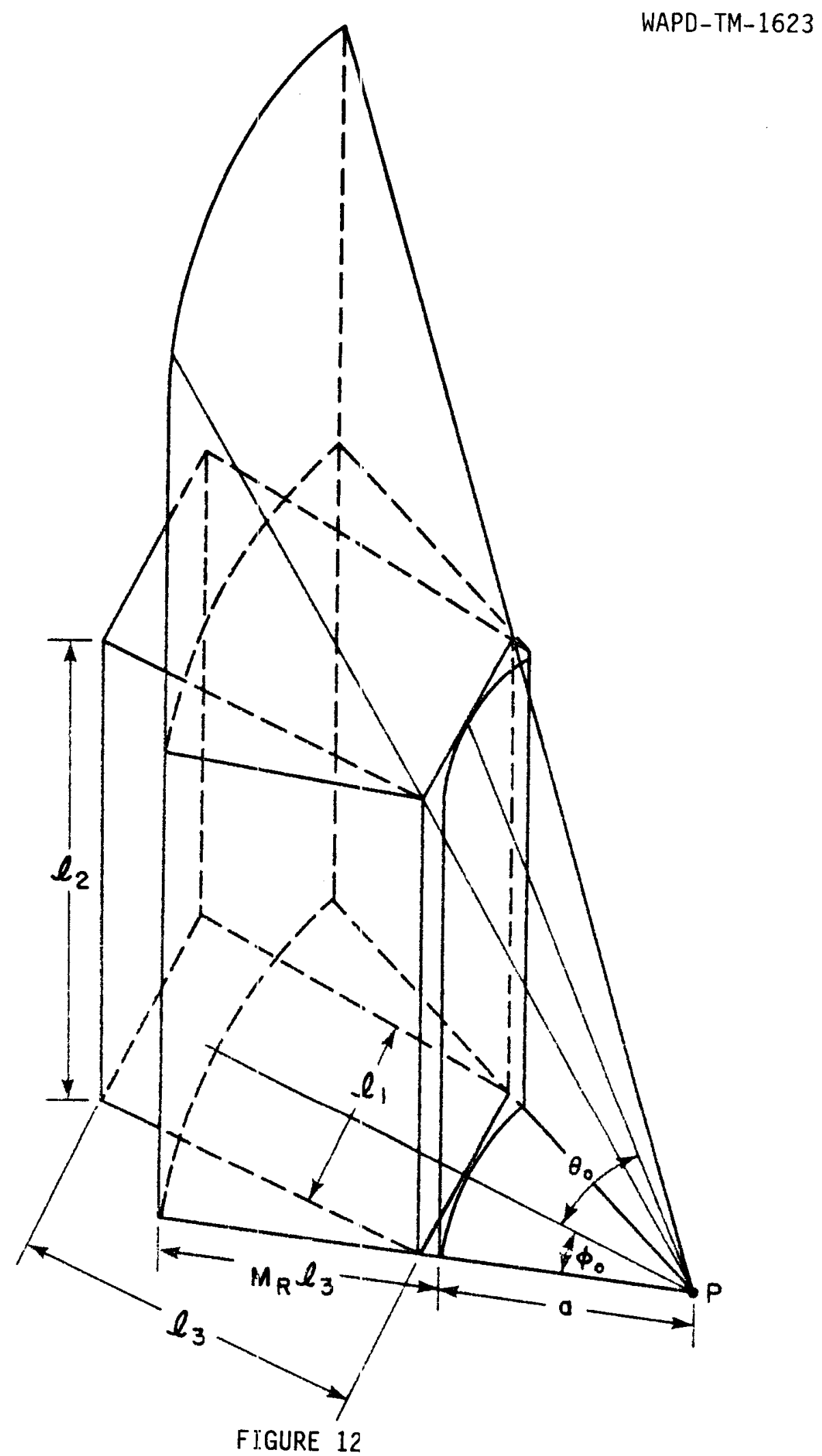

Rectangular Volume Source Approximation Using a Section of an Annulus 


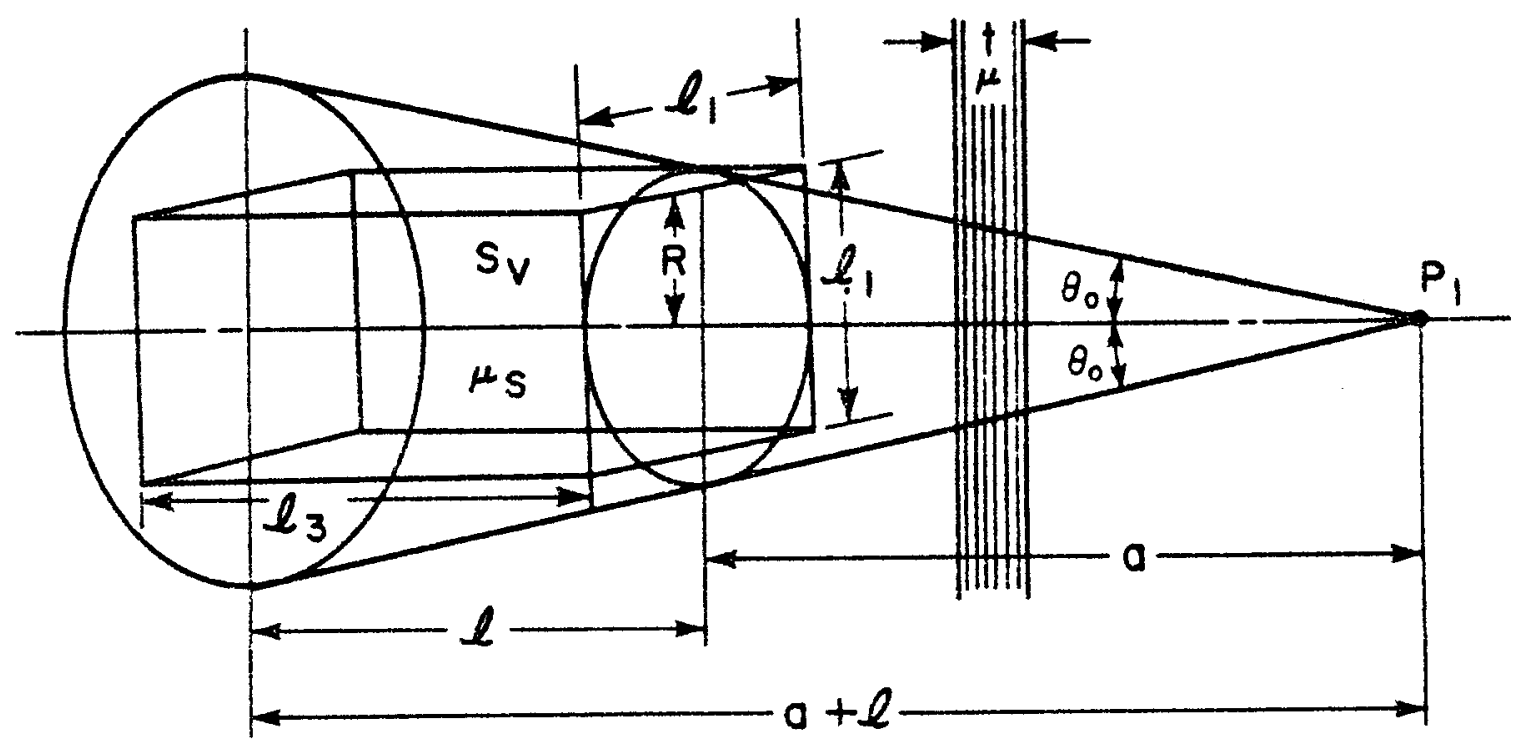

FIGURE 13

Square Rectangular Source Approximation Using a Truncated Cone 


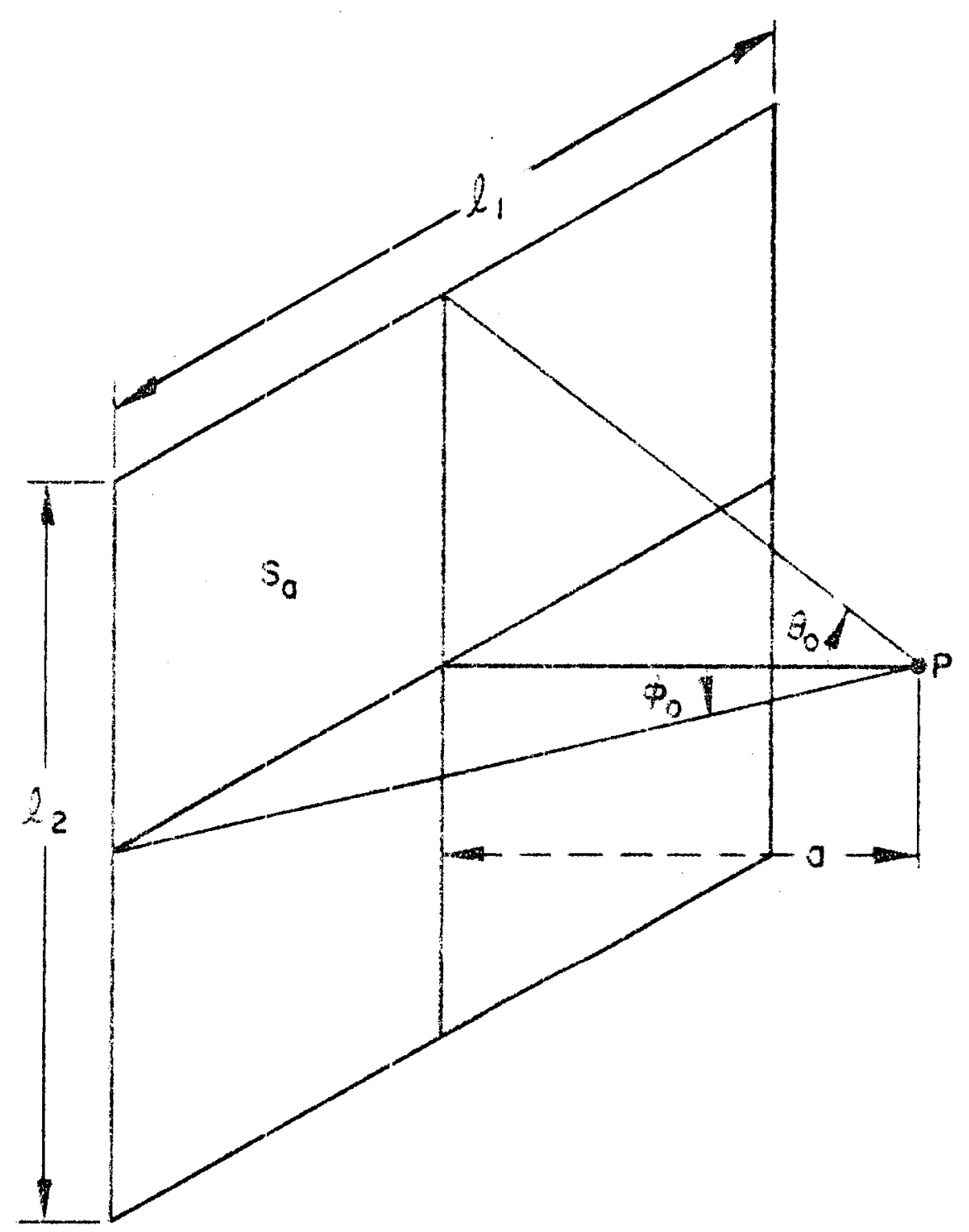

FIGURE 14

Rectangular Surface Source with

Centered Opposite lletector Point 
VI. Tables

TABLE 1

$\frac{\text { Approximate Flux }}{\text { Exact Flux }}$ for a

Cylindrical Source with a

Lateral Detector Point and

a Slab Shield Parallel to the

Source Axis

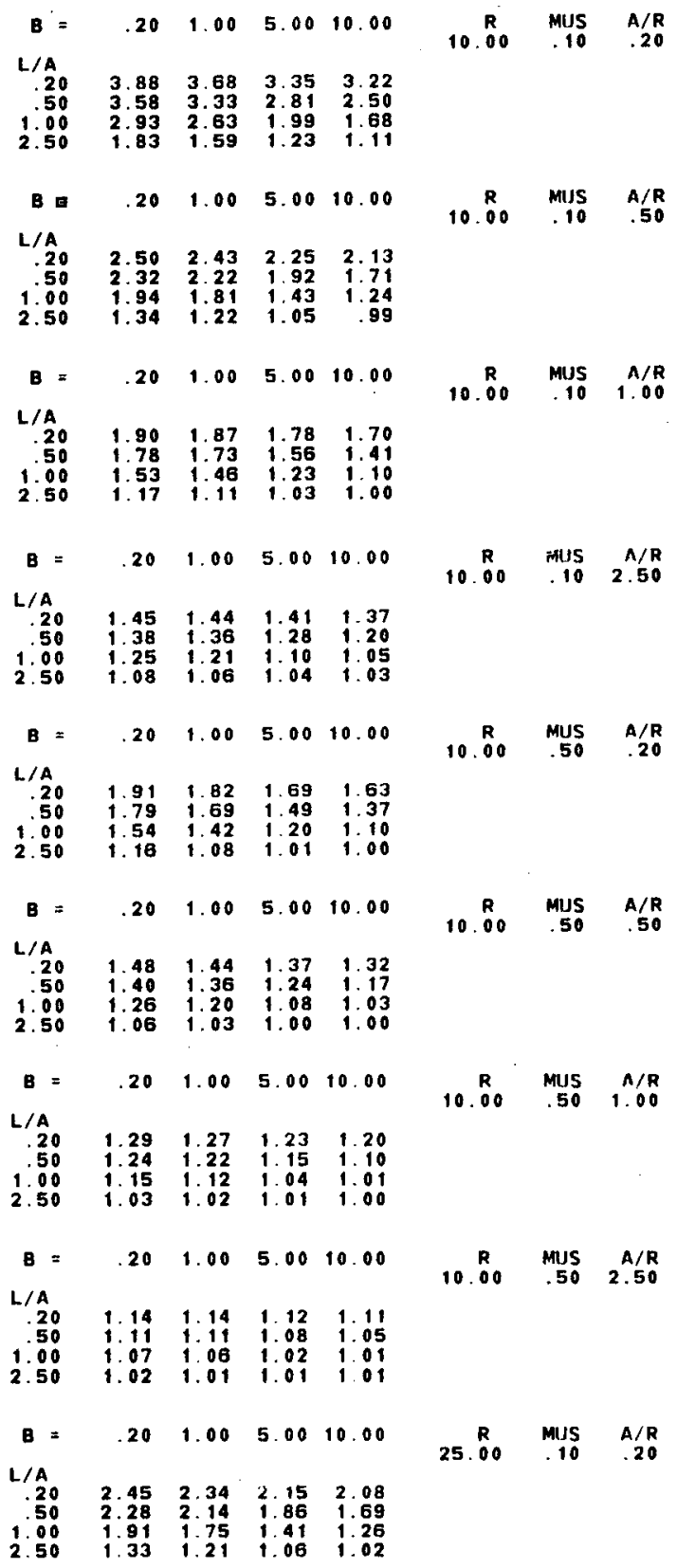

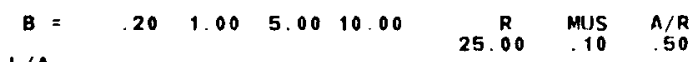
$\begin{array}{rrrrr}\text { L/A } & & & \\ .20 & 1.75 & 1.71 & 1.61 & 1.55 \\ .50 & 1.65 & 1.59 & 1.43 & 1.39\end{array}$
$\begin{array}{lllll}1.50 & 1.65 & 1.59 & 1.43 & 1.31 \\ 2.50 & 1.44 & 1.36 & 1.17 & 1.08 \\ & 1.13 & 1.07 & 1.01 & 1.00\end{array}$
$B=.20 \quad 1.00 \quad 5.00 \quad 10.00$
$\begin{array}{lllll}\text { L/A } & & & & \\ .20 & 1.45 & 1.43 & 1.38 & 1.34 \\ .50 & 1.38 & 1.35 & 1.26 & 1.18 \\ 1.00 & 1.25 & 1.20 & 1.09 & 1.04\end{array}$
$\begin{array}{ccc}R & \text { MUS } & \text { A/R } \\ 25.00 & .10 & 1.00\end{array}$
$\begin{array}{lllll}.50 & 1.38 & 1.35 & 1.26 & 1.18 \\ 1.00 & 1.25 & 1.20 & 1.09 & 1.04 \\ 2.50 & 1.07 & 1.04 & 1.02 & 1.01\end{array}$
$B=\begin{array}{lllllllll}.20 & 1.00 & 5.00 & 10.00 & R & \text { MUS } & \text { A/R } \\ & & & & & 25.00 & .10 & 2.50\end{array}$
$\begin{array}{lllll}\text { L/A } & & & \\ .20 & 1.22 & 1.22 & 1.20 & 1.19 \\ .50 & 1.19 & 1.18 & 1.13 & 1.10\end{array}$
$\begin{array}{lllll}1.00 & 1.12 & 1.10 & 1.05 & 1.03 \\ 2.50 & 1.04 & 1.03 & 1.02 & 1.02\end{array}$

$\begin{array}{ccc}R & \text { MUS } & \text { A/R } \\ 25.00 & .50 & .20\end{array}$

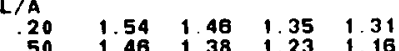

$\begin{array}{lllll}1.50 & 1.46 & 1.38 & 1.23 & 1.16 \\ 1.00 & 1.30 & 1.21 & 1.07 & 1.02 \\ 2.50 & 1.07 & 1.03 & 1.00 & 1.00\end{array}$

$0.201 .00 \quad 5.0010 .00$

$\begin{array}{ccc}R & \text { MUS } & \text { A/R } \\ 25.00 & .50 & .50\end{array}$

$\begin{array}{lllll}\text { L/A } & 1.29 & 1.26 & 1.20 & 1.16\end{array}$

$\begin{array}{rrrrr}.50 & 1.25 & 1.21 & 1.13 & 1.08 \\ 1.00 & 1.15 & 1.1 & 1.03 & 1.01 \\ 2.50 & 1.03 & 1.01 & 1.00 & 1.00\end{array}$

$B=.20 \quad 1.00 \quad 5.001000$

$25.00 \quad M \quad 50 \quad$ A.R

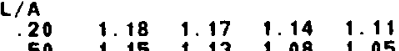

$\begin{array}{rrrrr}.50 & 1.15 & 1.13 & 1.08 & 1.05 \\ 1.00 & 1.08 & 1.07 & 1.02 & 1.00 \\ 2.50 & 1.02 & 1.01 & 1.00 & 1.00\end{array}$

$B=$\begin{tabular}{llllllll}
20 & 1.00 & 5.00 & 10.00 & $R$ & MUS & $A / R$ \\
\hline & & & & & 25.00 & .50 & 2.50
\end{tabular}

$\begin{array}{lllll}\text { L/A } & 1.09 & 1.09 & 1.08 & 1.07\end{array}$

$\begin{array}{rrrrr}.50 & 1.07 & 1.07 & 1.05 & 1.03 \\ 1.00 & 1.04 & 1.03 & 1.01 & 1.00 \\ 2.50 & 1.01 & 1.00 & 1.00 & 1.00\end{array}$ 
WAPD-TM-1623

\section{TABLE 1A}

Improved Ratios of

Approx imate Flux

Exact Flux

Using the Algorithm of Section II.D

$\begin{array}{crrrr}8= & .20 & 1.00 & 5.00 & 10.00 \\ L / A & & & & \\ .20 & 1.07 & 1.02 & .95 & .93 \\ .50 & 1.08 & 1.03 & .98 & .99 \\ 1.00 & 1.10 & 1.06 & 1.05 & 1.07 \\ 2.50 & 1.14 & 1.12 & 1.07 & 1.03\end{array}$

$B=\quad .20 \quad 1.00 \quad 5.00 \quad 10.00$

$\begin{array}{rrrrr}\text { L/A } & & & & \\ .20 & 2.11 & 2.05 & 1.90 & 1.81 \\ .50 & 1.99 & 1.91 & 1.87 & 1.49 \\ 1.00 & 1.73 & 1.61 & 1.30 & 1.14\end{array}$

$\begin{array}{lllll}2.50 & 1.28 & 1.17 & 1.02 & .98\end{array}$

$\begin{array}{ccccc}8 & .20 & 1.00 & 5.00 & 10.00 \\ L / A & & & & \\ .20 & 1.66 & 1.64 & 1.56 & 1.49 \\ .50 & 1.58 & 1.54 & 1.40 & 1.28 \\ 1.00 & 1.42 & 1.35 & 1.16 & 1.06 \\ 2.50 & 1.15 & 1.10 & 1.03 & 1.00\end{array}$

$B=.20 \quad 1.00 \quad 5.00 \quad 10.00$

$\begin{array}{llllll}\text { L/A } & & & & \\ .20 & 1.32 & 1.32 & 1.29 & 1.27 \\ .50 & 1.28 & 1.27 & 1.20 & 1.15 \\ 1.50 & 1.20 & 1.17 & 1.09 & 1.04\end{array}$

$\begin{array}{llllll}1.00 & 1.20 & 1.27 & 1.09 & 1.04 \\ 2.50 & 1.08 & 1.06 & 1.04 & 1.03\end{array}$

$B=.20 \quad 1.00 \quad 5.0010 .00$

$\begin{array}{llllll}\text { L/A } & .20 & 1.91 & 1.82 & 1.69 & 1.63\end{array}$

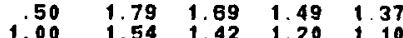

$\begin{array}{lllll}1.00 & 1.54 & 1.42 & 1.20 & 1.10 \\ 2.50 & 1.16 & 1.08 & 1.01 & 1.00\end{array}$

$B=\quad .20 \quad 1.00 \quad 5.00 \quad 10.00$

$\begin{array}{cccccc}\text { L/A } & & & & \\ .20 & 1.48 & 1.44 & 1.37 & 1.32 \\ .50 & 1.40 & 1.36 & 1.24 & 1.17 \\ 1.50 & 1.08 & 1.03 & 1.00 & 1.00\end{array}$

$\begin{array}{rrrrrr}.50 & 1.40 & 1.36 & 1.24 & 1.17 \\ 1.00 & 1.28 & 1.20 & 1.08 & 1.03 \\ 2.50 & 1.08 & 1.03 & 1.00 & 1.00\end{array}$

$\begin{array}{ccccc}B= & .20 & 1.00 & 5.00 & 10.00 \\ L / A & & & & \\ .20 & 1.29 & 1.27 & 1.23 & 1.20 \\ .50 & 1.24 & 1.22 & 1.15 & 1.10 \\ 1.00 & 1.15 & 1.12 & 1.04 & 1.01\end{array}$

$\begin{array}{rrrrr}1.50 & 1.24 & 1.22 & 1.15 & 1.10 \\ 1.00 & 1.15 & 1.12 & 1.04 & 1.01 \\ 2.50 & 1.03 & 1.02 & 1.01 & 1.00\end{array}$

$B=.20 \quad 1.00 \quad 5.00 \quad 10.00$

$\begin{array}{lllll}\text { L/A } & & & & \\ 50 & 1.14 & 1.14 & 1.12 & 1.11\end{array}$

$\begin{array}{rllll}.50 & 1.11 & 1.19 & 1.08 & 1.11 \\ 1.00 & 1.07 & 1.06 & 1.02 & 1.05 \\ 2.50 & 1.02 & 1.01 & 1.01 & 1.01\end{array}$

$B=\quad .20 \quad 1.00 \quad 5.00 \quad 10.00$

$\begin{array}{rrrrrr}\text { L/A } & & & & \\ .20 & 1.62 & 1.55 & 1.45 & 1.43 \\ 50 & 1.59 & 1.52 & 1.41 & 1.37 \\ 1.00 & 1.50 & 1.42 & 1.28 & 1.21 \\ 2.50 & 1.23 & 1.17 & 1.05 & 1.02\end{array}$

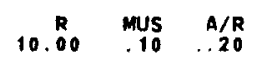

$\begin{array}{ccc}R & \text { MUS } & \text { A/R } \\ 10.00 & .50 & 2.50\end{array}$

$\begin{array}{ccc}R & \text { MUS } & A / R \\ 25.00 & .10 & .20\end{array}$
$\begin{array}{cccccccc}B= & .20 & 1.00 & 5.00 & 10.00 & 25 & \text { MUS } & \text { A/R } \\ L / A & & & & & 25.00 & .10 & \\ .50 & 1.73 & 1.69 & 1.59 & 1.53 & & & \end{array}$

$\begin{array}{rrrrr}.50 & 1.83 & 1.58 & 1.41 & 1.30 \\ 1.00 & 1.43 & 1.35 & 1.18 & 1.07 \\ 2.50 & 1.13 & 1.07 & 1.01 & 1.00\end{array}$

$B=.20 \quad 1.00 \quad 5.00 \quad 10.00$

$\begin{array}{rrrrrr}\text { L/A } & & & & \\ .20 & 1.44 & 1.42 & 1.37 & 1.33 \\ .50 & 1.37 & 1.34 & 1.25 & 1.17\end{array}$

25.00 MUS $\quad .10 \quad \begin{gathered}A / R \\ 1.00\end{gathered}$

$\begin{array}{rrrrrr}1.00 & 1.37 & 1.34 & 1.25 & 1.17 \\ 2.50 & 1.07 & 1.04 & 1.09 & 1.03 \\ & & & & \end{array}$

$B=\begin{array}{llllllll}20 & 1.00 & 5.00 & 10.00 & 25.00 & \text { MUS } \\ \text {.10 } & 2.50 & \text { A/R }\end{array}$

$\begin{array}{llllll}\text { L/A } & & & & & \\ .20 & 1.22 & 1.21 & 1.20 & 1.18 \\ 5 & 1.18 & 1.17 & 1.13 & 1.09\end{array}$

$\begin{array}{lllll}1.00 & 1.18 & 1.17 & 1.13 & 1.09 \\ 2.50 & 1.04 & 1.00 & 1.05 & 1.03\end{array}$

$\begin{array}{lllllllll}B & = & .20 & 1.00 & 5.00 & 10.00 & 25 & \text { MUS } & A / R \\ L / A & & & & & & 25.00 & .50 & \begin{array}{l}.20 \\ .20\end{array}\end{array}$

$\begin{array}{rrrrr}.20 & 1.54 & 1.48 & 1.35 & 1.31 \\ 50 & 1.46 & 1.38 & 1.23 & 1.16 \\ 1.00 & 1.30 & 1.21 & 1.07 & 1.02\end{array}$

$\begin{array}{lllll}1.00 & 1.30 & 1.21 & 1.07 & 1.02 \\ 2.50 & 1.07 & 1.03 & 1.00 & 1.00\end{array}$

$B=\begin{array}{llllllll}B & 20 & 1.00 & 5.00 & 10.00 & 25.00 & \text { MUS } & \text { A/R } \\ L / A & & & & & .50\end{array}$

$\begin{array}{rrrrr}.20 & 1.29 & 1.25 & 1.20 & 1.16 \\ .50 & 1.25 & 1.21 & 1.13 & 1.08 \\ 1.00 & 1.55 & 1.08 & 1.00 & 1.00\end{array}$

$\begin{array}{llllll}1.00 & 1.55 & 1.11 & 1.03 & 1.08 \\ 2.50 & 1.03 & 1.01 & 1.00 & 1.00\end{array}$

$B=\begin{array}{llllllll}20 & 1.00 & 5.00 & 10.00 & 25.00 & \text { MUS } & \text { M. } & \text { M/R } \\ 1.00\end{array}$

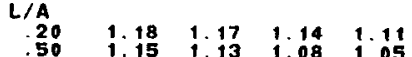

$\begin{array}{lllll}1.00 & 1.55 & 1.13 & 1.08 & 1.05 \\ 2.50 & 1.02 & 1.07 & 1.02 & 1.00 \\ 2.01 & 1.00 & 1.00\end{array}$

$B=\quad .20 \quad 1.00 \quad 5.00 \quad 10.00$

\begin{tabular}{ccc}
$R 5.00$ & MUS & A/R \\
\hline .50 & 2.50
\end{tabular}

$\begin{array}{rrrrrr}1 / A & & & & \\ .20 & 1.09 & 1.09 & 1.08 & 1.07 \\ .50 & 1.07 & 1.07 & 1.05 & 1.03 \\ 1.00 & 1.04 & 1.03 & 1.01 & 1.00 \\ 2.50 & 1.01 & 1.00 & 1.00 & 1.00\end{array}$ 
TABLE 2

$\frac{\text { Approximate Flux }}{\text { Exact Fiux }}$

from A Cylindrical Source at Detector Points Outslde the Radial and Axtal Extenstons of the Source with an Intervening Slab Shield

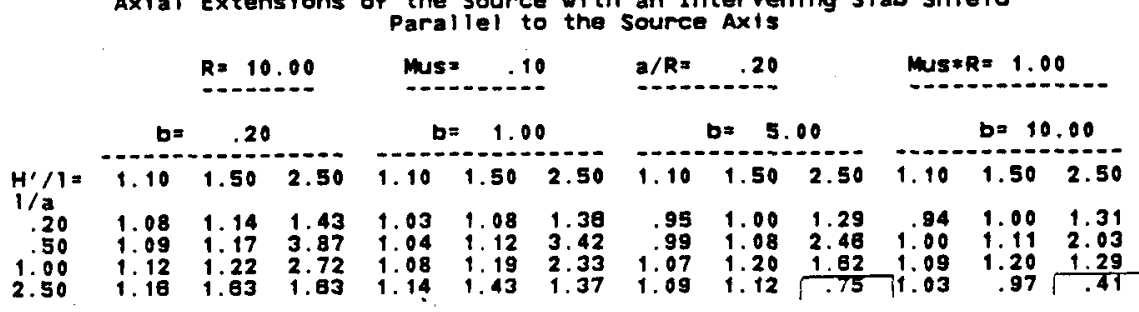

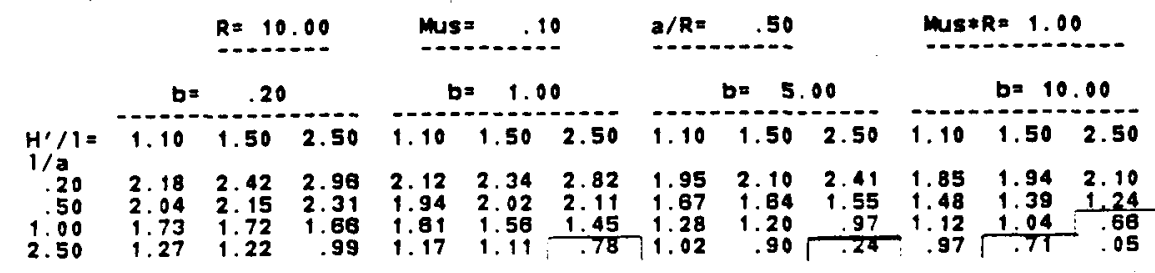

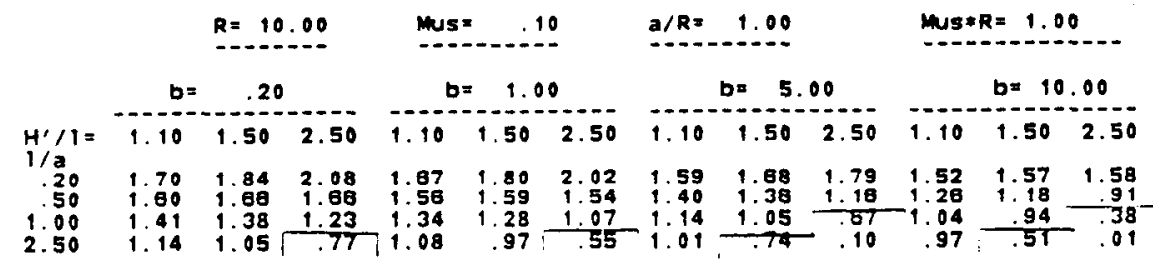

$$
R=10.00 \quad \text { Mus }=.10 \quad a / R=2.50 \quad \text { Mus*R= } 1.00
$$

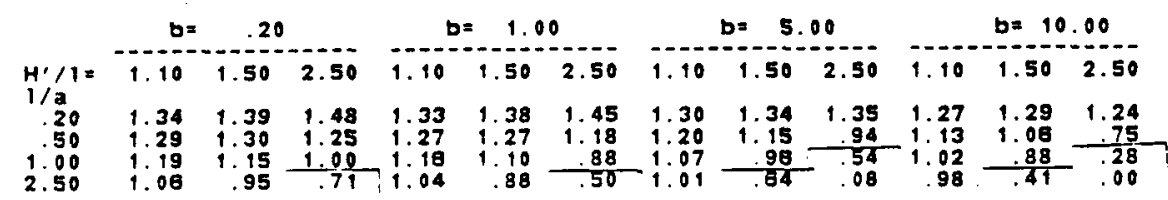
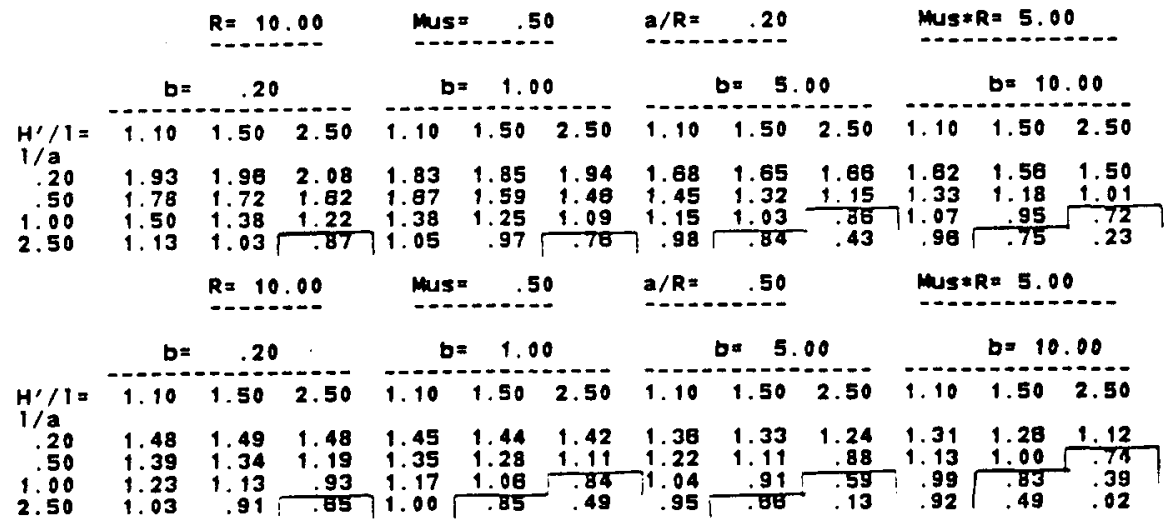

$$
\begin{aligned}
& R=10.00 \quad \text { Mus }=.50 \quad a / R=1.00 \quad \text { Mus*R= } 5.00
\end{aligned}
$$

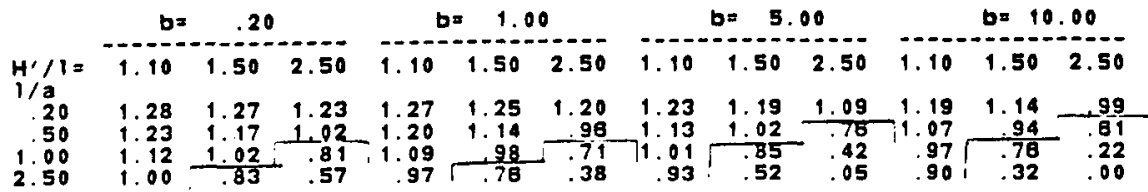

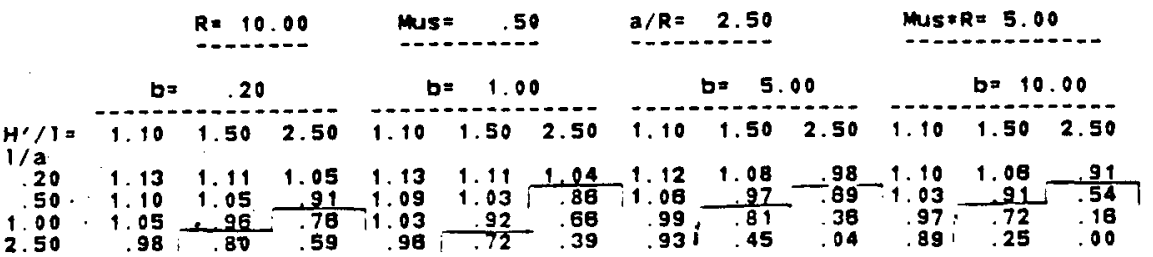




\section{TABLE 2 (Cont'd)}
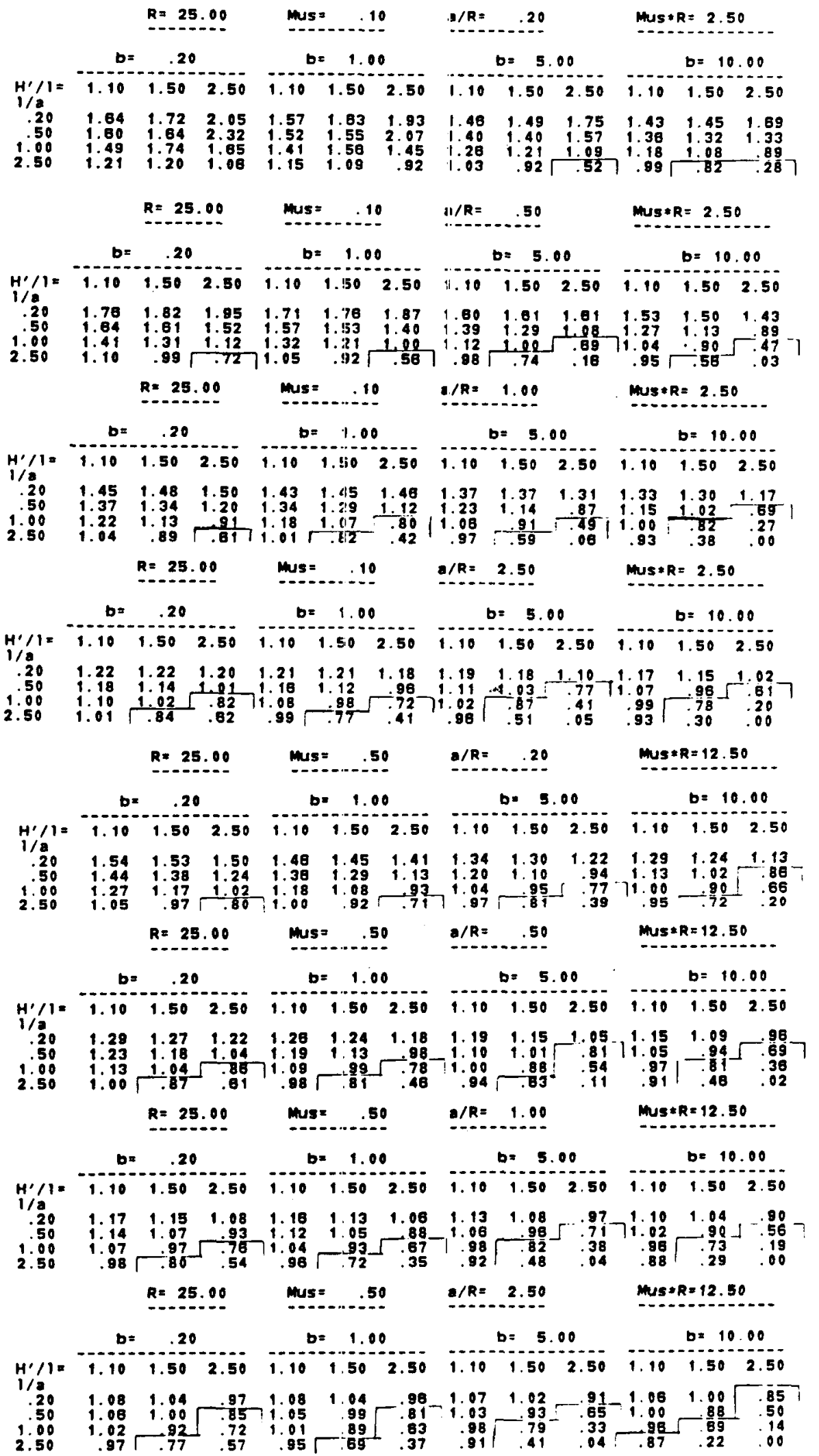


\section{TABLE 3}

Results From the Exact Flux Formula and From Equation (24) for the Problem of Figure 7

$$
u_{s}=0.1 \quad S_{V}=1.0 \quad R=10 .
$$

\begin{tabular}{|c|c|c|c|c|c|c|c|}
\hline \multirow{3}{*}{$\begin{array}{l}\text { Distance of } \\
\text { Detector } \\
\text { Point From } \\
\text { Source Edge } \\
\mathrm{a} \\
\end{array}$} & \multirow{3}{*}{ 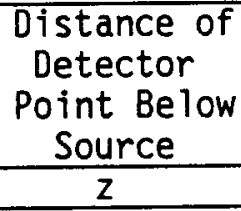 } & \multicolumn{6}{|c|}{ Slab Shield Thickness } \\
\hline & & \multicolumn{2}{|c|}{$\frac{b=0.0}{\text { Flux }}$} & \multicolumn{2}{|c|}{$\begin{array}{c}\mathrm{b}=1.0 \\
\text { Flux }\end{array}$} & \multicolumn{2}{|c|}{$\begin{array}{c}b=5.0 \\
\text { Flux }\end{array}$} \\
\hline & & Exact & Approx & Exact & Approx & Exact & Approx \\
\hline 5 & 0 & 0.93 & 1.0015 & 0.23 & 0.24 & $0.19 \mathrm{E}-2$ & $0.20 \mathrm{E}-2$ \\
\hline 5 & 5 & 0.65 & 0.70 & 0.14 & 0.14 & $0.82 E-3$ & $0.80 E-3$ \\
\hline 5 & 15 & 0.33 & 0.32 & 0.045 & 0.037 & $0.98 E-4$ & $0.40 E-4$ \\
\hline 5 & 25 & 0.19 & 0.16 & 0.016 & 0.009 & $0.91 E-5$ & $0.10 E-5$ \\
\hline 5 & 35 & 0.12 & 0.09 & 0.0061 & 0.0025 & $0.88 \mathrm{E}-6$ & $0.20 \mathrm{E}-7$ \\
\hline 5 & 45 & 0.082 & 0.056 & 0.0025 & 0.0008 & $0.81 E-7$ & $0.50 \mathrm{E}-9$ \\
\hline 15 & 0 & 0.47 & 0.53 & 0.14 & 0.15 & $0.14 \mathrm{E}-2$ & $0.15 E-2$ \\
\hline 15 & 5 & 0.39 & 0.43 & 0.10 & 0.11 & $0.88 \mathrm{E}-3$ & $0.88 E-3$ \\
\hline 15 & 15 & 0.25 & 0.26 & 0.053 & 0.05 & $0.29 \mathrm{E}-3$ & $0.19 E-3$ \\
\hline 15 & 25 & 0.16 & 0.16 & 0.026 & 0.022 & $0.50 \mathrm{E}-4$ & $0.25 E-4$ \\
\hline 15 & 35 & 0.11 & 0.10 & 0.013 & 0.009 & $0.96 E-5$ & $0.27 E-5$ \\
\hline 15 & 45 & 0.08 & 0.07 & 0.0061 & 0.004 & $0.18 E-5$ & $0.27 E-6$ \\
\hline
\end{tabular}




\section{TABLE 4}

\section{APPROXIMATE: FLUX EXACT FiuX}

from A Cylindrtcal Source at Detector. Polnts Outside the Radial and Axial Extenstons of the Source with an Intervening Siab Shield Perpendicisiar to the source Axis

$$
\begin{aligned}
& R=10.00 \quad \text { Mus: } 10 \quad a / R=\quad 20 \quad \text { Muser }=1.00
\end{aligned}
$$

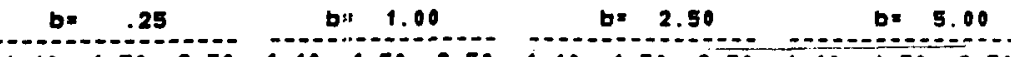

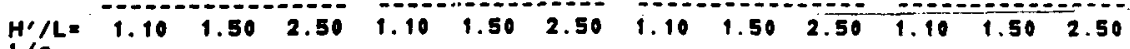

$$
\begin{aligned}
& \begin{array}{rrrrrrrrrrrrr}
1 / 9 \\
8.25 & 1.31 & 1.11 & 1.10 & 1.48 & 1.15 & 1.08 & 1.58 & 1.24 & 1.07 & 1.84 & 1.34 & 1.08 \\
15.83 & .75 & .88 & 1.01 & .83 & .88 & 1.01 & .99 & .88 & 1.00 & 1.15 & .92 & .99
\end{array}
\end{aligned}
$$

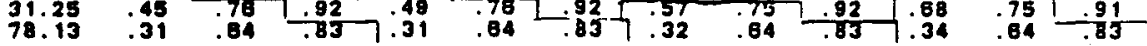

$$
\begin{aligned}
& R=10.00 \quad \text { Mus: } .10 \quad a / R=\quad .50 \quad \text { Mus*R= } 1.00
\end{aligned}
$$

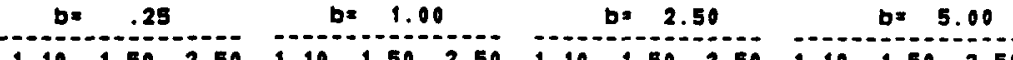

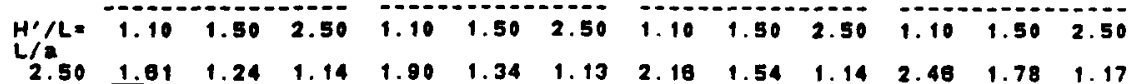

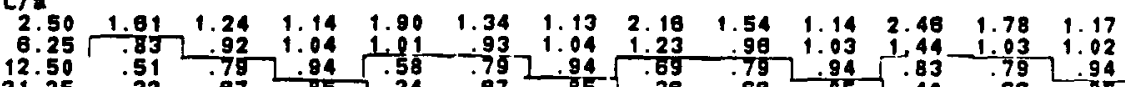

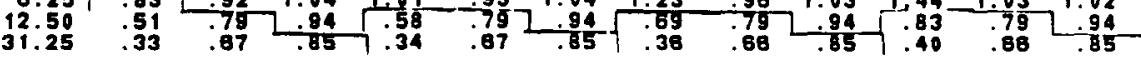

$$
\begin{aligned}
& R=10.00 \quad \text { Mus: } 10 \quad a / R=1.00 \quad \text { Mus }=R=1.00
\end{aligned}
$$

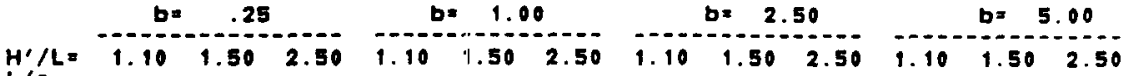

$$
\begin{aligned}
& \begin{array}{llllllllllllll}
L / 8 & 1.25 & 1.92 & 1.42 & 1.22 & 2.41 & 1.64 & 1.22 & 2.97 & 2.05 & 1.28 & 3.72 & 2.60 & 1.38
\end{array} \\
& \begin{array}{lllllllllllll}
3.13 & 1.02 & .99 & 1.08 & 1.27 & 1.01 & 1.07 & 1.57 & 1.08 & 1.07 & 1.89 & 1.23 & 1.06
\end{array}
\end{aligned}
$$

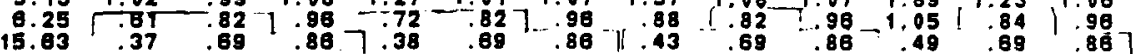

$$
\begin{aligned}
& R=10.00 \quad \text { Mus= } \quad 10 \quad a / R=2.50 \quad \text { Mus }=1.00 \\
& b=.25 \quad b=1.00 \quad b=2.50 \quad b .00
\end{aligned}
$$

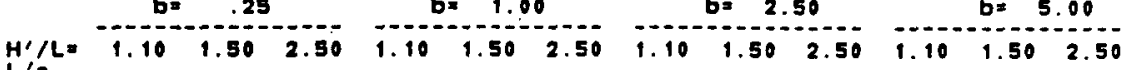

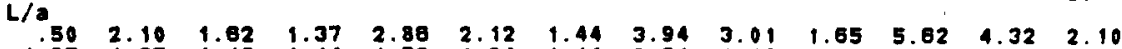

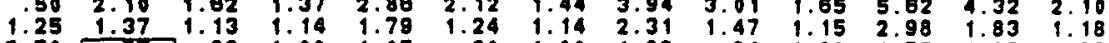

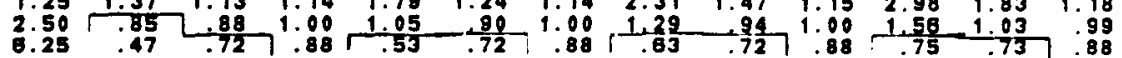

$$
\begin{aligned}
& R=10.00 \quad \text { Mus= } 50 \quad a / R=\quad .20 \quad \text { Mus=R= } 5.00
\end{aligned}
$$

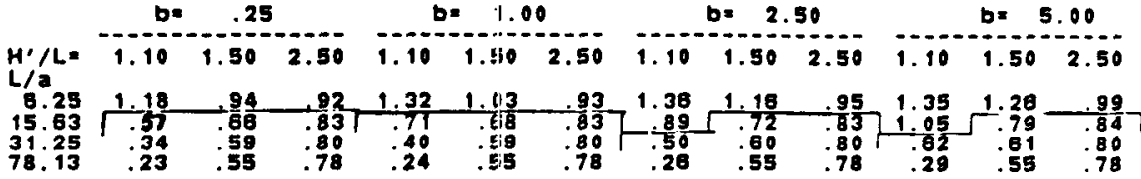

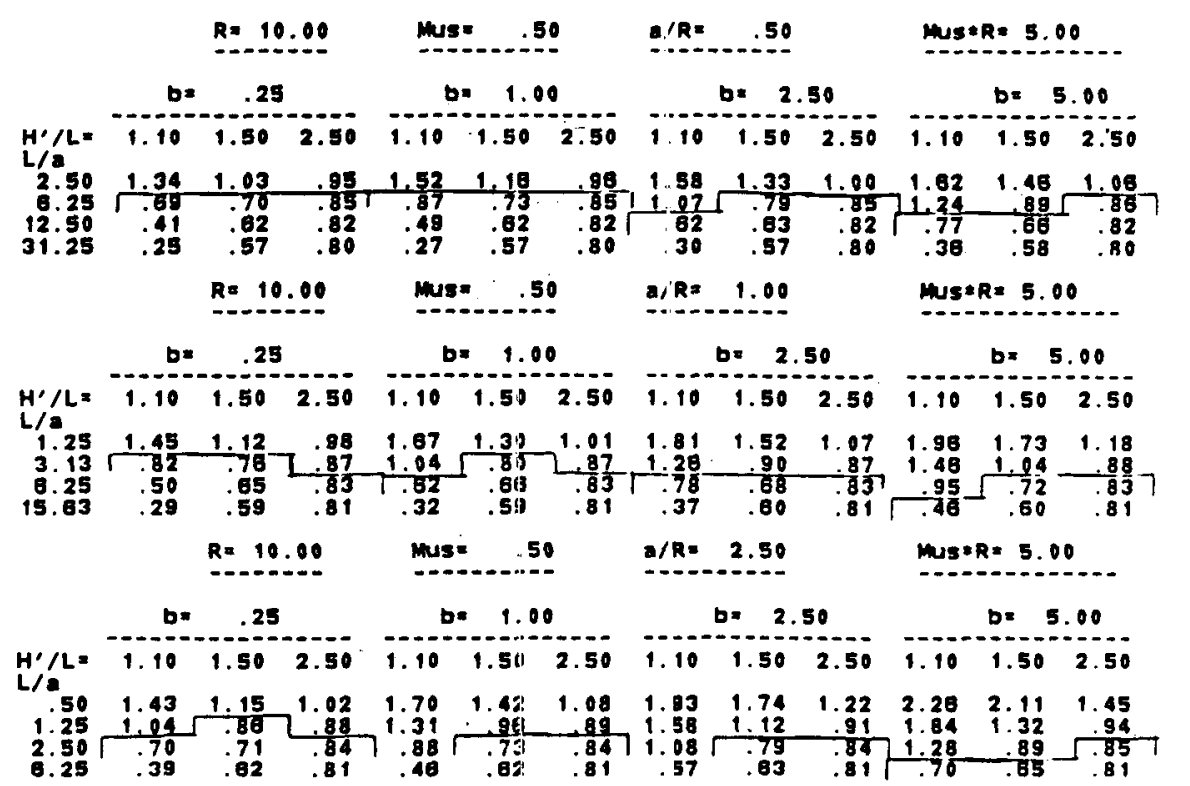


WAPD-TM-1623

TABLE 4 (Cont'd)

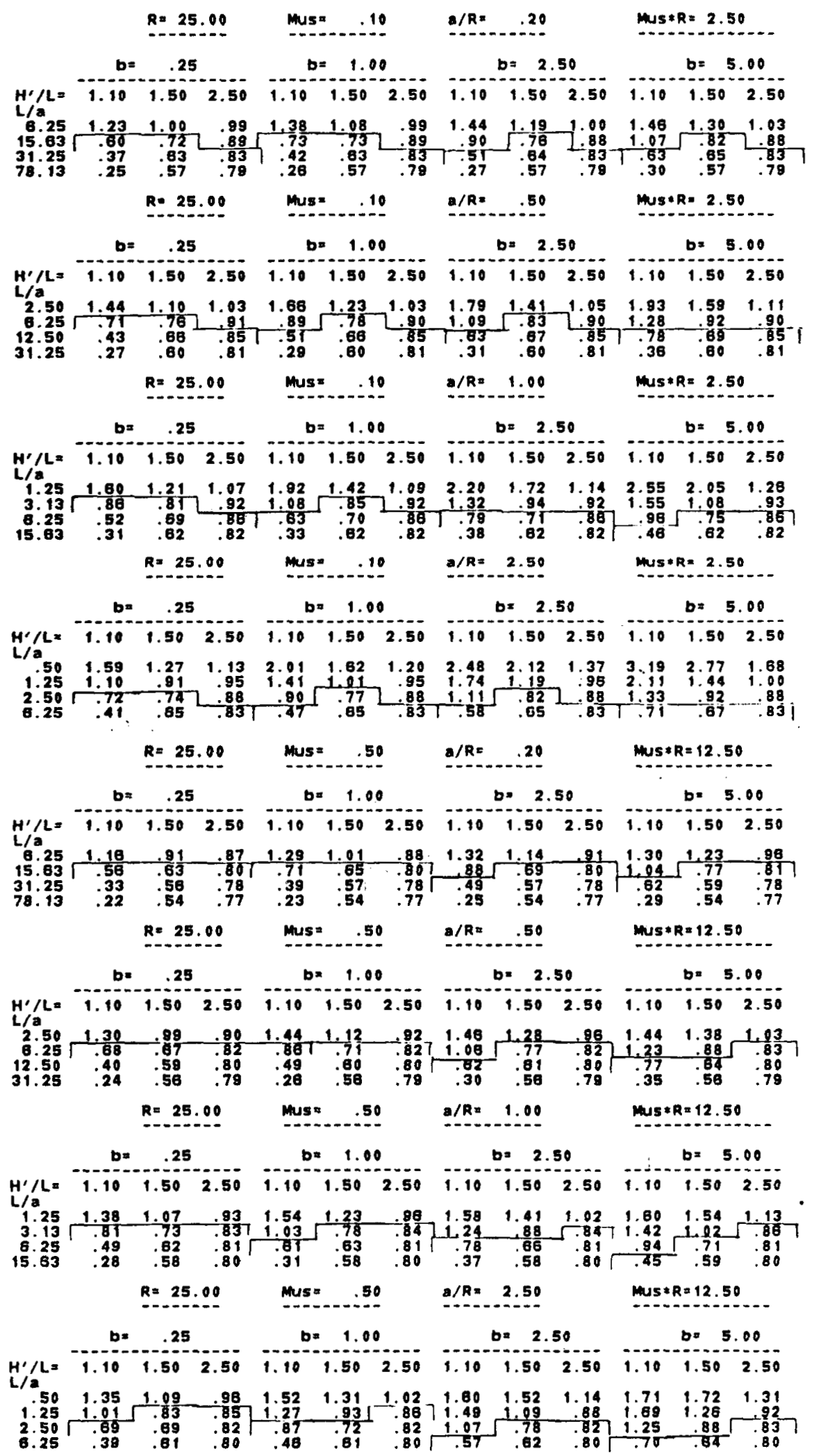




\section{TAELE 5}

Results From the Exact Flux Formula and

From Equation (30) for the Problem of Figure 10

$$
u_{S}=0.1 \quad S_{v}=1.0 \quad R=10 .
$$

\begin{tabular}{|c|c|c|c|c|c|c|c|}
\hline \multirow{3}{*}{ 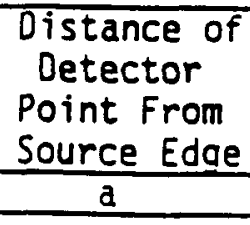 } & \multirow{3}{*}{$\begin{array}{c}\text { Distance of } \\
\text { Detector } \\
\text { Point Below } \\
\text { Source } \\
z \\
\end{array}$} & \multicolumn{6}{|c|}{ Slab Shield Thickness } \\
\hline & & \multicolumn{2}{|c|}{$\frac{b=0.0}{\text { flux }}$} & \multicolumn{2}{|c|}{$\begin{array}{c}b=1.0 \\
F \text { Tux }\end{array}$} & \multicolumn{2}{|c|}{$\frac{b=5.0}{F 7 u x}$} \\
\hline & & Exact & Appr $0 \bar{x}$ & Exact & Approx & Exact & ADDrOX \\
\hline 5 & 0 & 0.93 & NA & 0.23 & NA & $0.20 \mathrm{E}-2$ & NA \\
\hline 5 & 5 & 0.65 & 0.43 & 0.14 & 0.11 & $0.95 E-3$ & $1.1 E-3$ \\
\hline 5 & 15 & 0.33 & 0.27 & 0.098 & 0.08 & $0.92 E-3$ & $0.86 E-3$ \\
\hline 5 & 25 & 0.19 & 0.17 & 0.062 & 0.056 & $0.76 E-3$ & $0.70 E-3$ \\
\hline 5 & 35 & 0.12 & $0.1 \%$ & 0.041 & 0.039 & $0.58 E-3$ & $0.55 E-3$ \\
\hline 5 & 45 & 0.08 & 0.088 & 0.029 & 0.029 & $0.44 E-3$ & $0.43 E-3$ \\
\hline 15 & 0 & 0.47 & NA & 0.14 & NA & $0.14 E-2$ & NA \\
\hline 15 & 5 & 0.39 & 0.33 & 0.066 & 0.077 & $0.31 E-3$ & $0.55 E-3$ \\
\hline 15 & 15 & 0.25 & 0.23 & 0.062 & 0.061 & $0.39 E-3$ & $0.51 E-3$ \\
\hline 15 & 25 & 0.16 & 0.16 & 0.048 & 0.048 & $0.42 E-3$ & $0.46 \mathrm{E}-3$ \\
\hline 15 & 35 & 0.11 & 0.11 & 0.036 & 0.036 & $0.39 E-3$ & $0.41 E-3$ \\
\hline 15 & 45 & 0.08 & 0.08 & 0.027 & 0.027 & $0.33 E-3$ & $0.35 E-3$ \\
\hline
\end{tabular}


TABLE 6

The Function $L_{0}\left(\phi_{0}, b\right)$

\begin{tabular}{|c|c|c|c|c|c|c|c|c|c|c|c|c|c|c|}
\hline & .01 & 05 & $\therefore 10$ & .20 & .40 & .60 & . BO & 1.00 & 1.25 & 1.50 & 1.75 & 2.00 & 2.50 & 3.00 \\
\hline $\begin{array}{r}.01745 \\
.04363 \\
.08727 \\
13090 \\
.17453 \\
.21817 \\
.26180 \\
.34907 \\
.43833 \\
.52360 \\
.61087 \\
.69813 \\
.78540 \\
.87288 \\
1.04720 \\
1.22173 \\
1.39628 \\
1.57080\end{array}$ & $\begin{array}{r}1.00000 \\
1.00000 \\
.99998 \\
.99996 \\
.99992 \\
.99988 \\
.99982 \\
.99988 \\
.99948 \\
.99925 \\
.99895 \\
.99858 \\
.89813 \\
.99758 \\
.99607 \\
.99383 \\
.98988 \\
.98454\end{array}$ & $\begin{array}{r}1.00000 \\
.99998 \\
.99991 \\
.99979 \\
.99962 \\
.99941 \\
.89915 \\
.99848 \\
.89755 \\
.99640 \\
.99498 \\
.99324 \\
.99112 \\
.98856 \\
.98158 \\
.97058 \\
.95051 \\
.89281\end{array}$ & $\begin{array}{l}.99999 \\
.99995 \\
.99982 \\
.99959 \\
.99927 \\
.99886 \\
.99835 \\
.99703 \\
.99528 \\
.99307 \\
.99033 \\
.98701 \\
.98298 \\
.97813 \\
.96511 \\
.94510 \\
.91072 \\
.83771\end{array}$ & $\begin{array}{r}.99999 \\
.99991 \\
.99965 \\
.99922 \\
.99861 \\
.99792 \\
.99684 \\
.99432 \\
.99100 \\
.98681 \\
.98166 \\
.97542 \\
.96794 \\
.95901 \\
.93553 \\
.90108 \\
.84743 \\
.76513\end{array}$ & $\begin{array}{l}99897 \\
99984 \\
99934 \\
99852 \\
99736 \\
99588 \\
99402 \\
98927 \\
98305 \\
97525 \\
96574 \\
95435 \\
94088 \\
92508 \\
88498 \\
83049 \\
75778 \\
67638\end{array}$ & $\begin{array}{l}99998 \\
99976 \\
99905 \\
.99785 \\
.99817 \\
99400 \\
.99134 \\
.98451 \\
.97558 \\
96447 \\
95102 \\
.93508 \\
91645 \\
.89491 \\
.84208 \\
.77487 \\
69521 \\
61874\end{array}$ & $\begin{array}{l}89993 \\
99969 \\
99876 \\
99780 \\
99502 \\
.9921 \\
89876 \\
87992 \\
86844 \\
95421 \\
93713 \\
91708 \\
.89391 \\
.86751 \\
80468 \\
.72932 \\
84797 \\
.57621\end{array}$ & $\begin{array}{l}.99994 \\
99982 \\
99848 \\
99857 \\
99390 \\
99046 \\
98624 \\
97547 \\
96153 \\
94436 \\
92390 \\
90009 \\
87289 \\
84232 \\
77158 \\
.69111 \\
61046 \\
.54271\end{array}$ & $\begin{array}{l}98993 \\
.99953 \\
.99813 \\
99579 \\
99252 \\
98831 \\
.98318 \\
.97004 \\
95316 \\
.93251 \\
.90811 \\
.88004 \\
.84839 \\
.81341 \\
.73507 \\
.65106 \\
.57288 \\
.50907\end{array}$ & $\begin{array}{l}98991 \\
99945 \\
99779 \\
99503 \\
99118 \\
98620 \\
98014 \\
98474 \\
94503 \\
92109 \\
89305 \\
88112 \\
82559 \\
.78693 \\
.70295 \\
81746 \\
54189 \\
48168\end{array}$ & $\begin{array}{l}99990 \\
99936 \\
99745 \\
99427 \\
98983 \\
98412 \\
97716 \\
95955 \\
93712 \\
91005 \\
87862 \\
84319 \\
.80427 \\
.76253 \\
.67445 \\
58878 \\
.51608 \\
45872\end{array}$ & $\begin{array}{l}.9988 \\
.99928 \\
99712 \\
.99352 \\
98850 \\
.98206 \\
97423 \\
95445 \\
.92939 \\
89935 \\
.86475 \\
82815 \\
.78425 \\
.73997 \\
64895 \\
.58393 \\
49393 \\
.43905\end{array}$ & $\begin{array}{l}99988 \\
.99911 \\
99648 \\
99204 \\
98589 \\
97802 \\
96847 \\
94450 \\
91443 \\
97885 \\
83853 \\
.79441 \\
74764 \\
.69953 \\
.60524 \\
52281 \\
.45781 \\
.40677\end{array}$ & $\begin{array}{l}99983 \\
998985 \\
99580 \\
99958 \\
98331 \\
97403 \\
96281 \\
93482 \\
99005 \\
85942 \\
81410 \\
78542 \\
71493 \\
66429 \\
.56907 \\
48992 \\
42873 \\
.38110\end{array}$ \\
\hline (MFP) & 3.50 & 4.00 & 5.00 & 6.00 & 8.00 & 10.00 & $12: 00$ & 15.00 & 20.00 & 25.00 & 35.00 & 50.00 & 60.00 & 7500 \\
\hline $\begin{array}{r}.01745 \\
.04383 \\
.08727 \\
.13096 \\
.17453 \\
.21817 \\
.28180 \\
.34907 \\
.43833 \\
.52360 \\
.61087 \\
.69813 \\
.78540 \\
.87268 \\
1.04720 \\
1.22173 \\
1.39628 \\
1.57080\end{array}$ & $\begin{array}{l}99981 \\
.99879 \\
99515 \\
98913 \\
98078 \\
97010 \\
95728 \\
.92538 \\
88618 \\
84095 \\
79123 \\
73880 \\
68553 \\
68333 \\
.53859 \\
.46281 \\
40499 \\
.35998\end{array}$ & $\begin{array}{l}99978 \\
.99862 \\
99451 \\
98769 \\
97823 \\
96622 \\
95178 \\
91618 \\
87279 \\
82334 \\
76978 \\
.71427 \\
.65897 \\
60590 \\
51251 \\
43992 \\
.38494 \\
.34217\end{array}$ & $\begin{array}{l}99973 \\
.99830 \\
.99322 \\
98483 \\
.97324 \\
95859 \\
.94107 \\
.89838 \\
.84729 \\
.79044 \\
.73081 \\
.87058 \\
61288 \\
.55952 \\
.47004 \\
.40308 \\
.35270 \\
.31351\end{array}$ & $\begin{array}{r}99968 \\
.99798 \\
99193 \\
.98201 \\
.98831 \\
.95109 \\
.93062 \\
88127 \\
.82334 \\
.78031 \\
.69573 \\
.83287 \\
.57432 \\
.52180 \\
.43674 \\
.37441 \\
.32781 \\
.20121\end{array}$ & $\begin{array}{l}99957 \\
99734 \\
98942 \\
97642 \\
95863 \\
93649 \\
91048 \\
84906 \\
77951 \\
70708 \\
63645 \\
57124 \\
51357 \\
48412 \\
38727 \\
33195 \\
29046 \\
25818\end{array}$ & $\begin{array}{l}99947 \\
99870 \\
.98691 \\
97092 \\
94919 \\
92235 \\
89117 \\
81918 \\
74041 \\
.68161 \\
38812 \\
52317 \\
48792 \\
42187 \\
35170 \\
30148 \\
.26378 \\
.23447\end{array}$ & $\begin{array}{l}99937 \\
99807 \\
98442 \\
98548 \\
93992 \\
90864 \\
87269 \\
79141 \\
70535 \\
62245 \\
54810 \\
48473 \\
43230 \\
38938 \\
32451 \\
27815 \\
24338 \\
21634\end{array}$ & $\begin{array}{l}99922 \\
.99512 \\
98071 \\
95744 \\
92635 \\
88880 \\
84835 \\
75325 \\
65917 \\
57310 \\
49967 \\
.43981 \\
39127 \\
.35222 \\
29352 \\
25159 \\
22014 \\
19568\end{array}$ & $\begin{array}{l}99896 \\
.99355 \\
.97459 \\
.04432 \\
90456 \\
.85757 \\
.80584 \\
69787 \\
59590 \\
509938 \\
.44002 \\
.38569 \\
34293 \\
.30865 \\
.25721 \\
22046 \\
199290 \\
17147\end{array}$ & $\begin{array}{l}99871 \\
.99198 \\
96685 \\
93155 \\
88374 \\
82844 \\
76909 \\
65040 \\
54583 \\
48159 \\
.39704 \\
34781 \\
30900 \\
.27810 \\
.23775 \\
19865 \\
17382 \\
15450\end{array}$ & $\begin{array}{l}99820 \\
.98885 \\
.95687 \\
.80698 \\
84477 \\
.77575 \\
70518 \\
57492 \\
.47139 \\
39487 \\
33870 \\
29838 \\
.26345 \\
.23710 \\
19759 \\
18938 \\
14819 \\
13172\end{array}$ & 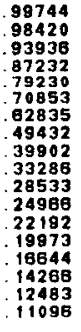 & $\begin{array}{l}99694 \\
98113 \\
92814 \\
85081 \\
78084 \\
.67023 \\
58687 \\
.45481 \\
36551 \\
.30470 \\
26117 \\
.22852 \\
.20313 \\
18282 \\
15235 \\
13059 \\
111426 \\
10157\end{array}$ & $\begin{array}{r}99618 \\
.97654 \\
.91177 \\
.81986 \\
.71822 \\
.62070 \\
.53582 \\
.40922 \\
.32791 \\
27328 \\
.23424 \\
.20496 \\
.18219 \\
.16397 \\
.13664 \\
.11712 \\
.10248 \\
09109\end{array}$ \\
\hline
\end{tabular}


TABI.E 7

The Function $G\left(\theta_{0}, b\right)$

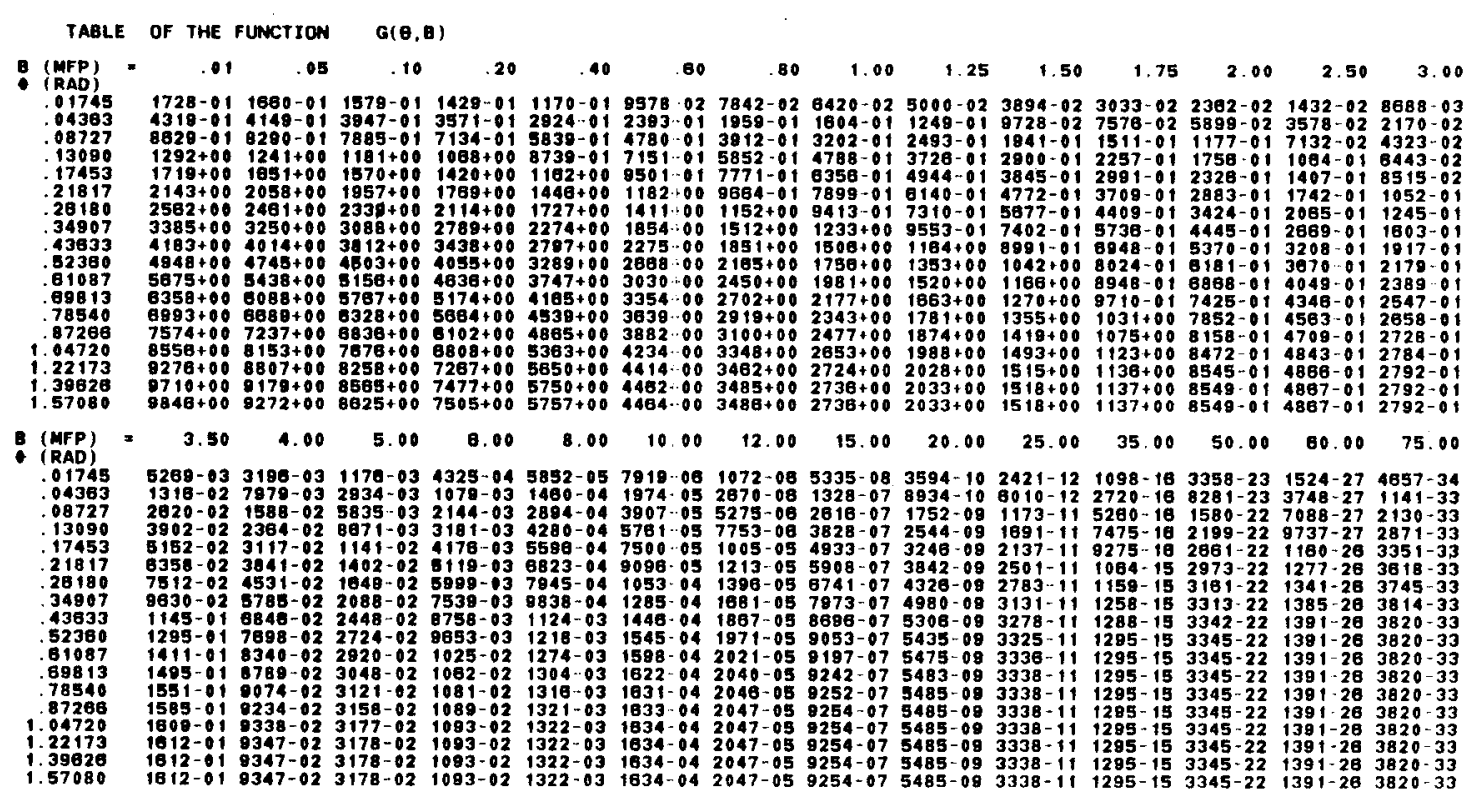

TABLE 8

The Smoothed Function $\bar{G}\left(\theta_{0}, b\right)=G\left(\theta_{0}, b\right) e^{b} / \theta_{0}$

TABLE OF THE SMOOTHED FUNCTION $\operatorname{EXP}(B)=G(\theta, B) / \theta$

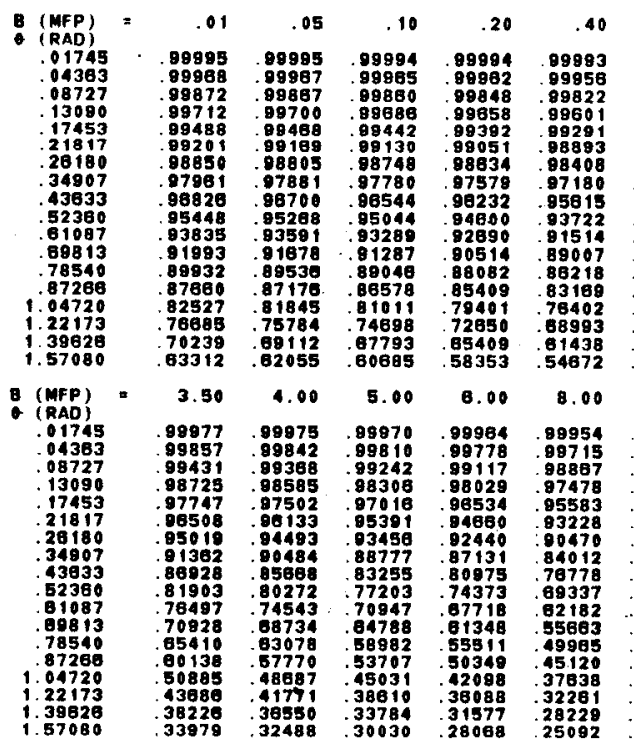

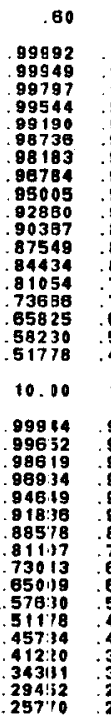

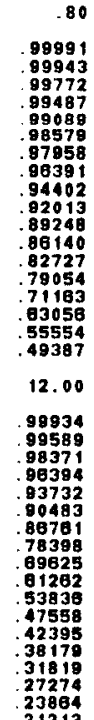

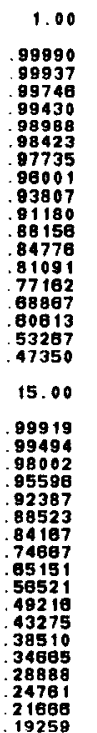

\begin{tabular}{|c|c|}
\hline 1.25 & 1.50 \\
\hline $\begin{array}{l}99989 \\
99929 \\
99715 \\
99359 \\
98983 \\
98228 \\
97457 \\
95517 \\
93072 \\
90160 \\
86828 \\
83133 \\
79143 \\
74937 \\
66253 \\
57933 \\
50817 \\
45171\end{array}$ & $\begin{array}{l}99987 \\
99991 \\
99883 \\
99288 \\
98738 \\
98034 \\
97181 \\
85038 \\
92349 \\
89162 \\
85540 \\
8555 \\
77294 \\
72855 \\
63888 \\
55591 \\
48712 \\
43299\end{array}$ \\
\hline 20.00 & 25.00 \\
\hline $\begin{array}{l}99893 \\
99337 \\
97393 \\
84291 \\
90225 \\
85431 \\
80199 \\
69222 \\
58998 \\
50364 \\
43482 \\
38107 \\
33881 \\
30493 \\
2541 \\
21781 \\
19058 \\
16941\end{array}$ & $\begin{array}{l}99888 \\
99181 \\
98790 \\
93018 \\
88156 \\
82544 \\
78337 \\
64581 \\
54093 \\
45728 \\
39322 \\
34424 \\
30601 \\
27541 \\
22950 \\
19672 \\
17213 \\
15300\end{array}$ \\
\hline
\end{tabular}

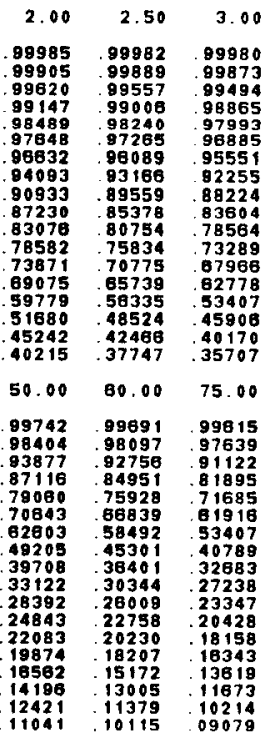


WAPD-TM-1623

TABLE 9

The Smoothed Function $\bar{F}\left(\theta_{0}, b\right)=F\left(\theta_{0}, b\right) e^{b} / \theta_{0}$

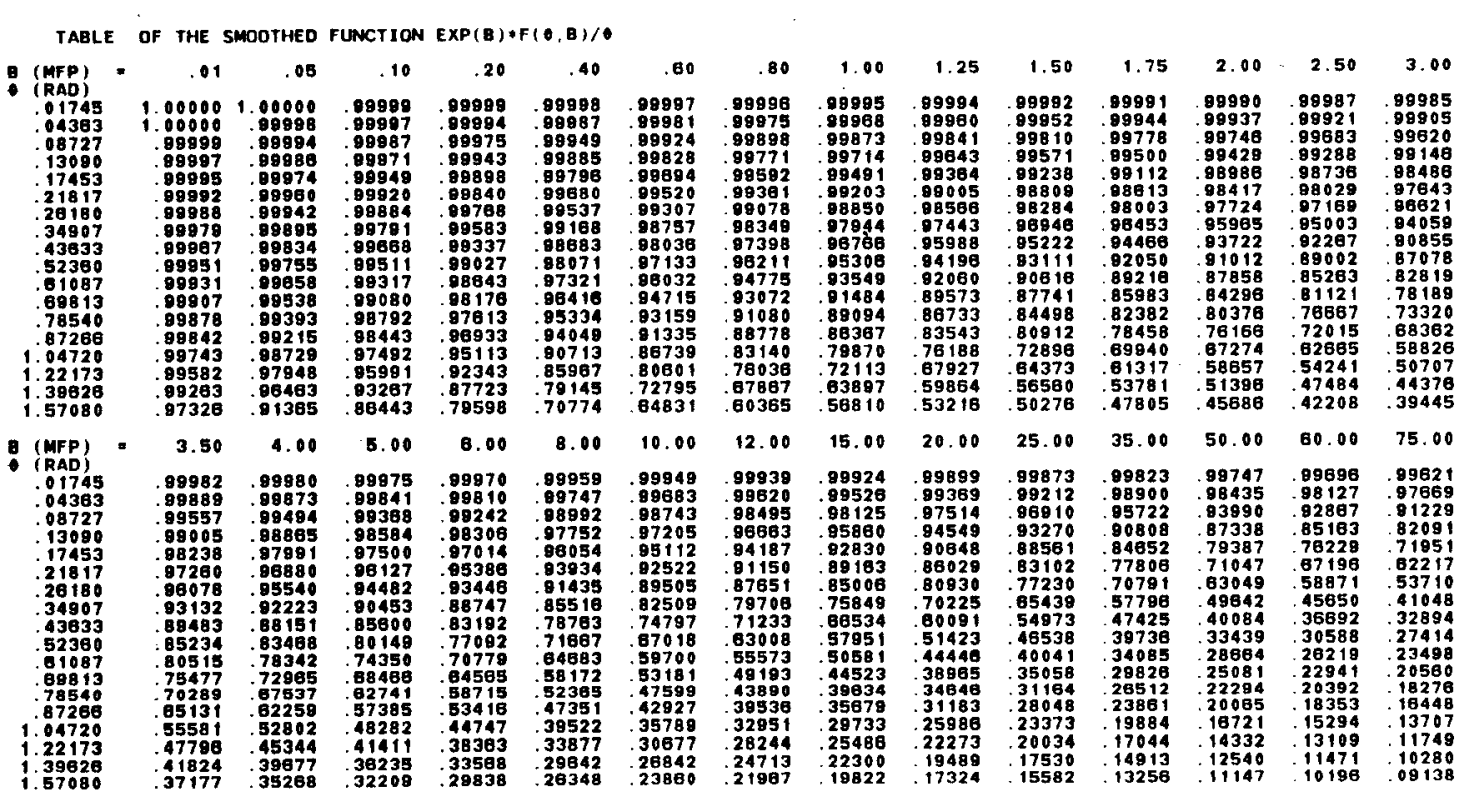

TABLE 10

The Function $F\left(\theta_{0}, b\right)$

TABLE OF THE FUNCTION $F(\bullet, B)$

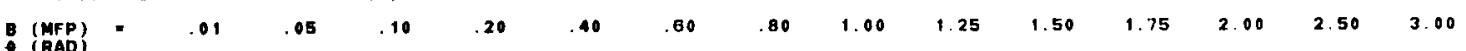

RAD $1745 \quad 1728-01 \quad 4860-01 \quad 1579-01 \quad 1429-01 \quad 1170-019578-02 \quad 7842-028420-02 \quad 5000-02 \quad 3894-02$ 3033-02 2382-02 1432-02 8688-03

04383 4320-01 4150-01 3948-01 3572-01 2924-01 2394-01 1960-01 1605-01 1250-01 9731-02 7578-02 5901-02 3579-02 2170-02

8640-01 8301-01 7895-of 7143-01 5847-01 4786-01 3917-01 3206-01 2498-01 1943-01 1513-01 1178-01 7141-02 4328-02

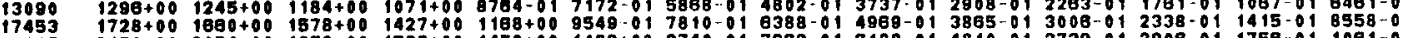

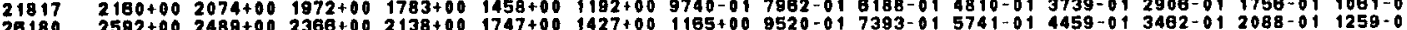

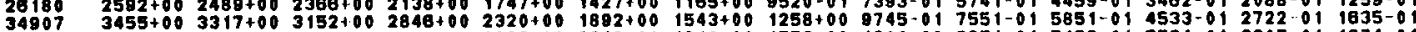

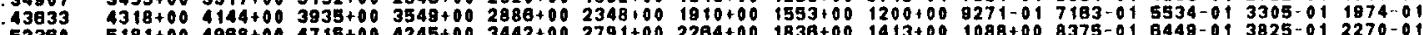

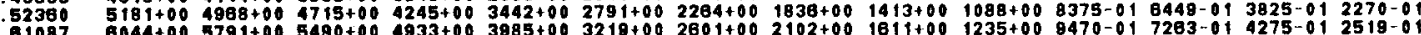

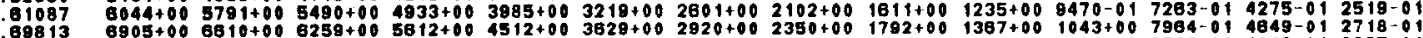

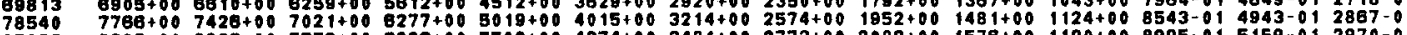

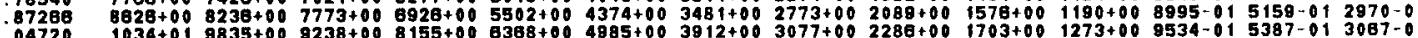

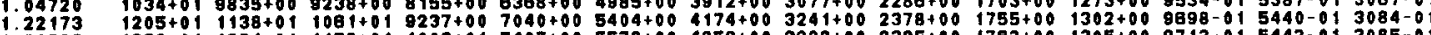

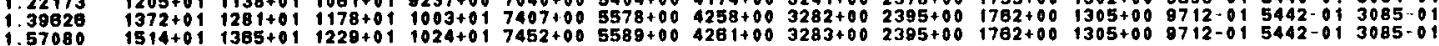

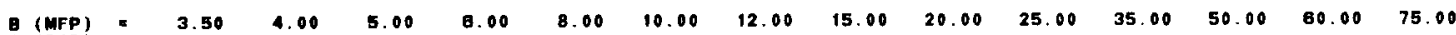

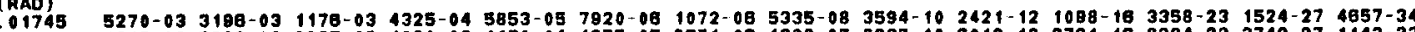

.04363 1316-02 7982-03 2935-03 1080-03 1460-04 1975-05 2871-06 1328-07 8937-10 6012-12 2721-16 8284-23 3748-27 1142-33

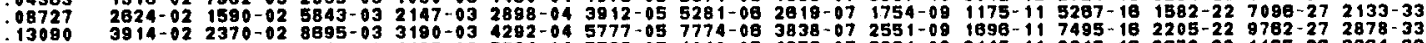

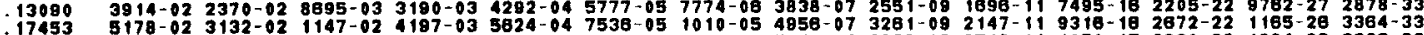

21817 6408-02 3871-02 1413-02 5158-03 6875-04 9164-05 1222-05 5950-07 3868-09 2518-11 1070-15 2980-22 1284-28 3638-33

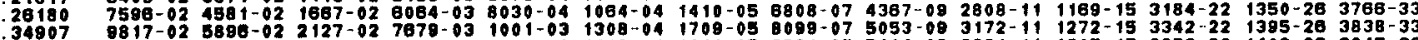

$.43933 \quad 1179-01$ 7048-02 2517-02 8998-03 1153-03 1482-04 1910-05 8889-07 8404-09 3331-11 1305-15 3373-22 1402-28 3845-33

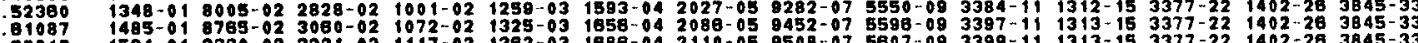

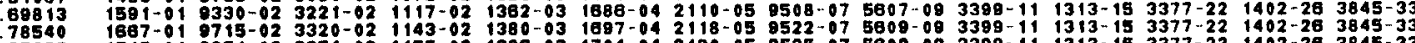

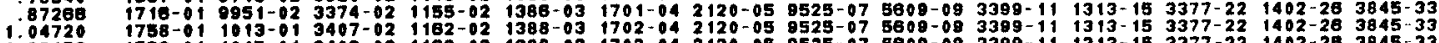

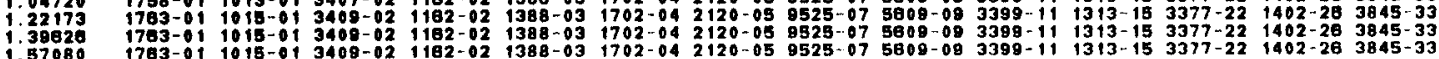


TABLE 11

The Smoothed Function $\bar{F}_{2}\left(\theta_{c}, b\right)=F_{2}\left(\theta_{0}, b\right) e^{b} / \theta_{0}$

\begin{tabular}{|c|c|c|c|c|c|c|c|c|c|c|c|c|c|c|}
\hline & & .08 & .10 & .20 & .40 & .60 & .80 & S. & 1.25 & 1.50 & 1.75 & 2.00 & 2.50 & 3.00 \\
\hline 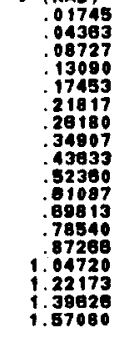 & $\begin{array}{r}1.032 \\
1.048 \\
1.097 \\
1.090 \\
1.150 \\
1.253 \\
1.412 \\
1.721 \\
3.018\end{array}$ & $\begin{array}{l}0 \\
0 \\
0 \\
0 \\
0 \\
0 \\
0 \\
1 \\
1 \\
2\end{array}$ & $\begin{array}{l}1: 0 \\
180 \\
180 \\
180 \\
1: 0 \\
180 \\
1: 0 \\
1: 2\end{array}$ & 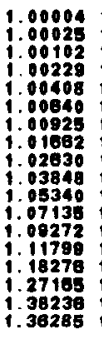 & $\begin{array}{l}812 \\
010 \\
028 \\
038 \\
038 \\
085 \\
088 \\
080 \\
116 \\
151 \\
155 \\
056\end{array}$ & 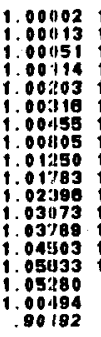 & 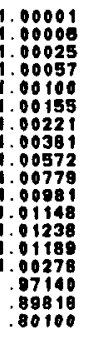 & 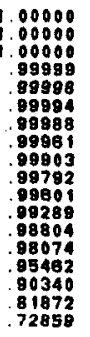 & 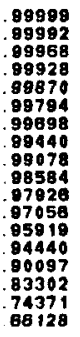 & 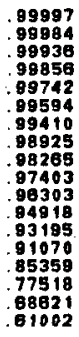 & & & & $\begin{array}{l}748 \\
429\end{array}$ \\
\hline & 3 & 4. & s. & B. & 8 & 10.8 & 12. & 15. & 20.00 & 25.00 & 5.00 & 50.00 & 30. & .0 \\
\hline 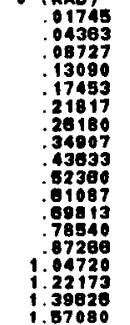 & 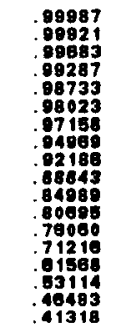 & 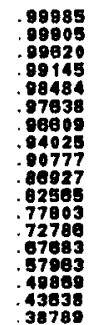 & 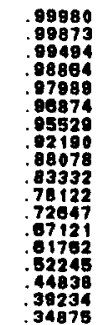 & 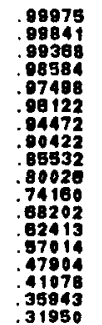 & 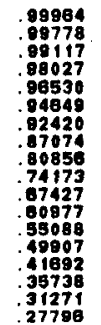 & $\begin{array}{c}67 \\
77 \\
79 \\
18 \\
81 \\
80 \\
75 \\
73 \\
59 \\
59 \\
89 \\
84 \\
94 \\
98\end{array}$ & 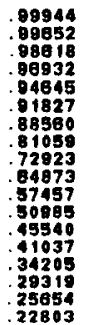 & $\begin{array}{l}8 \\
88 \\
85 \\
77 \\
58 \\
52 \\
74 \\
46 \\
36\end{array}$ & & & & & & 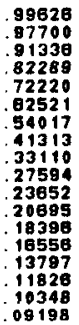 \\
\hline
\end{tabular}

TABLE 12

The Function $F_{2}\left(\theta_{0}, b\right)$

TABLE OF THE FUNCTION F2(0,8)

\begin{tabular}{l} 
(MFP) $=$ \\
\hline (RAD
\end{tabular}

. 01798 1728-01 1800-01 1579-01 1429-01 1170-01 9578-02 7842-02 (1421-02 5000-02 3894-02 3033-02 2382-02 1433-02 8889-03

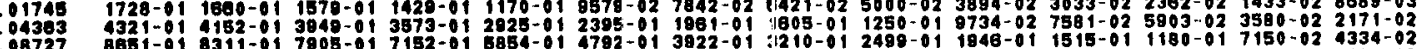

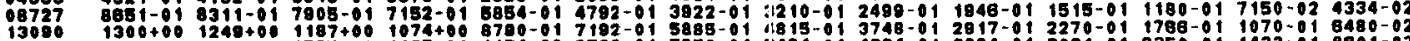

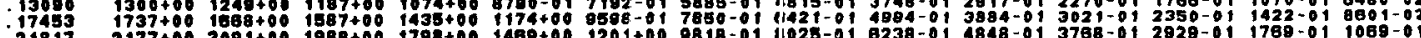

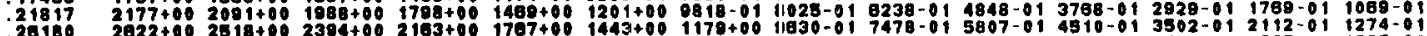

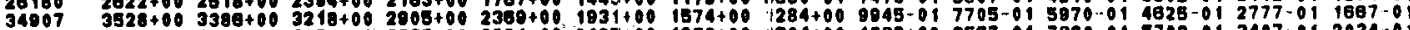

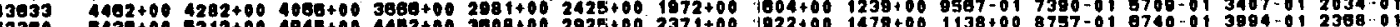

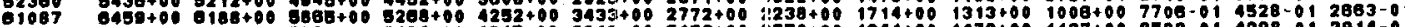

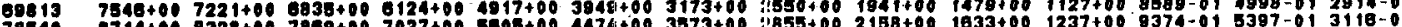

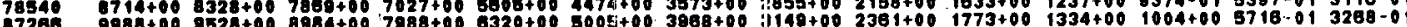

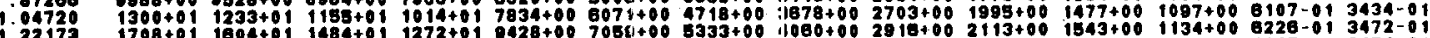

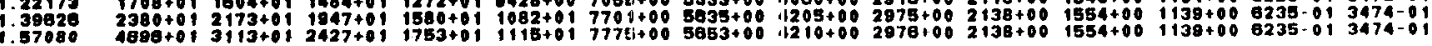

(MFP) $=3.80 \quad 4.00 \quad 8.00 \quad 0.00 \quad 8.00 \quad 10.00 \quad 12.00 \quad 15.00 \quad 20.00 \quad 25.00 \quad 35.00 \quad 50.00 \quad 60.00 \quad 75.00$

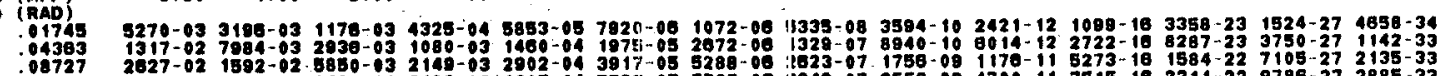

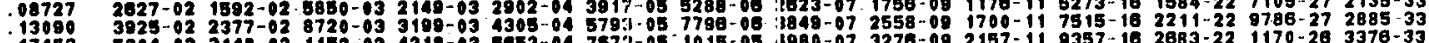

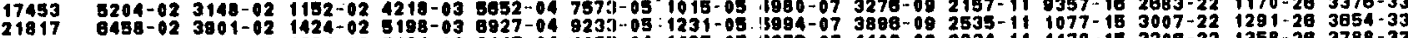

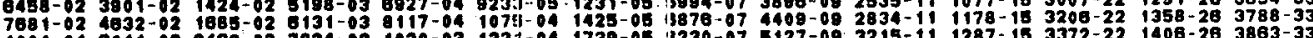

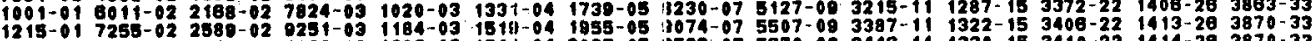
1215-01 7255-02 2580-02 9251-03 194-03 1644-04 2087-05 \$528-07 5670-09 3446-11 1330-15 3410-22 1414-28 $3870-33$

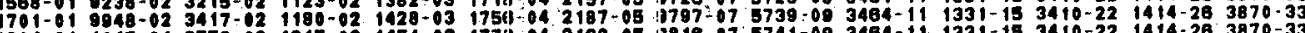
$1804-01$ 1047-01 3552-02 1215-02 145/-03 1772-04 2198-05 3816-07:5744-09 3484-11 1331-15 3410-22 1414-26 $3870-33$

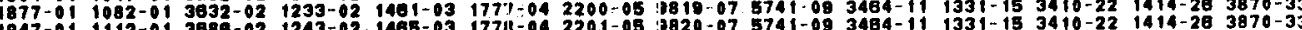
1947-01 1112-01 3886-02 1243-02 1458-03 7771-04 2201-05 3920-67 541-09 3484-11 1331-15 3410-22 1414-26 $3970-33$

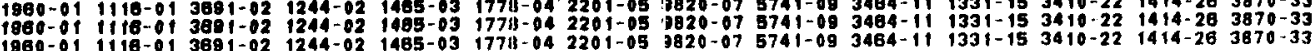


TABLE 13

The Smoothed Function $\bar{R}\left(\phi_{0}, l_{2} / a, b\right)=R\left(\phi_{0}, l_{2} / a, b\right) e^{b} / \phi_{0}$

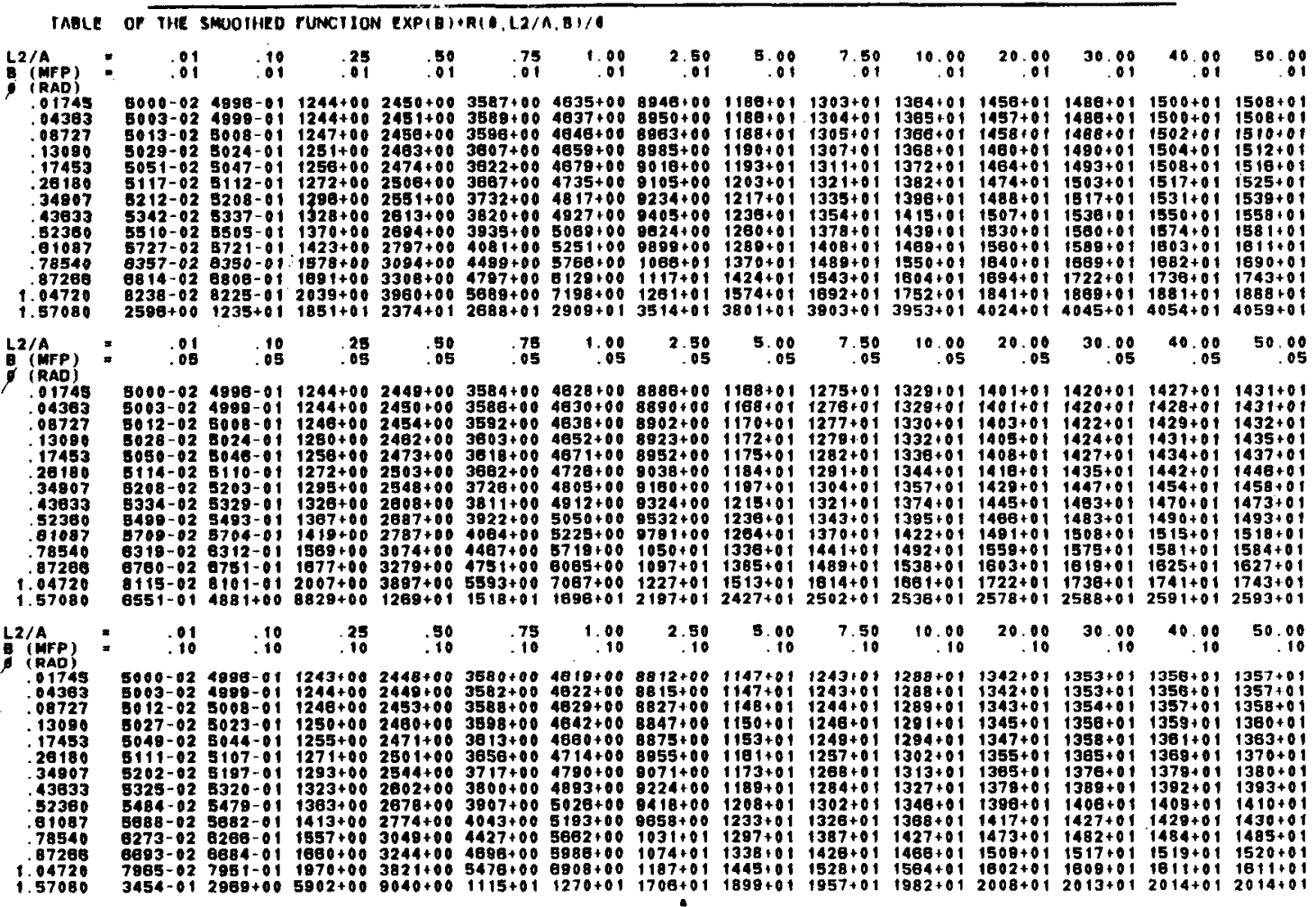

TABLE OF THE SMDOIHED FUNCTION EXP(B)+R1, $, 12 / A, B) / \%$

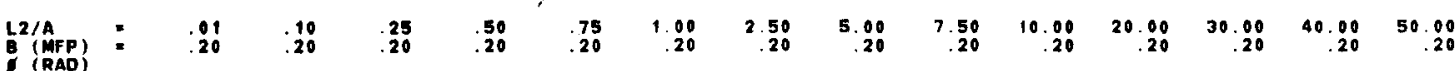

.1745 5000-02 4998-01 1243+00 2445+00 3572+00 4602+00 8668+00 1108+01 1184+01 1215+01 1248+01 1250+01 $1250+01 \quad 1250+01$

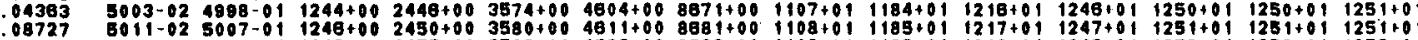
.13090 828-02 5021-01 1249+00 2457+00 3589+00 4823+00 8699+00 1109+01 1188+0I 1218+01 1248+01 $1252+01 \quad 1252+01 \quad 125310$

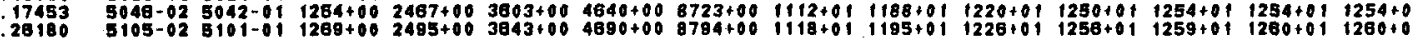

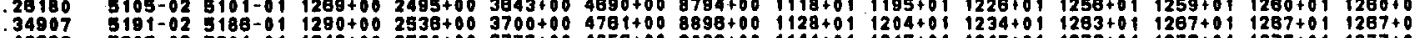

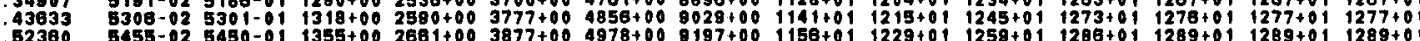

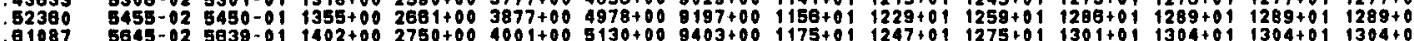
.78540

87280
1.04720
57080

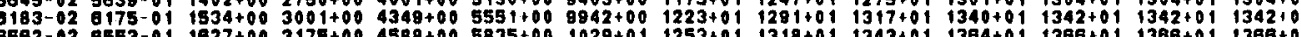

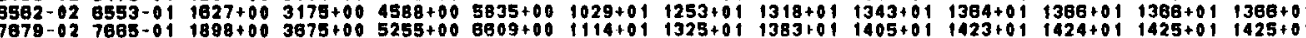
$1855-01 \quad 1738+003771+006177+00 \quad 7882+00 \quad 9158+00 \quad 1277+01 \quad 1428+011465+01 \quad 1480+011492+011493+011493+011493+01$

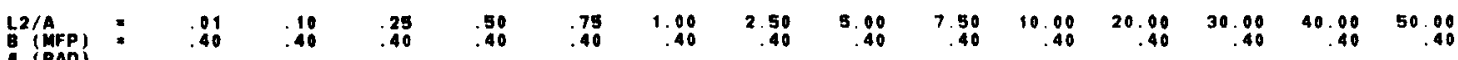
(RAO) $8000-02 \quad 4995-01 \quad 1242+00 \quad 2440+00 \quad 3850+00 \quad 4588100 \quad 8393+00 \quad 1035+01 \quad 1085+01 \quad 1101+01 \quad 1111+01 \quad 1112+011112+011112+01$

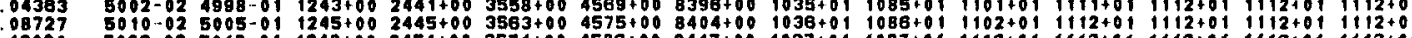
$130905023-025018-01 \quad 1248+002451+003571+00 \quad 4588+008417+001037+01 \quad 1087+011103+011113+011113+011113+011113+0$

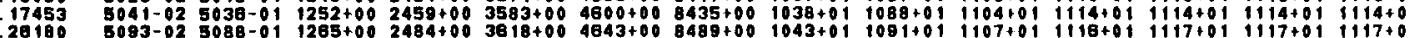

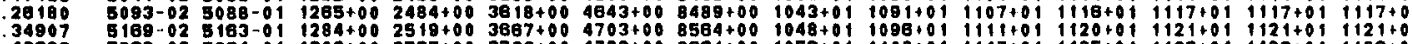

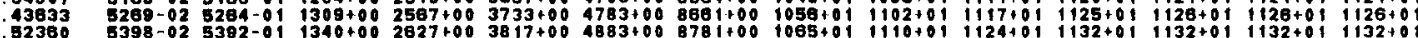
.61087

.78540 $5560-025554-01 \quad 1380+002702+003921+005008+00 \quad 9924+00 \quad 1075+011118+01 \quad 1131+011138+011139+011139+011139+0$ (15)

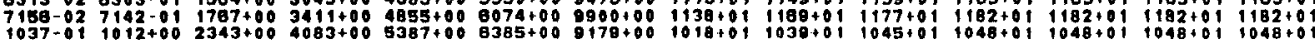

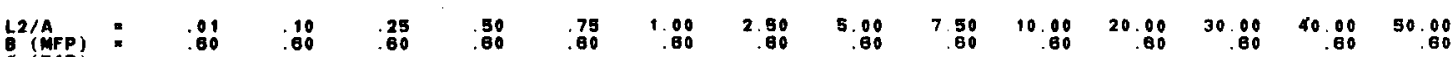
0.01735 .08727 .26160 43833
82300 .82300

.78540

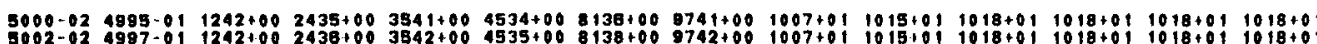

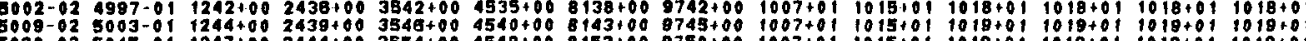

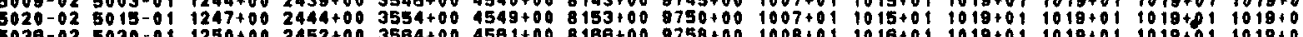

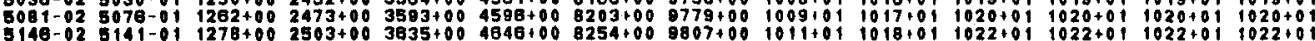
$5233-025227-01 \quad 1299+002543+003889+00 \quad 4711+008318+008842+001013+01 \quad 1020+011023+011023+011023+01102310$ 5343-02 5338-01 1328+00 2594+00 3758+00 4792+00 8397100 9881+00 $1018+0110221011025+011025+011025+011025+0$

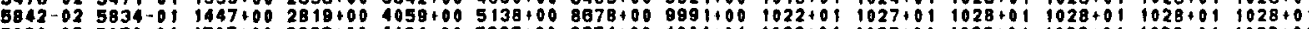

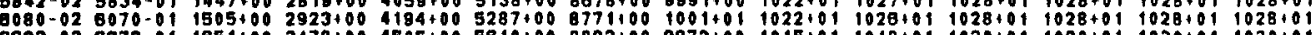

1.04720
1.57080

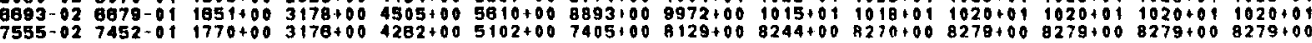


TABLE 13 (Cont'd)

TABLE OF THE SMOOTHED FUNCTION EXP(B)*R(\$,L2/A,B)/O

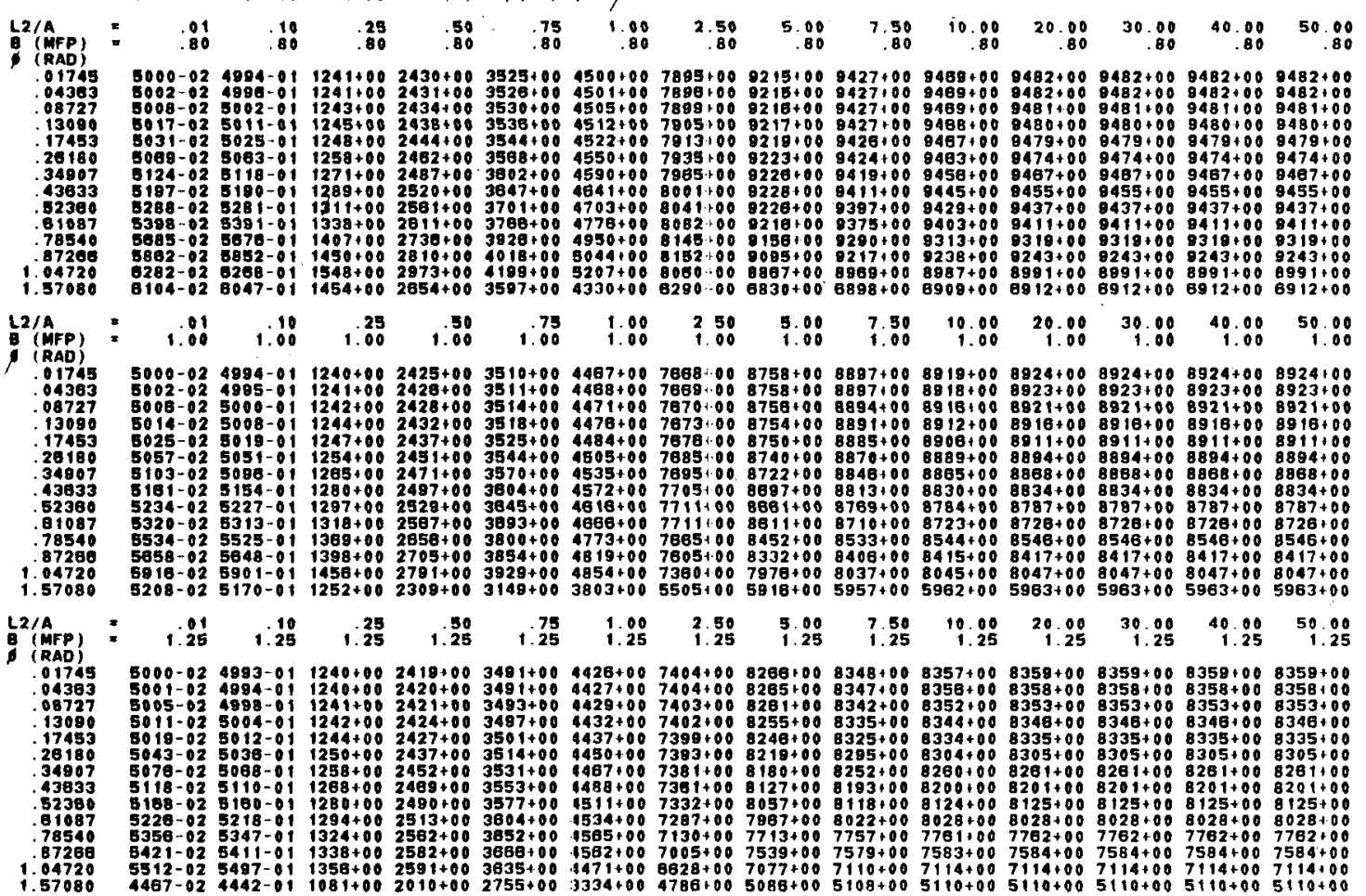

TABLE OF THE SMOOTHED FUNCTION EXP(B)+R(O,L2/A, B 1/6

\begin{tabular}{|c|c|c|c|c|c|c|c|c|c|c|c|c|c|c|}
\hline AfP & $\begin{array}{r}01 \\
1.50\end{array}$ & $\begin{array}{r}10 \\
1.50\end{array}$ & $\begin{array}{r}.25 \\
1.50\end{array}$ & $\begin{array}{r}80 \\
1.50\end{array}$ & $\begin{array}{r}.75 \\
1.50\end{array}$ & $\begin{array}{l}1.00 \\
1.50\end{array}$ & $\begin{array}{l}2.50 \\
1.50\end{array}$ & .00 & $\begin{array}{l}7.50 \\
1.50\end{array}$ & 1. & $\begin{array}{r}20.00 \\
1.50\end{array}$ & 00 & 500 & 0.00 \\
\hline $\begin{array}{r}.04363 \\
.08727 \\
.13090 \\
.17453 \\
.28180 \\
.34907 \\
.43833 \\
.52360 \\
.61087 \\
.78540 \\
.87268 \\
.04720 \\
1.07080\end{array}$ & $\begin{array}{l}5000-02 \\
5001-02 \\
5003-02 \\
5007-02 \\
5013-02 \\
5028-02 \\
8049-02 \\
5074-02 \\
5104-02 \\
3135-02 \\
5189-02 \\
5203-02 \\
5159-02\end{array}$ & $\begin{array}{l}0-01 \\
5-01 \\
0-01 \\
1-01 \\
7-01 \\
5-01 \\
0-01 \\
9-01 \\
1-01 \\
9-01 \\
7-01\end{array}$ & & $\begin{array}{l}2432+00 \\
2442+00 \\
2452+00 \\
2462+00 \\
2474+00 \\
2470+00 \\
2418+60 \\
1790+00\end{array}$ & & $\begin{array}{l}438 \theta+00 \\
4387+00 \\
4387+00 \\
4389+00 \\
4391+00 \\
4398+00 \\
4402+00 \\
4407+00 \\
4410+00 \\
4408+00 \\
4373+00 \\
4327+00 \\
4143+00 \\
2989+00\end{array}$ & & & & & & & & \\
\hline & 1.75 & 1.75 & 1.75 & $\begin{array}{r}.50 \\
1.75\end{array}$ & $\begin{array}{r}1.75 \\
1.75\end{array}$ & & & & 7.50 & $\begin{array}{r}10.00 \\
1.75\end{array}$ & 20.000 & & & \\
\hline $\begin{array}{r}.01745 \\
.64363 \\
.08727 \\
.13090 \\
.17453 \\
.26180 \\
.34907 \\
.43633 \\
.52380 \\
.61087 \\
.78540 \\
.87268 \\
1.04720 \\
1.570080\end{array}$ & $\begin{array}{l}5000-02 \\
5000-02 \\
5001-02 \\
5003-02 \\
5000-02 \\
8013-02 \\
5022-02\end{array}$ & $4982-01$ & $\begin{array}{l}1238+00 \\
1238+00 \\
1236+00 \\
1239+00 \\
1238+00 \\
1241+00 \\
1243+00 \\
1245+00 \\
1246+00 \\
1247+00 \\
1241+00 \\
1232+00 \\
1191+00\end{array}$ & $\begin{array}{l}3 \\
3 \\
3 \\
3 \\
3 \\
3 \\
3 \\
3 \\
3\end{array}$ & & & & & & & & & & \\
\hline A & .01 & $2: 10$ & $2: 28$ & .50 & 2.75 & & & & & & & & & \\
\hline $\begin{array}{r}.01745 \\
.44363 \\
.08727 \\
.13090 \\
.17483 \\
.28190 \\
.34907 \\
.43633 \\
.52360 \\
.61087 \\
.78540 \\
.87286 \\
1.04720\end{array}$ & $\begin{array}{l}50 \\
50 \\
50 \\
50 \\
50 \\
49 \\
49 \\
49 \\
49 \\
49 \\
48 \\
48\end{array}$ & & $\begin{array}{l}12 \\
12 \\
12 \\
12 \\
12 \\
12 \\
11 \\
80\end{array}$ & $\begin{array}{l}2 \\
2 \\
2\end{array}$ & 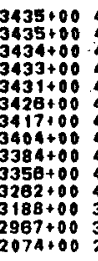 & $\begin{array}{l}4 \\
4 \\
4 \\
4 \\
4 \\
4 \\
4\end{array}$ & & & & & & & & \\
\hline
\end{tabular}




\section{TABLE 13 (Cont'd)}

TABLE of INE SWDOTHED FUNCIION EXP(B)+RIY,L2/A, BI,

\begin{tabular}{|c|c|c|c|c|c|c|c|c|c|c|c|c|c|c|}
\hline 2/A & 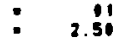 & 2.3 & 2. & 2.3 & 2.50 & & & 2.50 & & 2.50 & 250 & $\begin{array}{l}30.90 \\
2.50\end{array}$ & 2.80 & . \\
\hline $\begin{array}{l}.043 \\
.087 \\
.130 \\
.174 \\
.261 \\
.340 \\
.439 \\
523 \\
618 \\
798 \\
798\end{array}$ & & & & & & & & & & & & & & \\
\hline & 3 & & & & & & & & & & & & & \\
\hline 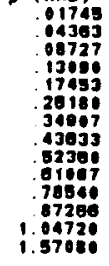 & : & 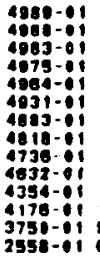 & 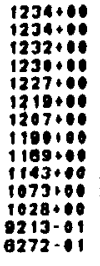 & $\begin{array}{l}2 \\
23 \\
23 \\
23 \\
22 \\
21 \\
20 \\
18 \\
17\end{array}$ & 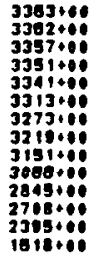 & 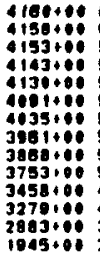 & & & & & & & & \\
\hline & - & & & & & & & & & & & & & so \\
\hline 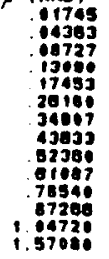 & 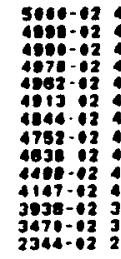 & : & ! & & & $\begin{array}{l}4 \\
4 \\
3 \\
3 \\
3 \\
3 \\
3 \\
3 \\
3\end{array}$ & & & $08+012$ & & & & แ4 2 & \\
\hline
\end{tabular}

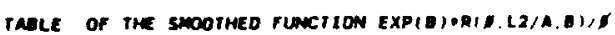

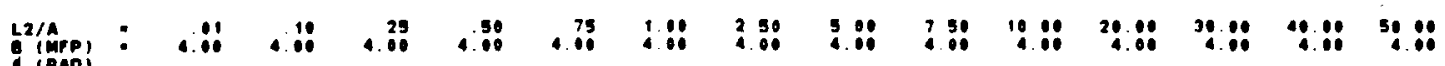

1 (AAD)

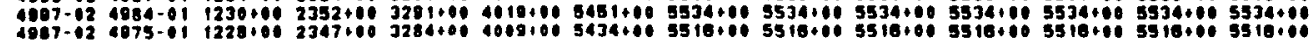

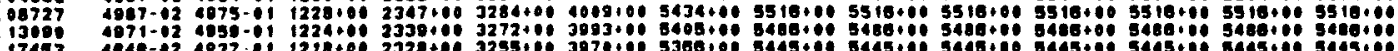

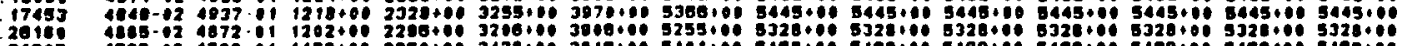

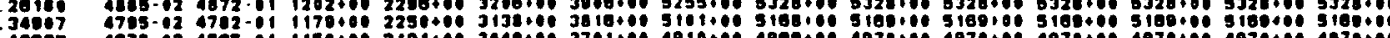

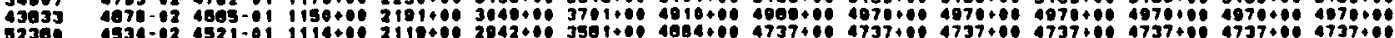

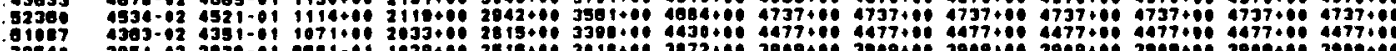

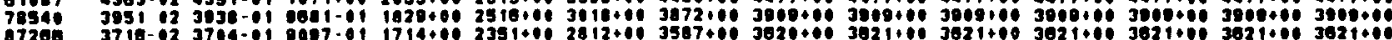

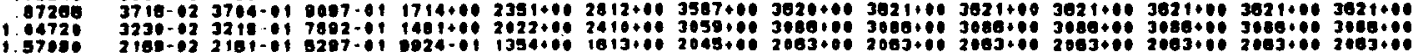

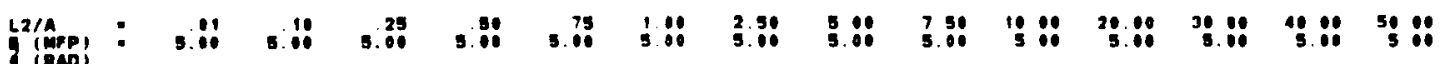

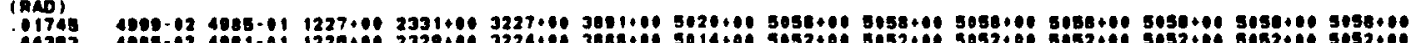

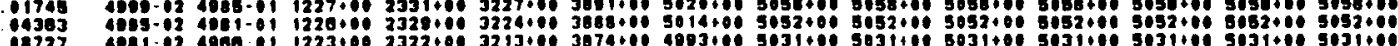

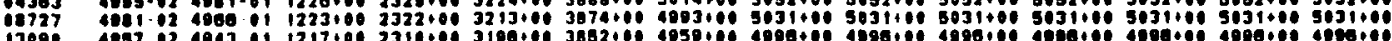

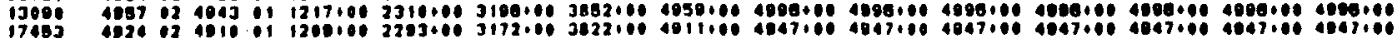

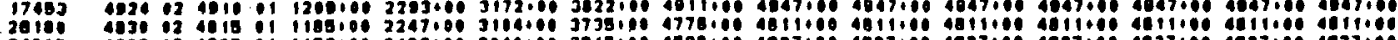

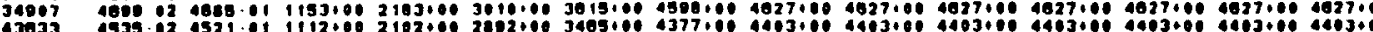

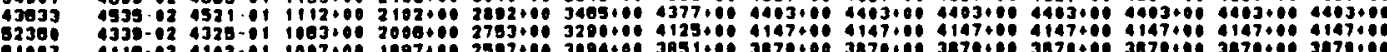

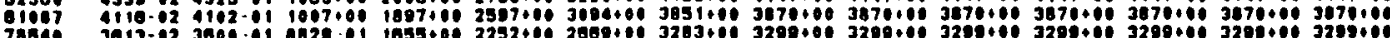

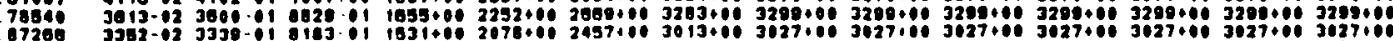

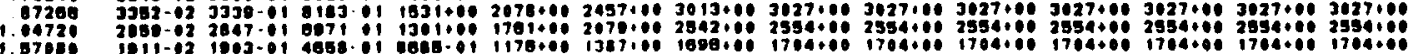

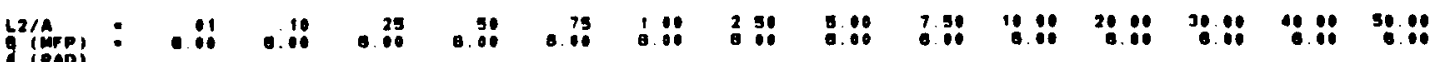

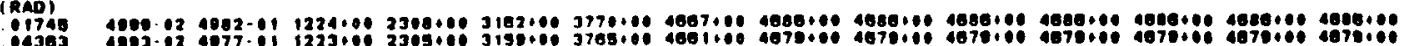

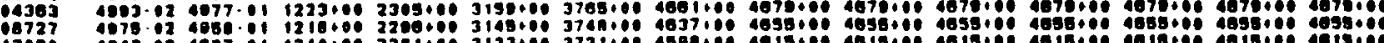

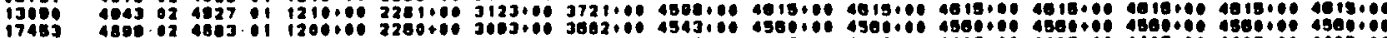

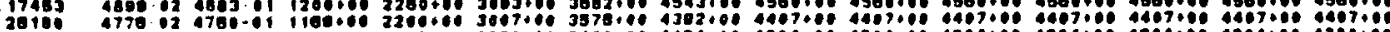

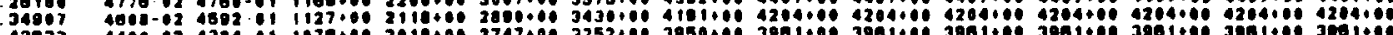

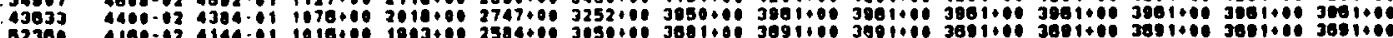

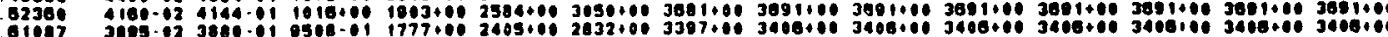

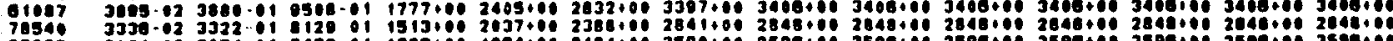

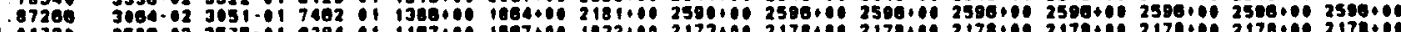

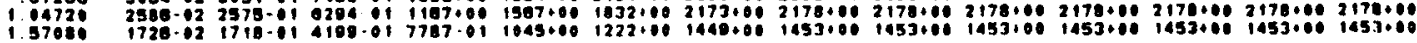


IABLE 13 (Cont'd)

TAELE OF THE SMOOTHEO FUNCTION EXPIBI-RID.L2/H.BI/,

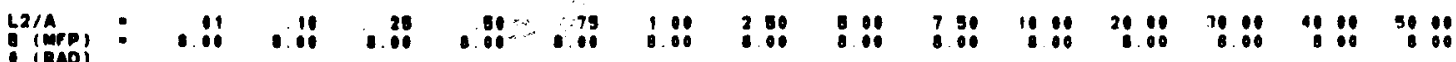

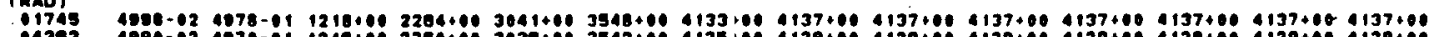

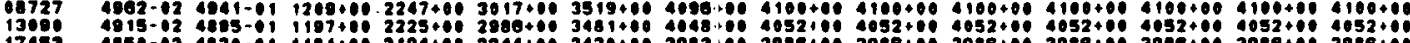

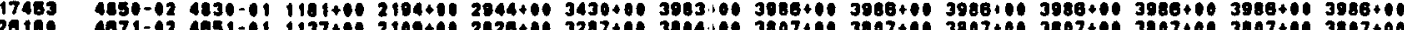

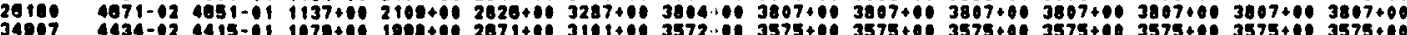

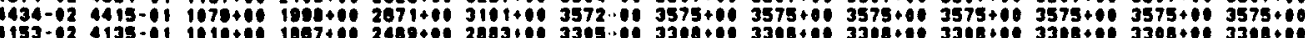

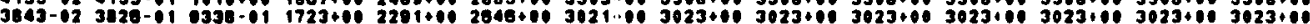

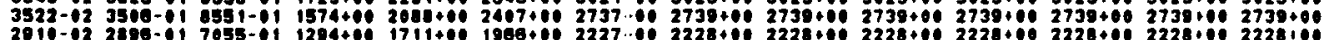

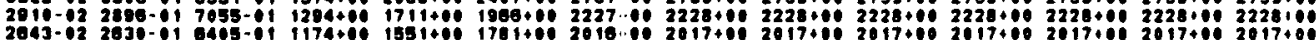

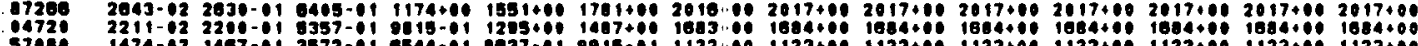

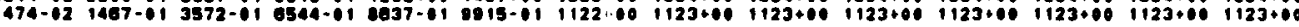

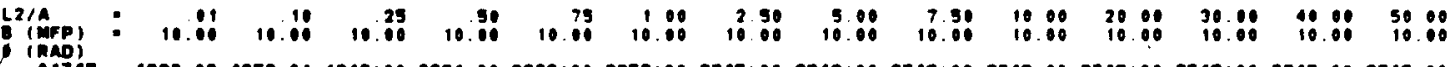

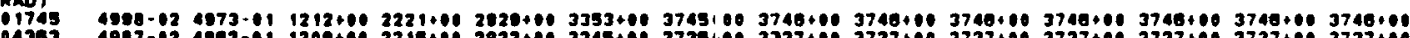

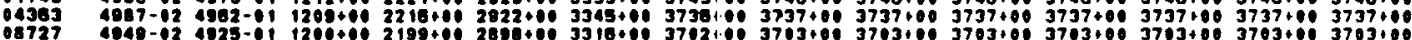

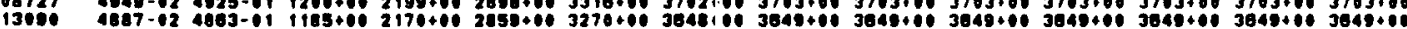

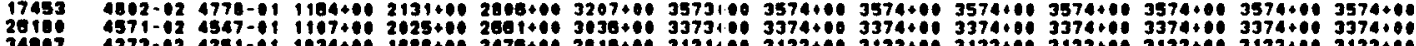
34007

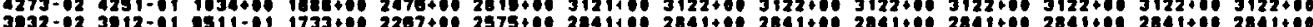

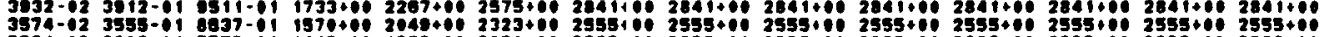

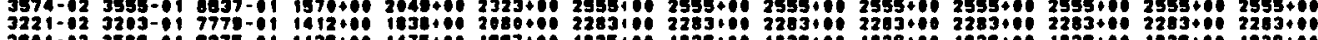

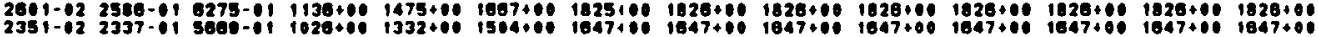

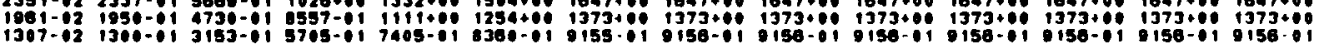

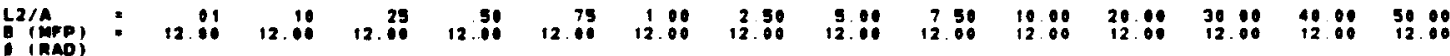

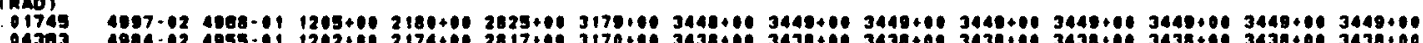

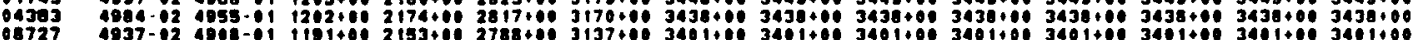

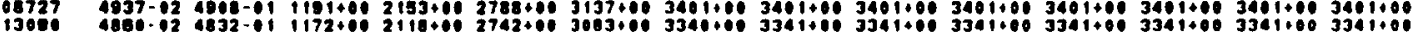

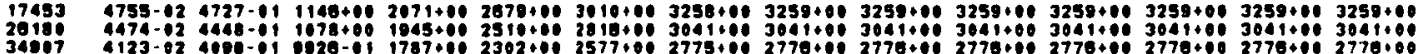

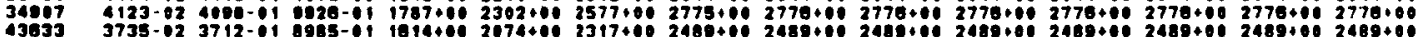

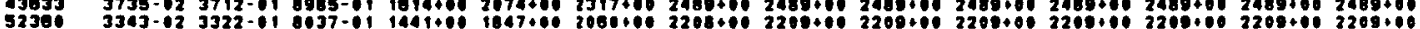

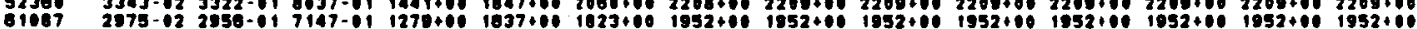

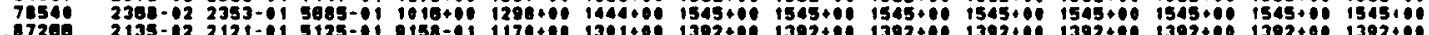

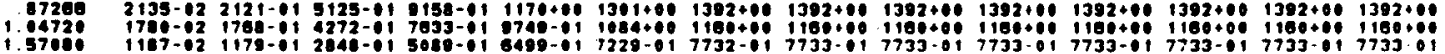

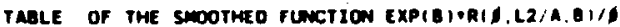

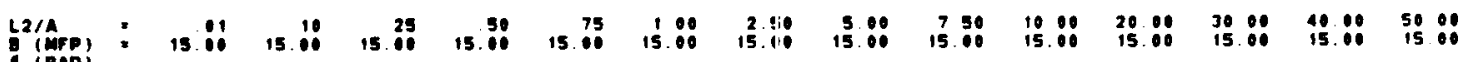

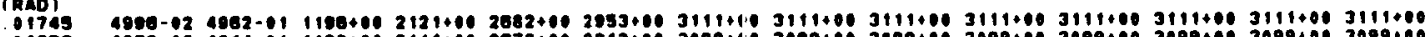

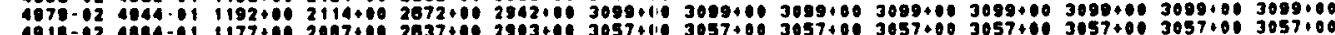

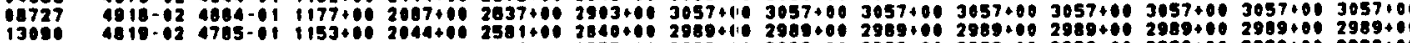

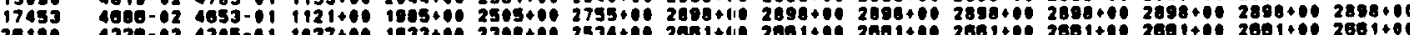

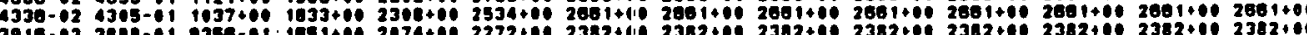

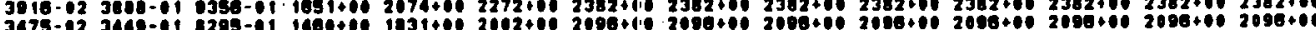

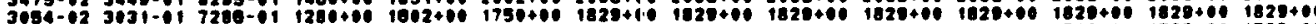

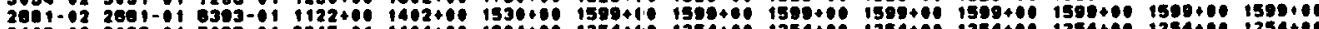

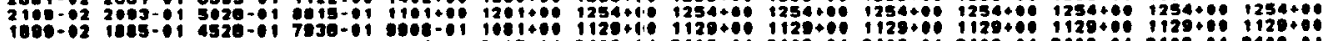

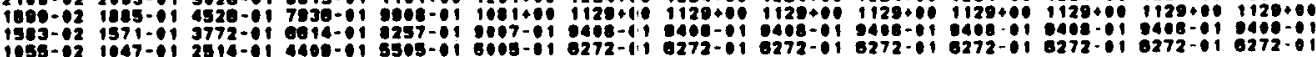

1.

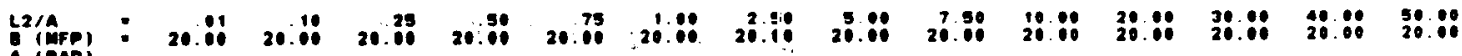

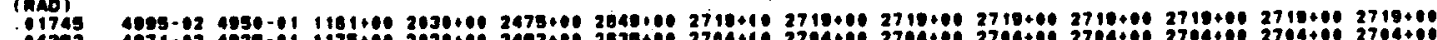



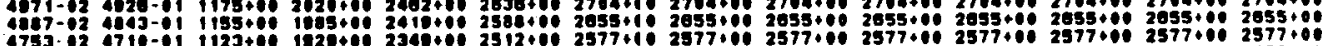

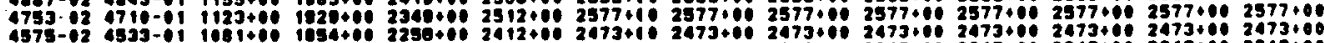

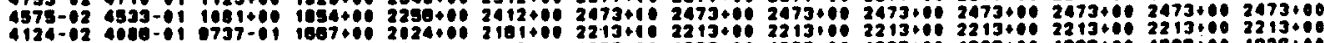

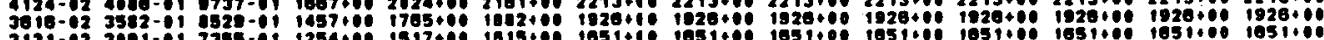

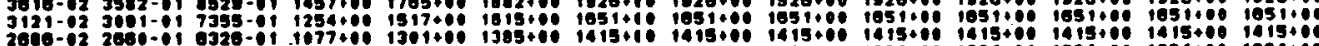

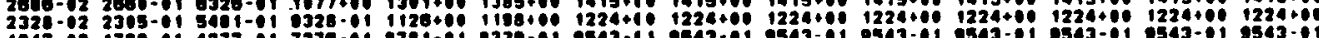

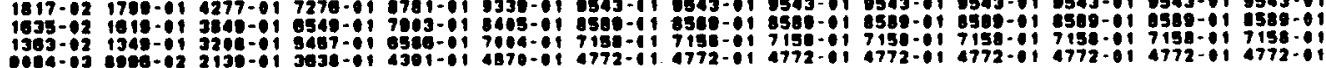

1. 8728

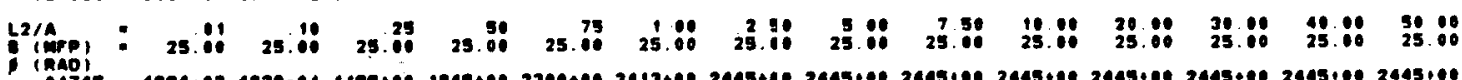

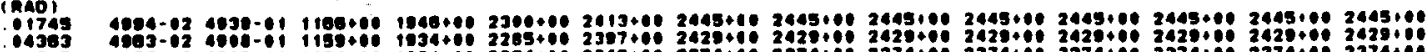

0.73

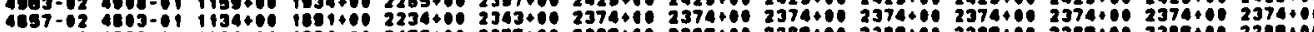

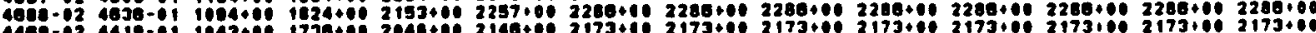

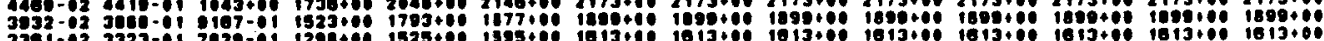

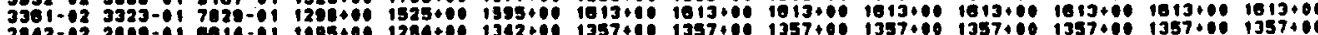

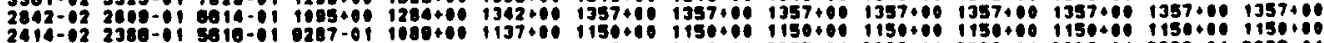

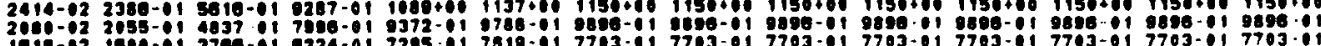

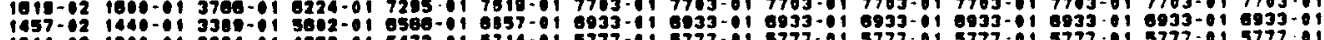

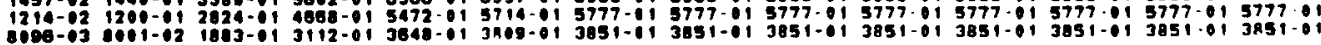


WAPD-TM-1623

\section{TABLE 13 (Cont'd)}

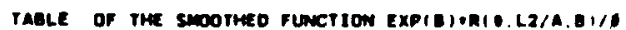

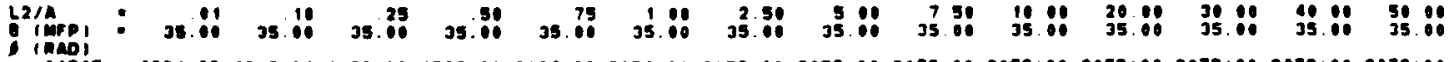

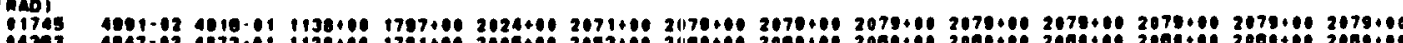

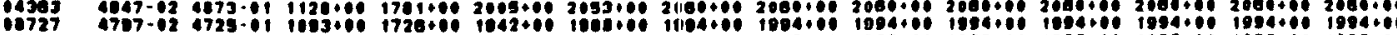

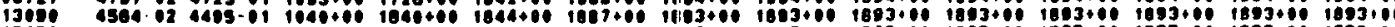

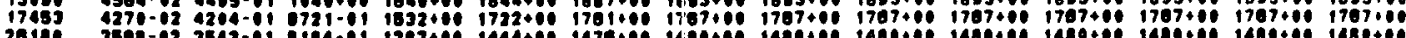

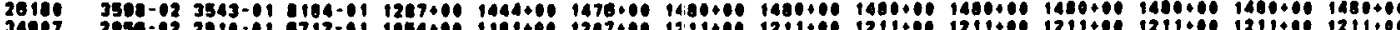

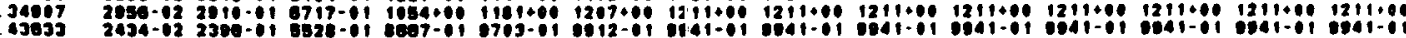
32300 2042-02 230

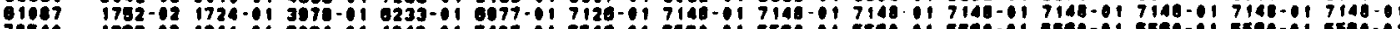

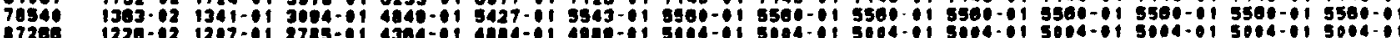

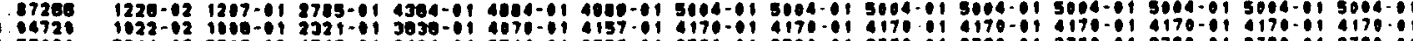

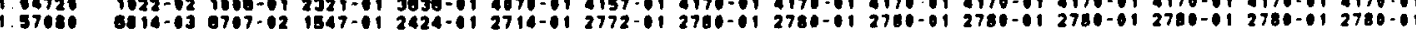

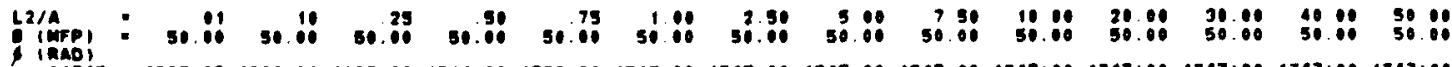

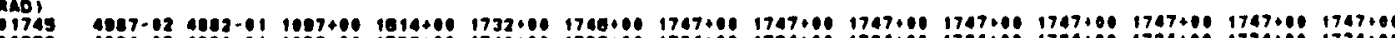

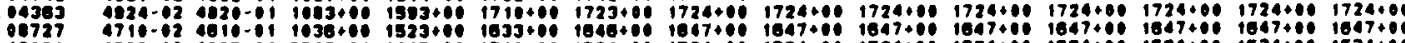

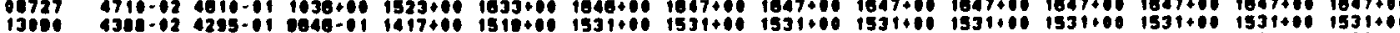

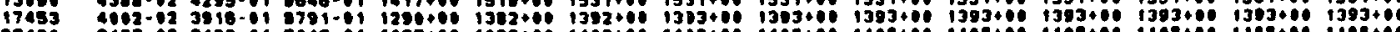

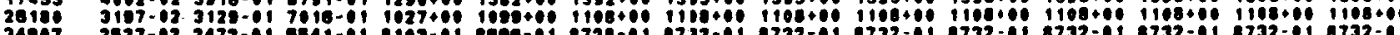

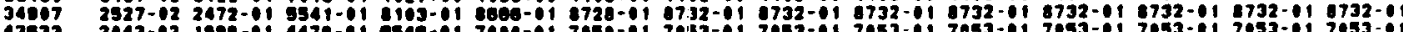

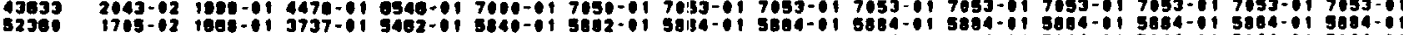

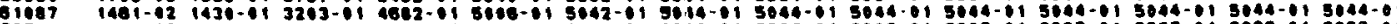

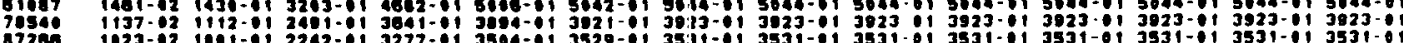

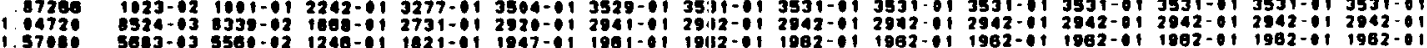

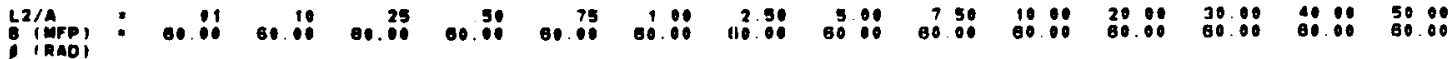
The 01745

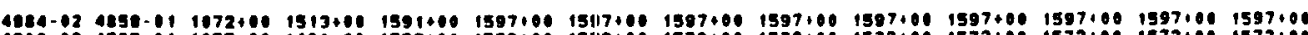

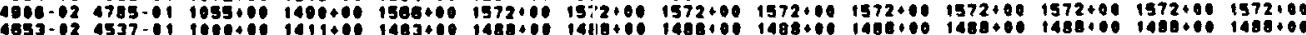

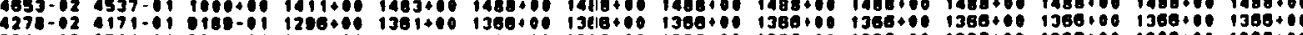

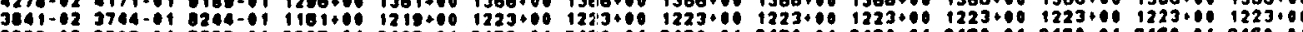

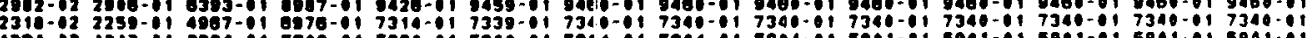

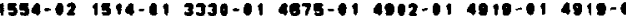

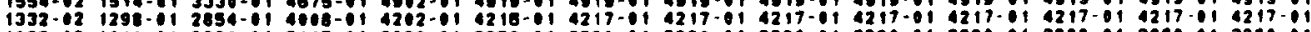
$1030-02$ 1010-01 2220-01 3117-01 3208-01 3278-01 3280--11 3280-01 3280-91 3280-01 3201-01 3286-01 3280-01 3280-91

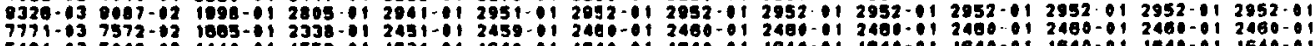

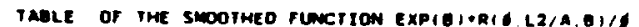

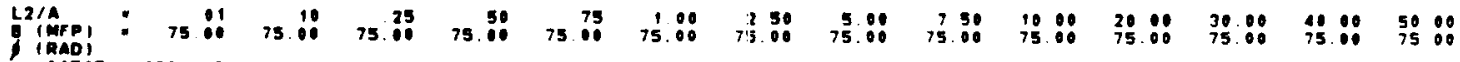

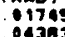

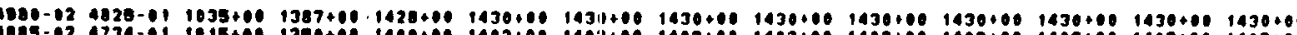

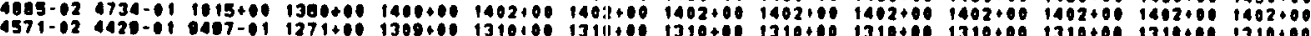

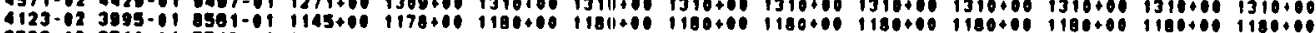

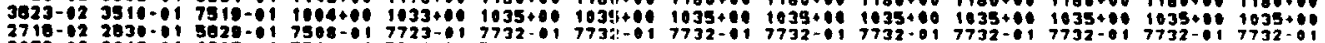

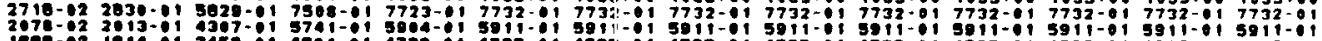

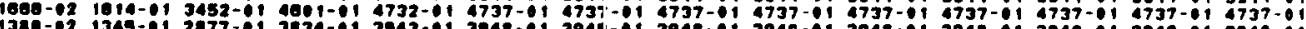

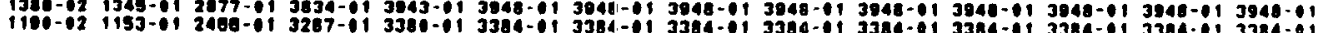

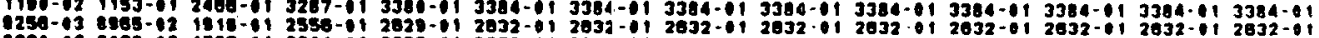

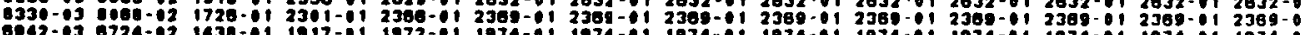

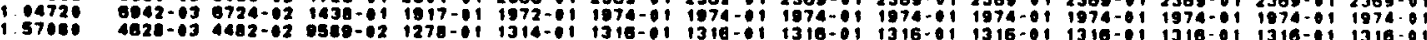


TABLE 14

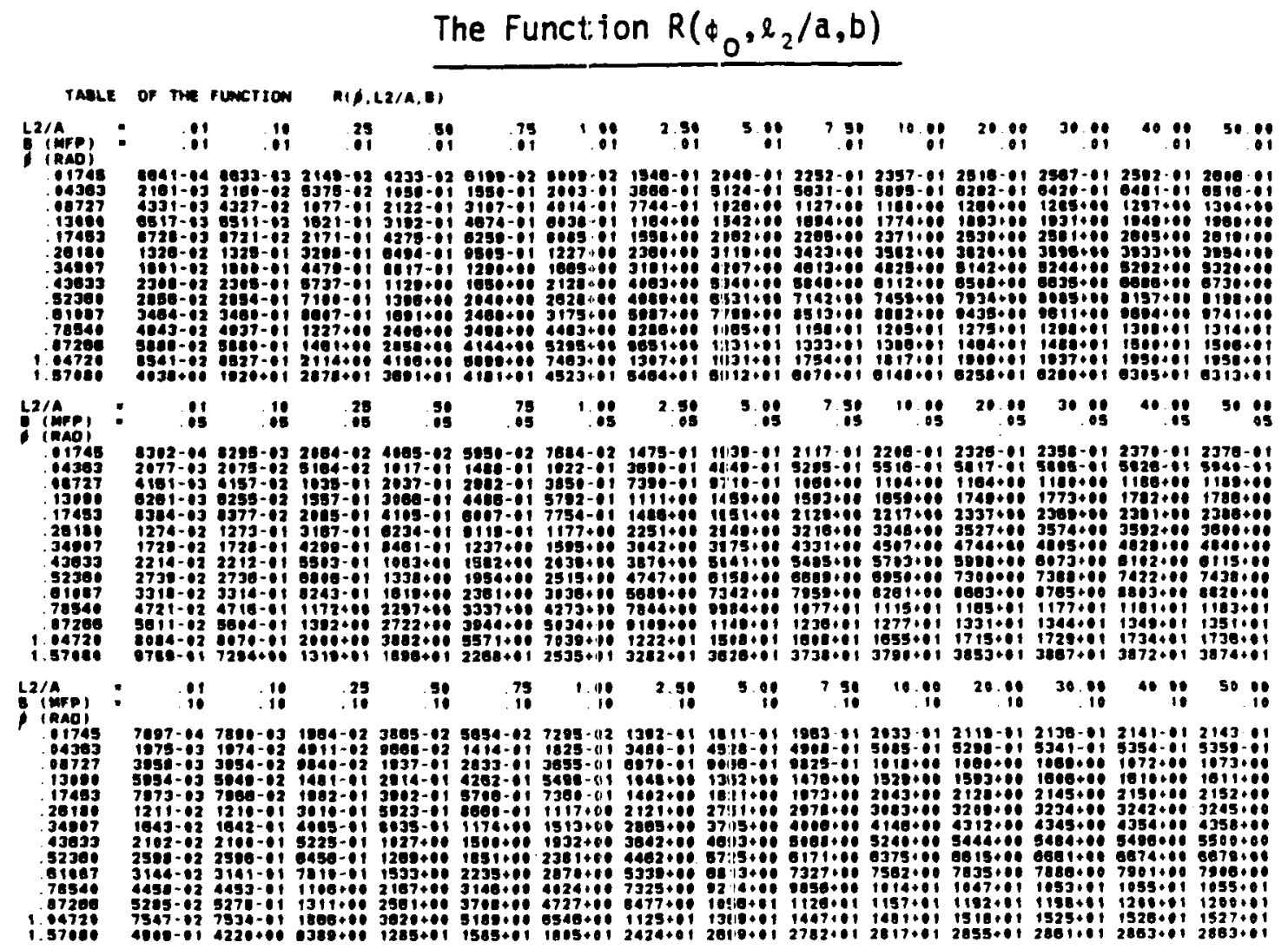

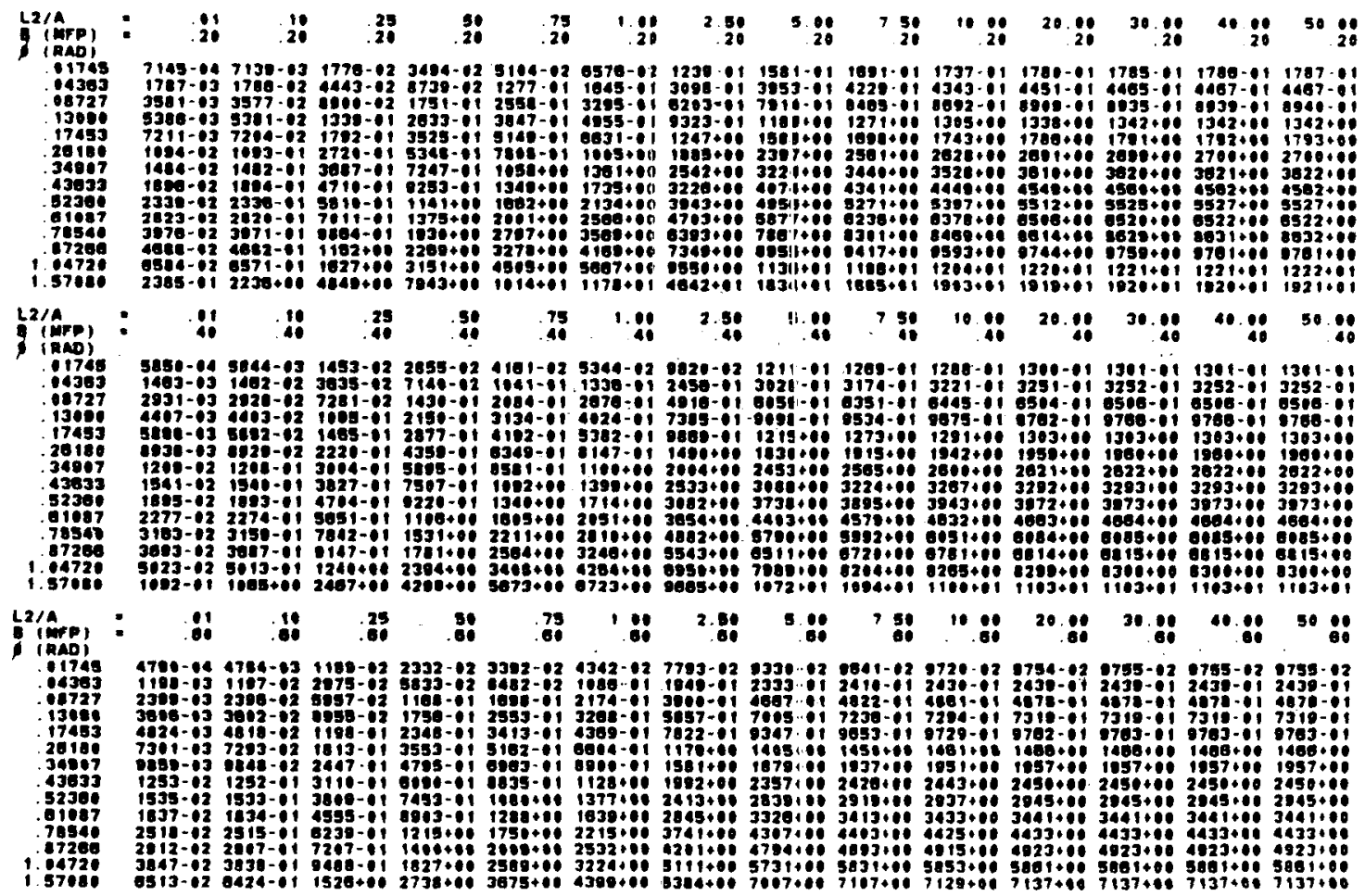


TABLE 14 (Cont'd)

\begin{tabular}{|c|c|c|c|c|c|c|c|c|c|c|c|c|c|c|}
\hline \multirow{3}{*}{ 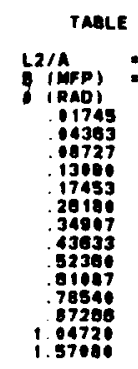 } & \multicolumn{2}{|c|}{ OF THE TUNCT1O } & \multicolumn{2}{|c|}{$R(A, L 2 / A, B)$} & \multirow[b]{2}{*}{.85} & \multirow[b]{2}{*}{1.89} & \multirow[b]{2}{*}{2.58} & \multirow[b]{2}{*}{5.80} & \multirow[b]{2}{*}{$\begin{array}{r}7 \text { s0 } \\
780\end{array}$} & \multirow[b]{2}{*}{10.88} & \multirow[b]{2}{*}{20.80} & \multirow[b]{2}{*}{ 30.80 } & \multirow[b]{2}{*}{40.81} & \multirow[b]{2}{*}{50.80} \\
\hline & $: 81$ & :18: & .85 & & & & & & & & & & & \\
\hline & $\begin{array}{r}2300 \\
2950.62 \\
4308-62\end{array}$ & 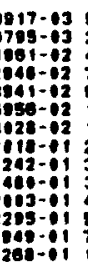 & & 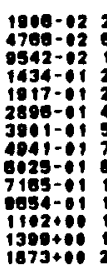 & & & & & & & & & & \\
\hline & & & $\therefore 25$ & .:B: & 1.73 & $1: 8:$ & 2.80 & $5: 08$ & 7.50 & 19.00 & 20.00 & 38.00 & $9: 80$ & $50: 00$ \\
\hline 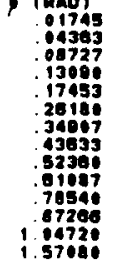 & 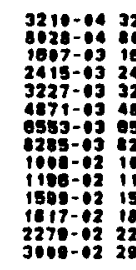 & & 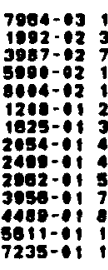 & $\begin{array}{l}11 \\
15 \\
23 \\
31 \\
80 \\
47 \\
79 \\
78 \\
18 \\
13\end{array}$ & & & & & & & $\begin{array}{l}38 \\
28 \\
57 \\
85 \\
11 \\
16 \\
19 \\
28\end{array}$ & & & 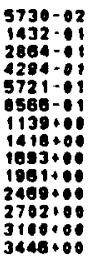 \\
\hline & $: \quad 1.25$ & 1.23 & 1.25 & $\begin{array}{r}50 \\
1.25\end{array}$ & $\begin{array}{r}75 \\
1.25\end{array}$ & 1.23 & 1.25 & $\begin{array}{l}5.00 \\
1.25\end{array}$ & $\begin{array}{l}7.30 \\
1.25\end{array}$ & 1.25 & 20.00 & $\begin{array}{l}30 \\
1.00\end{array}$ & 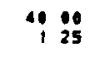 & $i$ \\
\hline 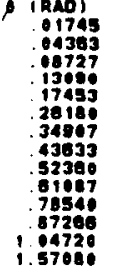 & $\begin{array}{l}2500-04 \\
0252-6 \\
1251-63 \\
1970-63 \\
2510-03 \\
3782-63\end{array}$ & & & 12 & & & & & & & & & & $\begin{array}{c}00 \\
\because \because 0 \\
00 \\
00\end{array}$ \\
\hline
\end{tabular}

TAGLE OF THE FUnCTION RIS.L.2/A.B)

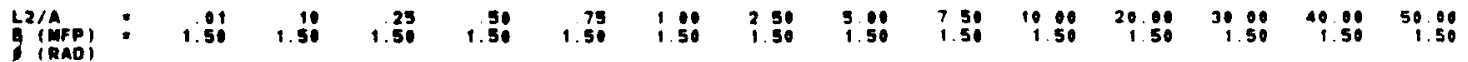

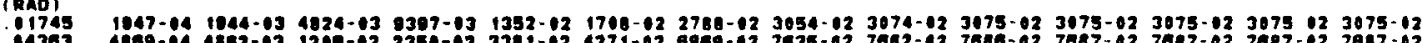

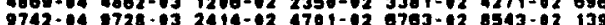

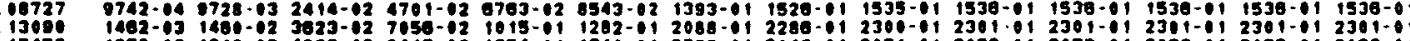

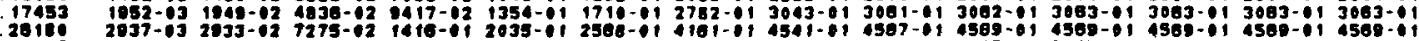

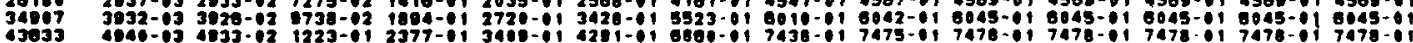

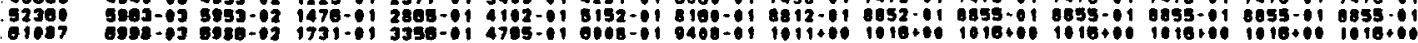

7354 904-63

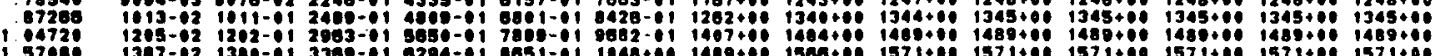

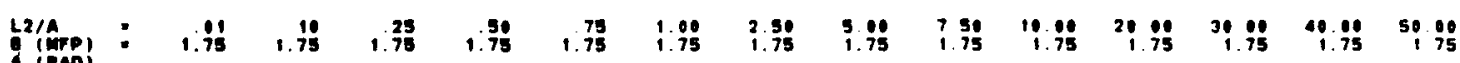

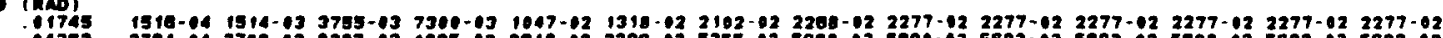

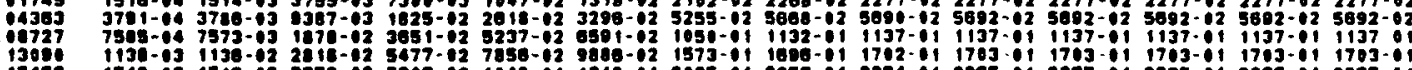

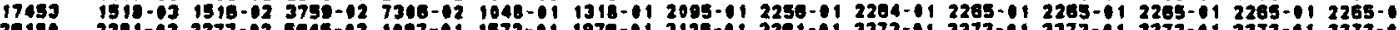

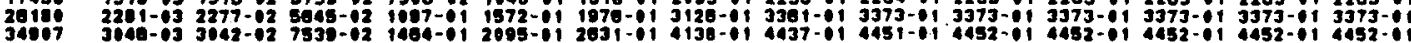

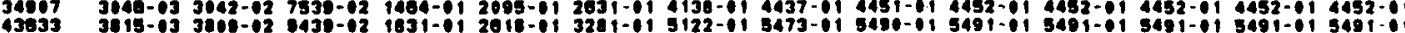

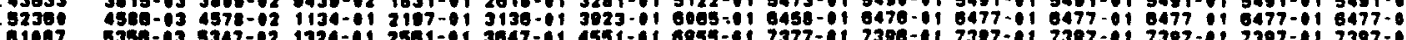

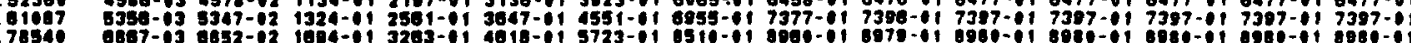

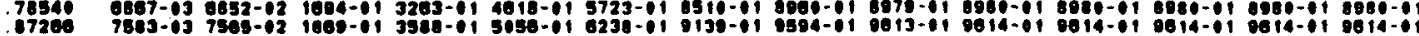

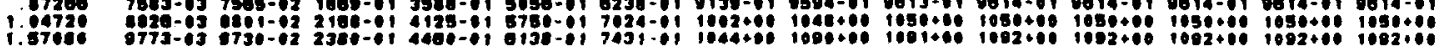

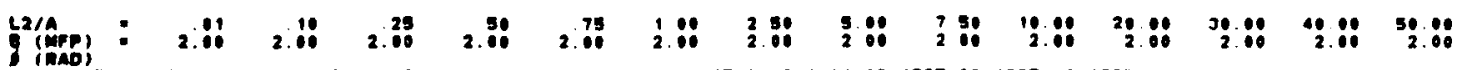

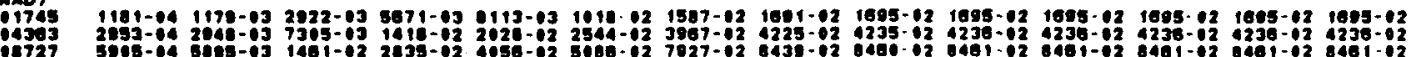

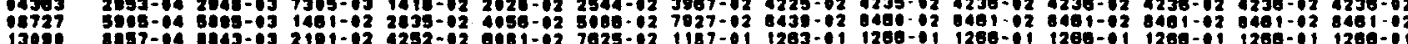

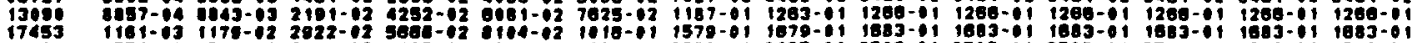

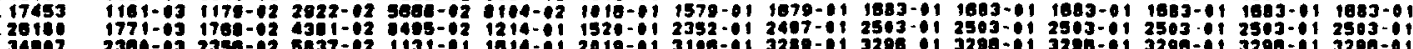

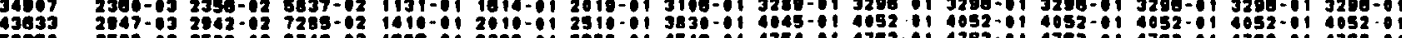

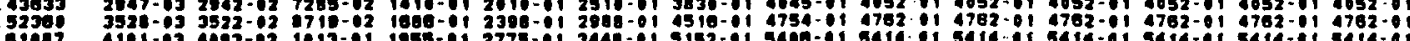

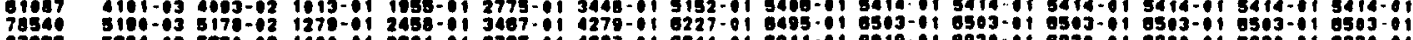

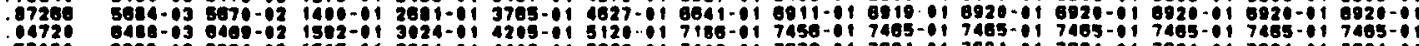

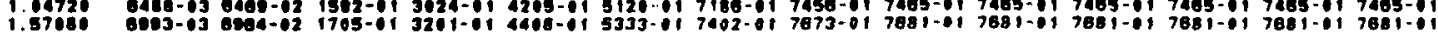


TABLE 14 (Cont'd)

\begin{tabular}{|c|c|c|c|c|c|c|c|c|c|c|c|c|c|c|}
\hline & & & & & & & & & & & & & & \\
\hline 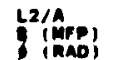 & 2.80 & 2.80 & 235 & 2.50 & 2.5 & 2.50 & 2.5 & 2.5 & & $2: 30$ & 2. & 31.90 & 40.90 & se: $: 34$ \\
\hline 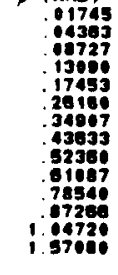 & 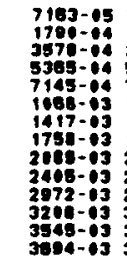 & $\begin{array}{l}71 \\
14 \\
17 \\
20\end{array}$ & $\begin{array}{l}17 \\
38 \\
34 \\
3 \\
31 \\
99 \\
73 \\
9\end{array}$ & $\begin{array}{l}36 \\
91 \\
13 \\
139 \\
19\end{array}$ & 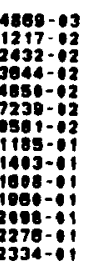 & & & $\begin{array}{l}0 \\
0 \\
0 \\
0 \\
01 \\
01 \\
01 \\
1 \\
1\end{array}$ & $\begin{array}{l}940 . \\
9394 \\
1020 \\
2234 \\
2008 \\
2938 \\
3408\end{array}$ & 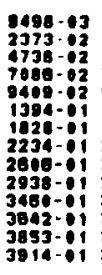 & 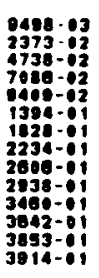 & 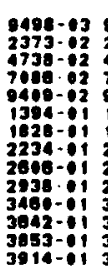 & 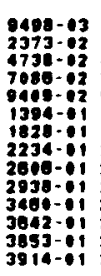 & 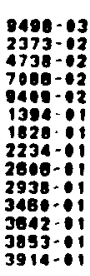 \\
\hline & $3: 1$ & $3: 10$ & 3.25 & 3. & 3 & $3: 0$ & $\begin{array}{lll}2 & 50 \\
3 & 90\end{array}$ & 3.:0 & 3.5 & $\begin{array}{r}10.00 \\
3.00\end{array}$ & 20.00 & 30.08 & $40: 0$ & ${ }_{3}^{50}: 8$ \\
\hline 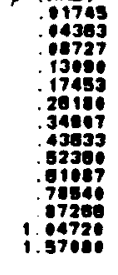 & 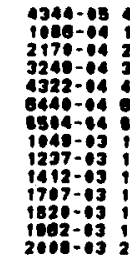 & & & & 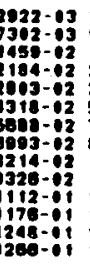 & & & & & 3 & $\begin{array}{l}80 \\
43 \\
73 \\
10 \\
12 \\
18 \\
18 \\
18 \\
10\end{array}$ & s. & 190 & 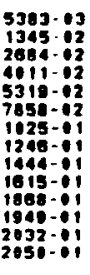 \\
\hline & 330 & 3. & 3.58 & 3.50 & 3.5 & 'ं. & $\begin{array}{l}2.5 \\
3.5\end{array}$ & $\begin{array}{l}5.0 \\
3.5\end{array}$ & 3 & 10.8 & 30 & 30. & 3.50 & 50 \\
\hline 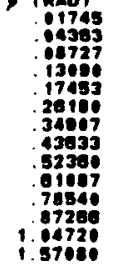 & 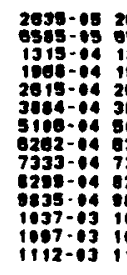 & & . & & & & & & & & & 12 & & \\
\hline
\end{tabular}

TAOLE OF THE FUNCTION R(G,L2/A.Q1

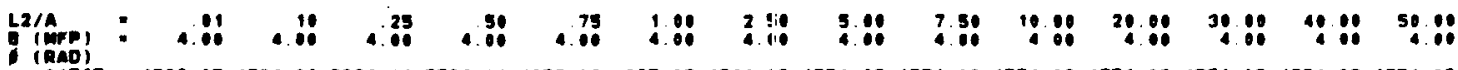

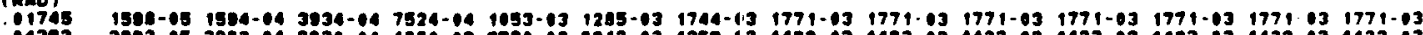

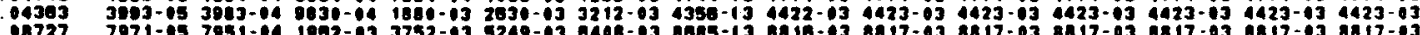

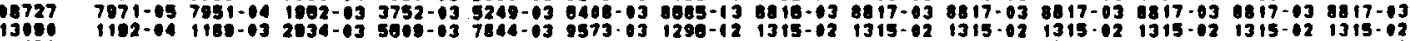

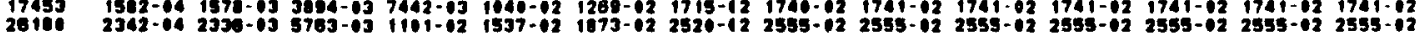

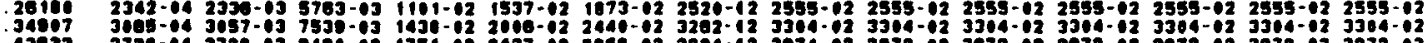

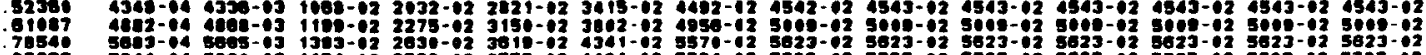

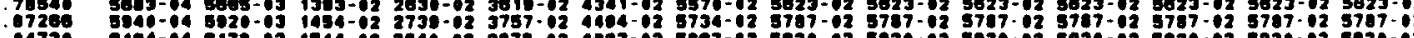

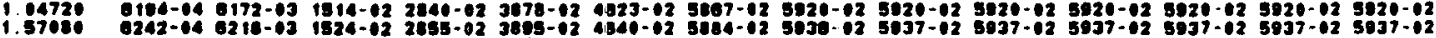

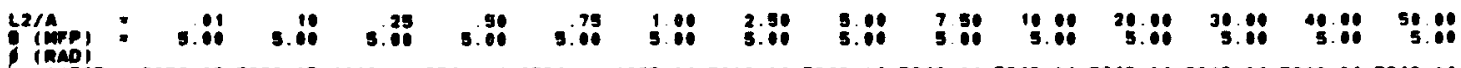

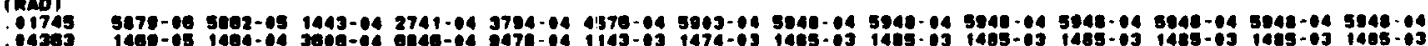

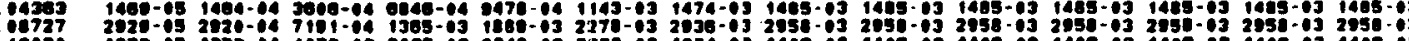

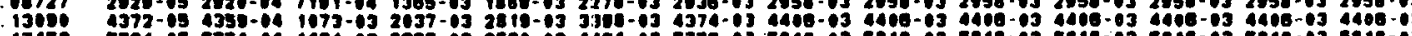

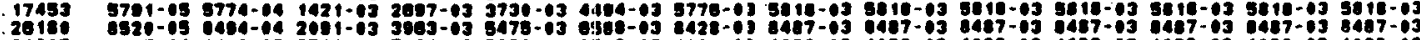
.34967

23

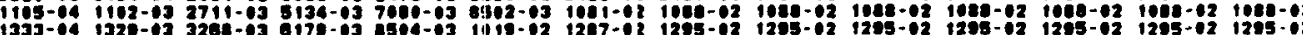

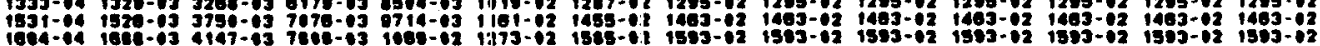

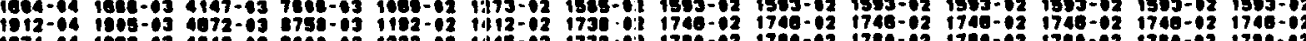

a7200

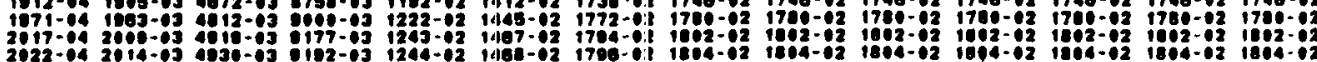

L2/A

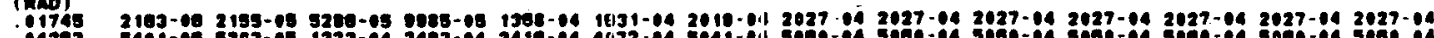
0.903

:47373

13909

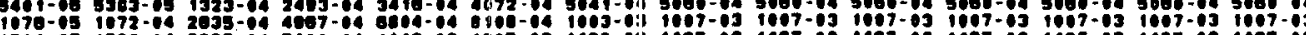

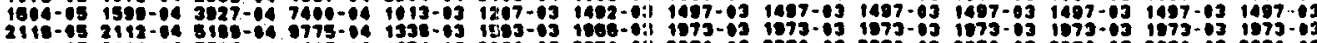

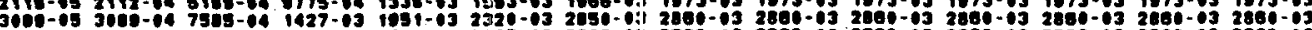

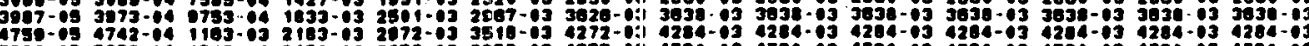

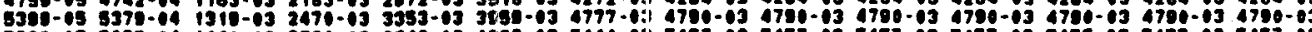

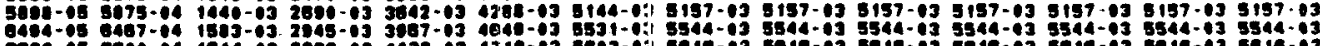

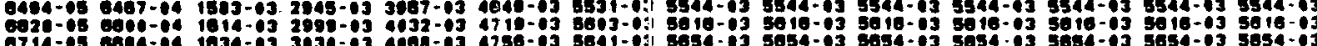

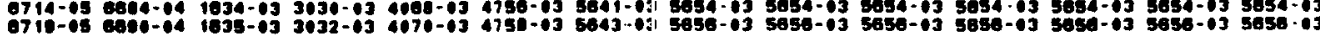


WAPD-TM-1623

TABLE 14 (Cont'd)

\begin{tabular}{|c|c|c|c|c|c|c|c|c|c|c|c|c|c|c|}
\hline 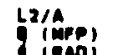 & $: \quad \because 1$ & ! & .23 & .80 & $\begin{array}{r}75 \\
0.06\end{array}$ & $1: 0$ & $25:$ & 50 & $7.5:$ & 1980 & $28: 06$ & 30.01 & 40.10 & 50.00 \\
\hline 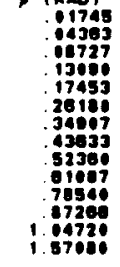 & 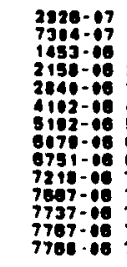 & 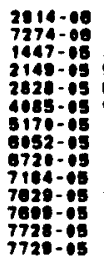 & 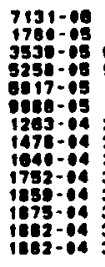 & 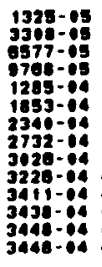 & 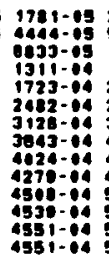 & 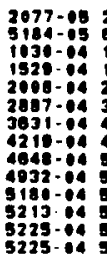 & 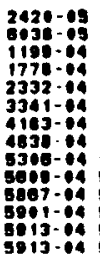 & 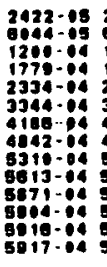 & 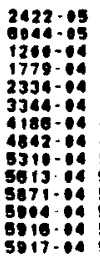 & $\begin{array}{l}-04 \\
-84 \\
-84 \\
-84 \\
-04 \\
-14 \\
-14\end{array}$ & $\begin{array}{l}212 \\
604 \\
120 \\
177 \\
233 \\
334 \\
118 \\
484 \\
831 \\
691 \\
507 \\
590 \\
591 \\
591\end{array}$ & 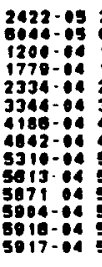 & & 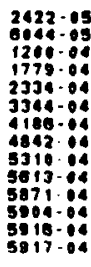 \\
\hline (map) & $: 10.01$ & 10.10 & 10.29 & 10.80 & 10.75 & 10.86 & $\begin{array}{r}2.50 \\
10.10\end{array}$ & 10.06 & $10: 9$ & 10.10 & & $\begin{array}{l}30.10 \\
10.00\end{array}$ & $10 \%$ & $\begin{array}{l}50 \% 0 \\
10 \% 0\end{array}$ \\
\hline 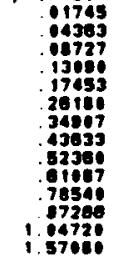 & $\begin{array}{l}3960-1 \\
9379-1 \\
1961-0 \\
2904-8 \\
3905-1 \\
8432-1 \\
6771-0 \\
7709-9\end{array}$ & 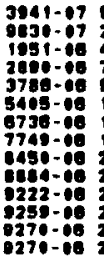 & 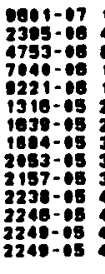 & $\begin{array}{l}1 \\
1 \\
1 \\
2 \\
2 \\
3\end{array}$ & 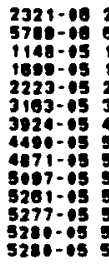 & & & & & $\begin{array}{l}2998-00 \\
7492-09 \\
1467-05 \\
2108-05 \\
2932-65\end{array}$ & & $\begin{array}{l}-06 \\
-00 \\
-05 \\
-05 \\
-05 \\
-05 \\
-45 \\
05\end{array}$ & $\begin{array}{l}-00 \\
2.06 \\
7.05 \\
-05 \\
-05 \\
-05 \\
-05 \\
-05\end{array}$ & \\
\hline & $: 12: 1$ & $12: 10$ & 12.25 & $12 \%$ & $12 ?$ & $12: 0$ & $\begin{array}{r}2.50 \\
12.00\end{array}$ & $12: 0$ & $\begin{array}{r}750 \\
1200\end{array}$ & $\begin{array}{l}10 \% \\
12: 0\end{array}$ & & $\begin{array}{ll}30 & 00 \\
12 & 06\end{array}$ & $12: 10$ & $\begin{array}{ll}50 & \because 2 \\
12 & 00\end{array}$ \\
\hline 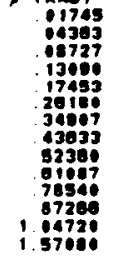 & 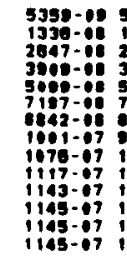 & 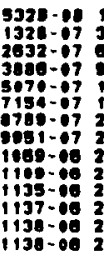 & 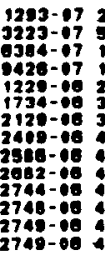 & 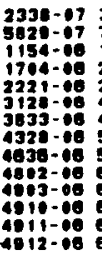 & 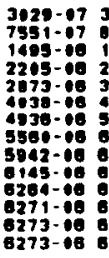 & 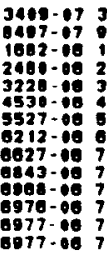 & $\begin{array}{l}7-17 \\
3-06 \\
7-06 \\
4-80 \\
2-80 \\
2-08 \\
3-96\end{array}$ & $\begin{array}{ll}7327-06 \\
7454-08 \\
7482-08 \\
7483-08 \\
7463-06\end{array}$ & 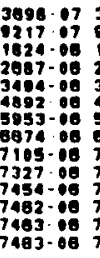 & $7463-067$ & 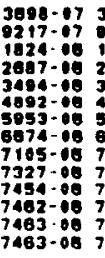 & 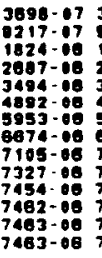 & $\begin{array}{l}7327-06 \\
7454-60 \\
7402-06 \\
7463-00 \\
7483-06\end{array}$ & $\begin{array}{l}9217-07 \\
1024-06 \\
2697-06 \\
3494-06 \\
1892-06 \\
3953-06 \\
6674-06 \\
7165-06 \\
7327-06 \\
7454-06 \\
7462-06 \\
7463.06 \\
7463-08\end{array}$ \\
\hline
\end{tabular}

TABLE of THE FUnCIION RIS,L2/A,BI

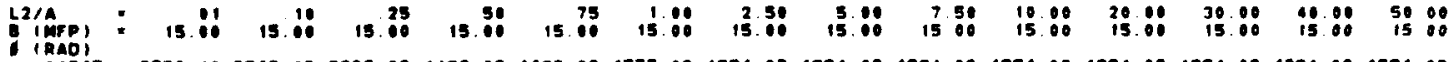

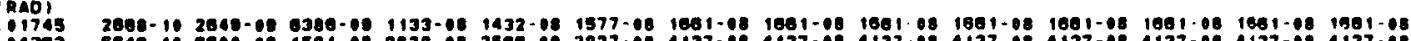

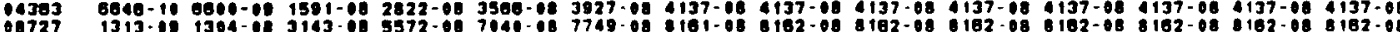

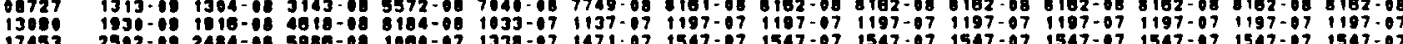

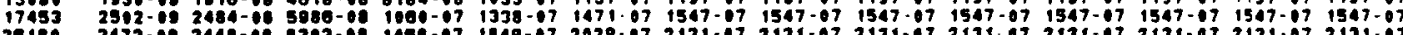

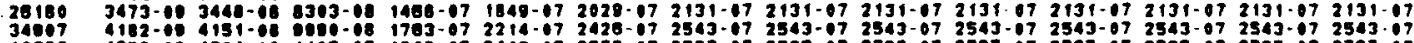

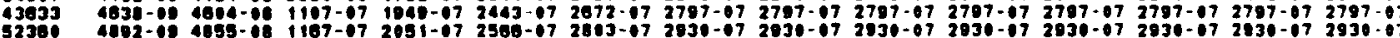

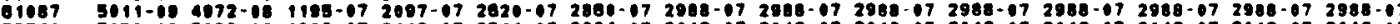

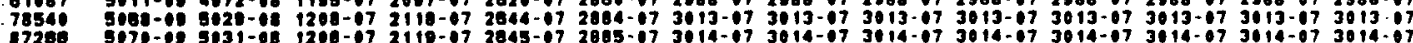

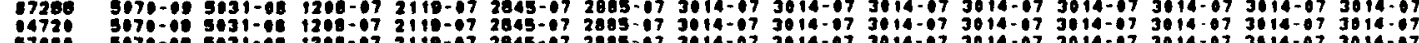

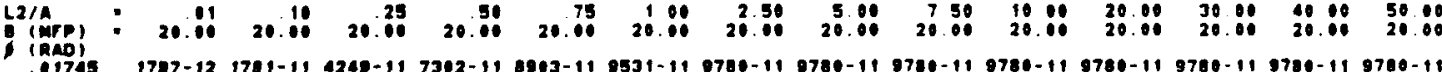

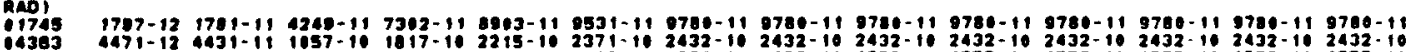

04363 4471-12 4431-1t 1057-10 1917-10 2215-10 2371-1: 2432-10 2432-10 2432-10 2432-10 2432-10 2432-10 2432-10 2432-10

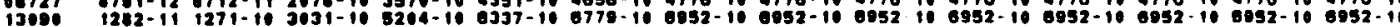

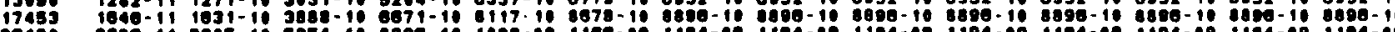

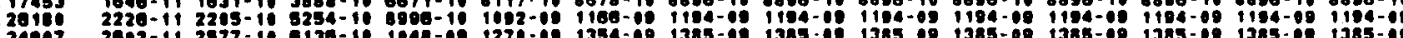

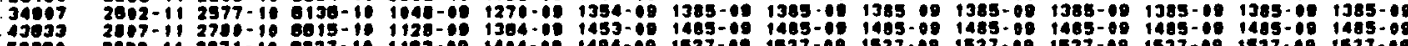

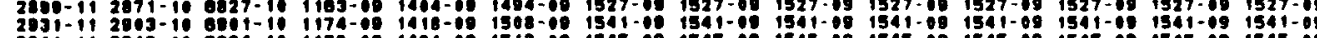

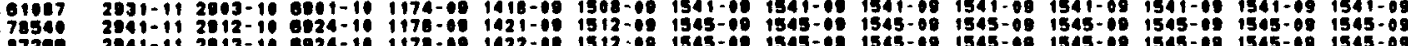

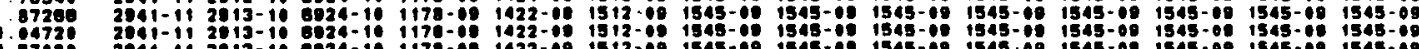

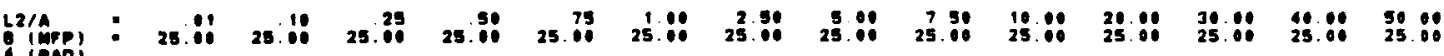

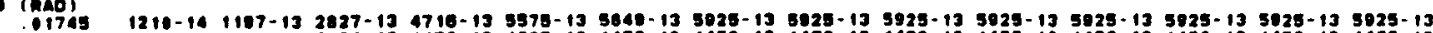

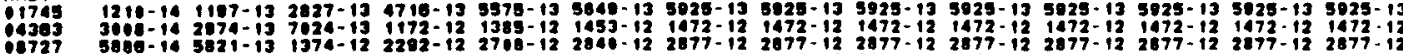

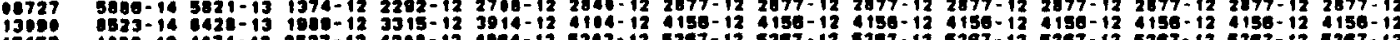

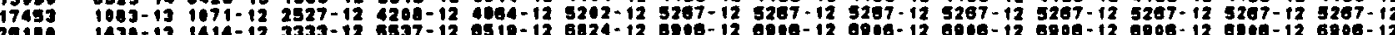

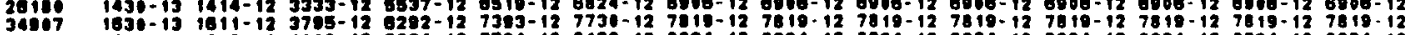

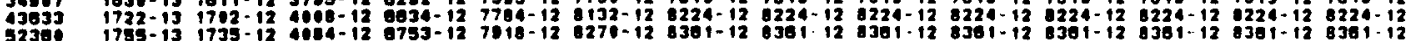

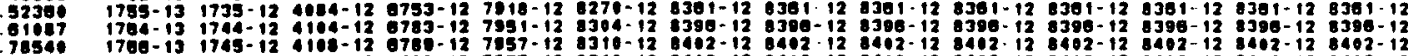

7ases

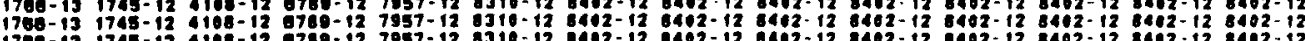

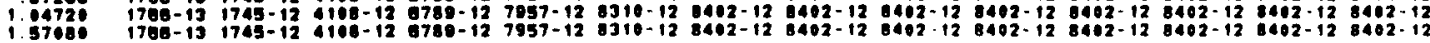




\section{TABLE 14 (Cont'd)}

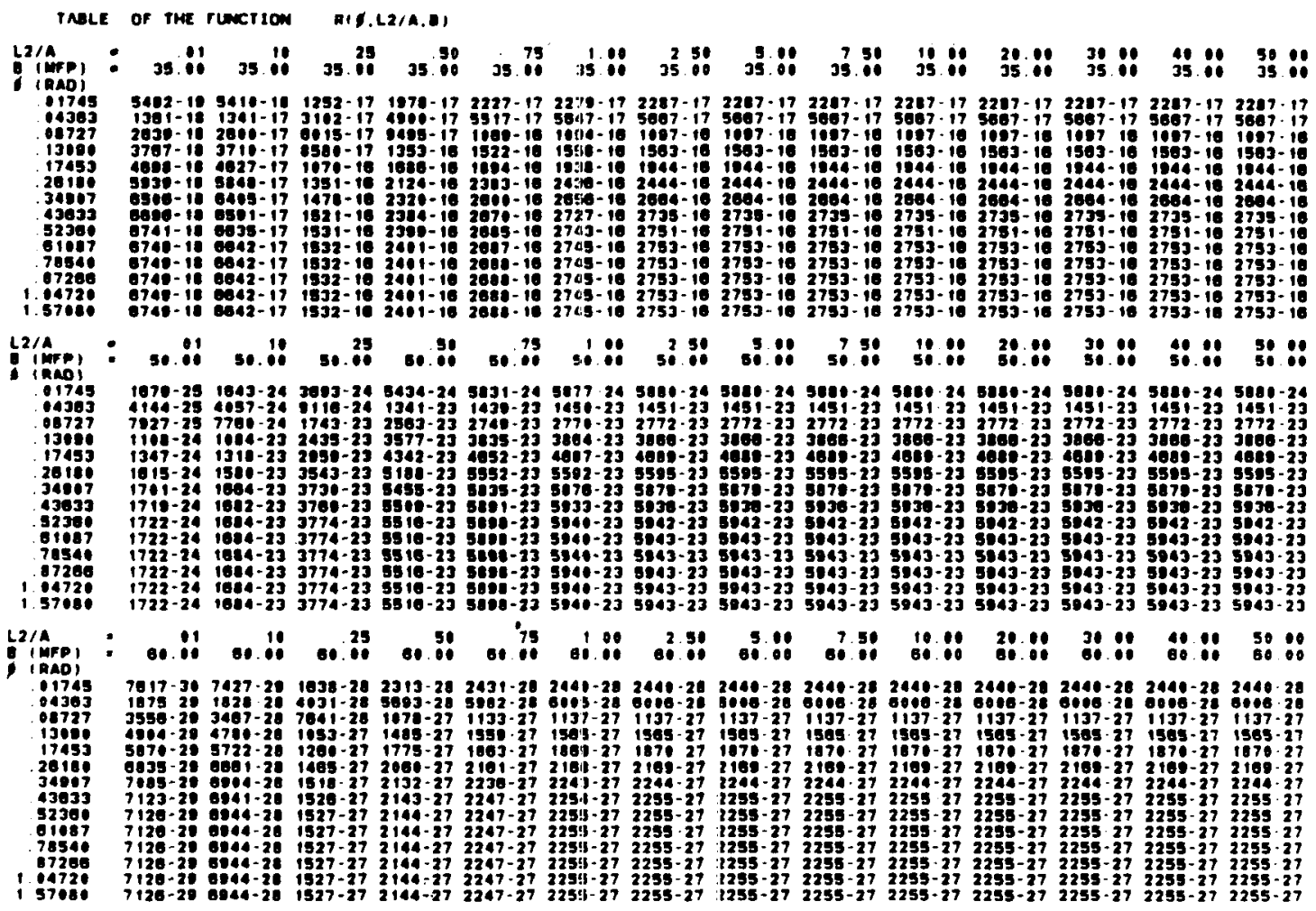

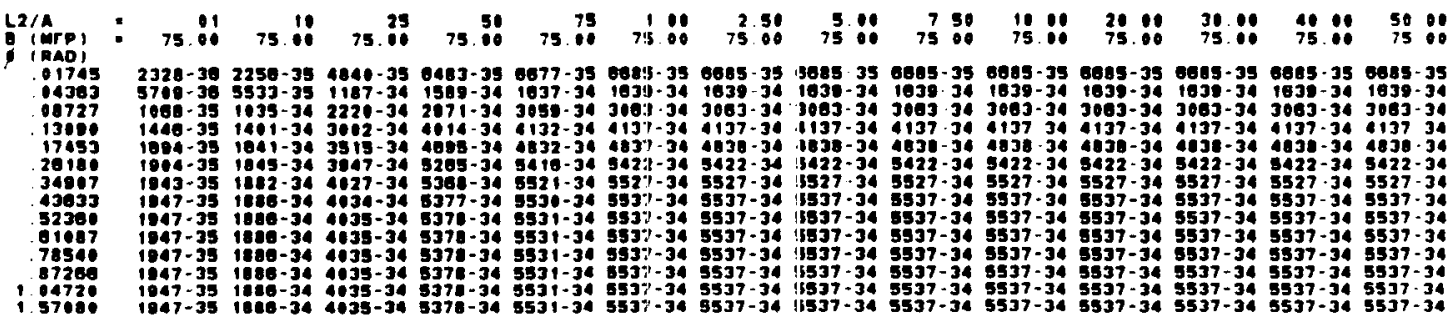


TABLE 15

The Function $E_{1}(b)$

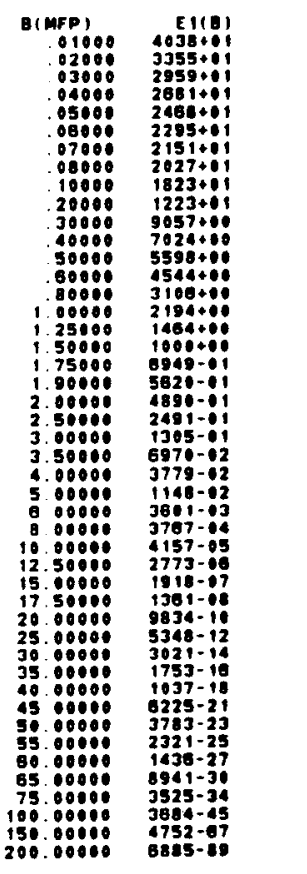

TABLE 17

The Function $E_{2}(b)$

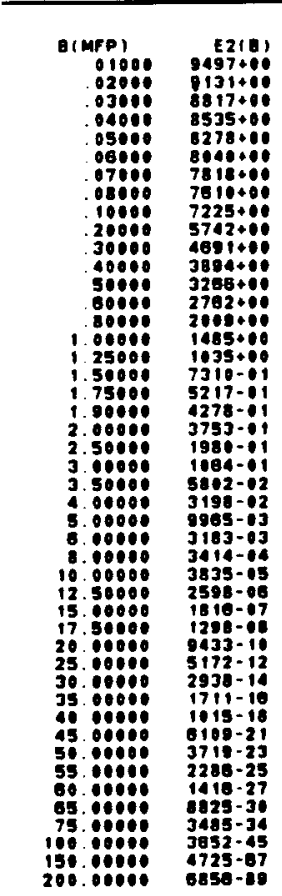

TABLE 16

The Smoothed Function

$\bar{E}_{1}(b)=b e^{b} E_{1}(b)$

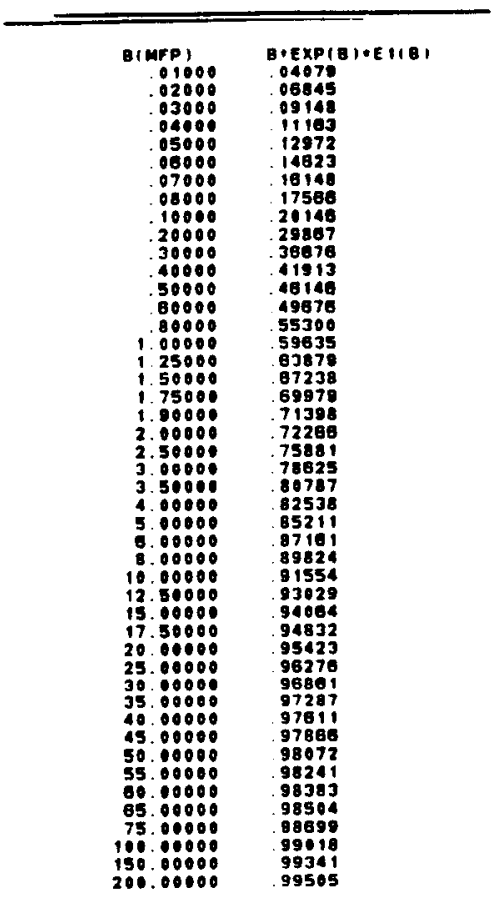

TABLE 18

The Smoothed Function $\bar{E}_{2}(b)=b e^{b} E_{2}(b)$

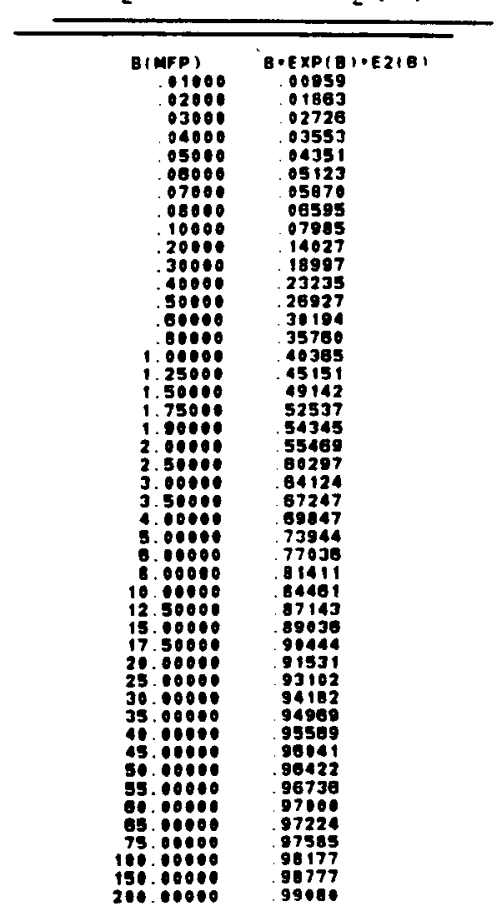




\section{References}

1. 0. J. Wallace, "Analytic Flux Formulas and Tables of Shielding Functions," WAPD-TM-1453, June 1981.

2. K. Shure and 0. J. Wallace, "Complact Tables of Functions for Use in Shielding Calculations," Nuclear Science and Engineering, 56, pp. 84-89 (1975).

3. 0. J. Wallace, "Semi-Analytic Flux Formulas for Shielding Calculations," WAPD-TM-1197, May 1976.

4. H. Ono and A. Tsuro, "An Approximate Calculation Method of Flux for Spherical and Cylindrical Sources With a Slab Shield," J. Nuc. Sci. and Tech., 2, \#6, pp. 229-235 (June 1965).

5. 0. J. Wallace, "SPAR1 - A Semi-Analytic Point-Kernel Computer Program for Shielding," WAPD-TM-1196, June 1976.

6. D. Van Nostand, "Reactor Shielding Design Manual," T. Rockwe11, Ed., Princeton, NJ (1956). 